\title{
Uncertainty Quantification of Composite Laminate Damage with the Generalized Information Theory
}
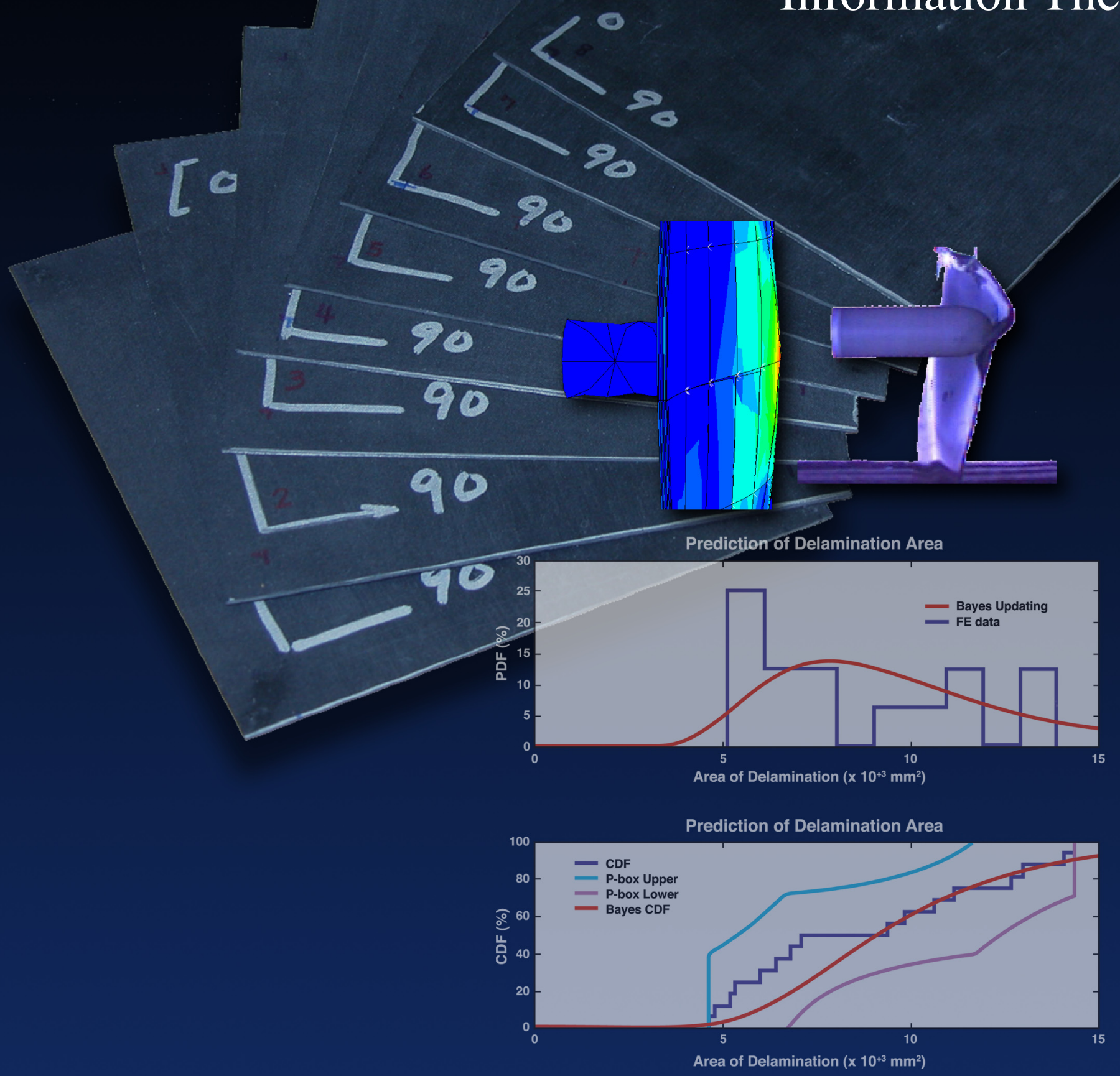


\begin{abstract}
About the cover:
The cover shows several components used in the impact-damage studies described in the report. In the background are eight of the multilayered carbon-epoxy composite plates used in the impact experiments. In front of the eight plates, at left, is a finite-element simulation at the moment of impact. At right is a snapshot from an impact experiment. In the foreground are plots of several types of data analysis.
\end{abstract}

Los Alamos National Laboratory, an affirmative action/ equal opportunity employer, is operated by the University of California for the United States Department of Energy under contract W-7405-ENG-36.

This report was prepared as an account of work sponsored by an agency of the United States Government. Neither the Regents of the University of California, the United States Government nor any agency thereof, nor any of their employees make any warranty, express or implied, or assume any legal liability or responsibility for the accuracy, completeness, or usefulness of any information, apparatus, product, or process disclosed, or represent that its use would not infringe privately owned rights. Reference herein to any specific commercial product, process, or service by trade name, trademark, manufacturer, or otherwise does not necessarily constitute or imply its endorsement, recommendation, or favoring by the Regents of the University of California, the United States Government, or any agency thereof. The views and opinions of authors expressed herein do not necessarily state or reflect those of the Regents of the University of California, the United States Government, or any agency thereof. Los Alamos National Laboratory strongly supports academic freedom and a researcher's right to publish; as an institution, however, the Laboratory does not endorse the viewpoint of a publication or guarantee its technical correctness. 
LA-14283

Issued: May 2006

\title{
Uncertainty Quantification of Composite \\ Laminate Damage with the Generalized \\ Information Theory
}

\author{
Jonathan Lucero \\ François Hemez \\ Timothy Ross* \\ Kinnan Kline \\ Jason Hundhausen \\ Trevor Tippetts
}





\title{
Uncertainty Quantification of Composite Laminate Damage With the Generalized Information Theory
}

\author{
Final Report of the FY05 ASC-V\&V-Engineering Analysis Level-5 Milestone
}

\author{
By: Jonathan Lucero ${ }^{1}$, François Hemez ${ }^{2}$, Timothy Ross ${ }^{3}$, \\ Kinnan Kline ${ }^{4}$, Jason Hundhausen ${ }^{5}$, Trevor Tippetts ${ }^{6}$ \\ Los Alamos National Laboratory, Los Alamos, New Mexico 87545
}

\begin{abstract}
This work presents a survey of five theories to assess the uncertainty of projectile impact induced damage on multi-layered carbon-epoxy composite plates. Because the types of uncertainty dealt with in this application are multiple (variability, ambiguity, and conflict) and because the data sets collected are sparse, characterizing the amount of delamination damage with probability theory alone is possible but incomplete. This motivates the exploration of methods contained within a broad Generalized Information Theory (GIT) that rely on less restrictive assumptions than probability theory. Probability, fuzzy sets, possibility, and imprecise probability (probability boxes (p-boxes) and Dempster-Shafer) are used to assess the uncertainty in composite plate damage. Furthermore, this work highlights the usefulness of each theory. The purpose of the study is not to compare directly the different GIT methods but to show that they can be deployed on a practical application and to compare the assumptions upon which these theories are based. The data sets consist of experimental measurements and finite element predictions of the amount of delamination and fiber splitting damage as multilayered composite plates are impacted by a projectile at various velocities. The physical experiments consist of using a gas gun to impact suspended plates with a projectile accelerated to prescribed velocities, then, taking ultrasound images of the resulting delamination. The nonlinear, multiple length-scale numerical simulations couple local crack propagation implemented through cohesive zone modeling to global stress-displacement finite element analysis. The assessment of damage uncertainty is performed in three steps by, first, considering the test data only; then, considering the simulation data only; finally, performing an assessment of total uncertainty where test and simulation data sets are combined. This study leads to practical recommendations for reducing the uncertainty and improving the prediction accuracy of the damage modeling and finite element simulation.
\end{abstract}

\footnotetext{
${ }_{1}^{1}$ Postdoctoral Research Associate, ESA-Division (ESA-WR), illucero@lanl.gov, Phone: 505-665-6236.

${ }^{2}$ Technical Staff Member, X-Division (X-1), hemez@lanl.gov, Phone: 505-667-4631.

${ }^{3}$ Faculty Affiliate, University of New Mexico, tross@lanl.gov, Phone: 505-667-6172.

${ }^{4}$ Graduate Research Assistant, ESA-Division (ESA-WR), kkline@lanl.gov, Phone: 505-663-5227.

${ }^{5}$ Technical Staff Member, ESA-Division (ESA-WR), hundhausen@lanl.gov, Phone: 505-665-1148.

${ }^{6}$ Technical Staff Member, ESA-Division (ESA-WR), tippetts@lanl.gov, E-mail: 505-667-9009.
} 
This page is left blank intentionally. 


\section{Executive Summary}

New theories have been developed to quantify uncertainty that generalize the conventional paradigm of probability theory in the context of sparse data sets, conflicting information, ambiguity, and severe epistemic uncertainty. The conventional (frequentist) interpretation of probability concerns variability, that is, information that is random in nature, and presumes that probability is an inherent physical property of an event much like mass is inherent to an object. Because the relative frequency view on probability is the limiting outcome of an experiment or observation, repeatable experiments or observations of a phenomenon must be obtained. On the contrary, the less traditional subjective probability, e.g., a Bayesian approach uses probability as degrees of belief. In the Bayesian approach, probabilities are always subjective and conditional on prior assumptions and experience. In cases where the data sets or observations are sparse, conflicting, or ambiguous, strong assumptions must be formulated to make probability theory operational. Another option is to exploit other methods of the Generalized Information Theory (GIT) that offer the advantage of relying on weaker assumptions regarding the inherent information. The five GIT methods examined in this report are probability, fuzzy sets, possibility, probability boxes, and Dempster-Shafer.

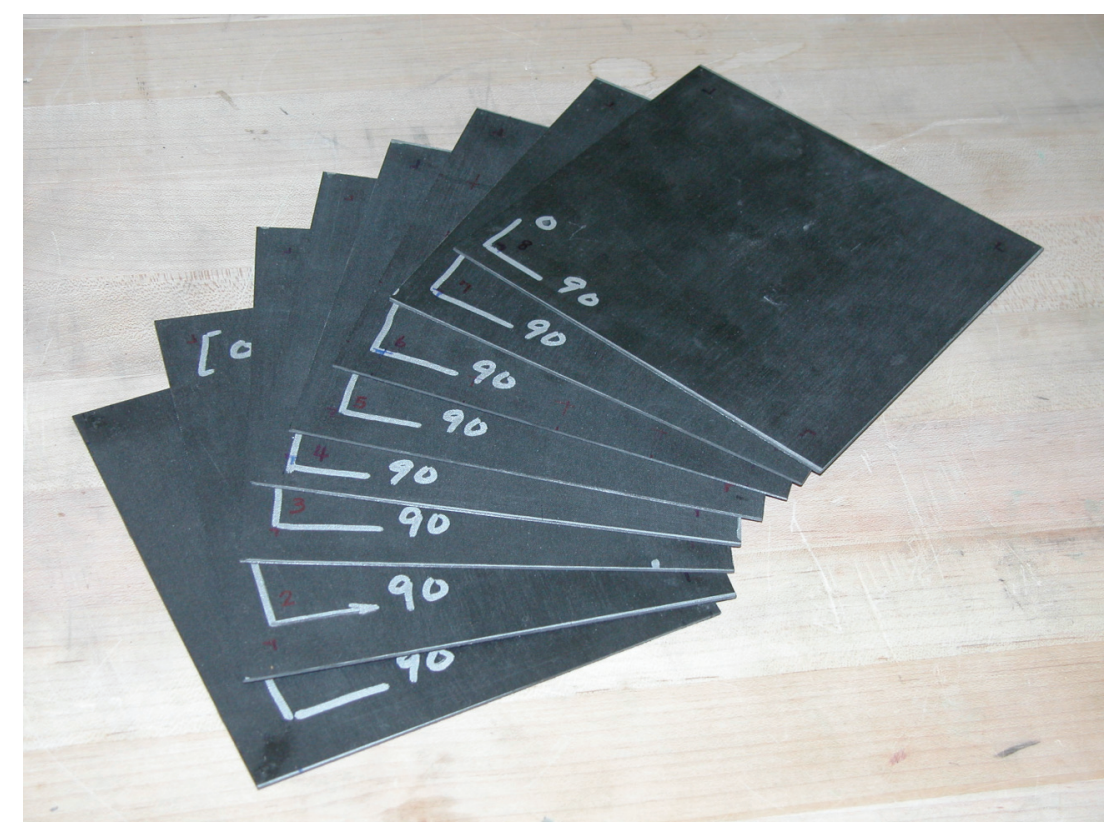

Figure 0-1. Multilayered carbon-epoxy composite plates. 
The purpose of the study is not to compare directly the different methods of uncertainty quantification but to show that they can be deployed on a practical application and to compare the assumptions upon which these theories are based. The application of interest is the prediction of delamination and fiber splitting damage induced by impacting small, multilayered carbon-epoxy composite plates with a projectile at various velocities. The composite plates have dimensions of 6-by-6 inches square, with eight plies, each 0.039 inches thick (see Figure 0-1). Each ply is comprised of a unidirectional graphite/epoxy Toray ${ }^{\mathrm{TM}}$ fiber material, with a symmetric lay-up of $+45,+90,-45,-45,+90,+45$, and 0 degrees relative to the top ply. Orthotropic material properties and their variability are obtained through dedicated coupon testing.

Gas-gun impact testing is performed by firing a hemispherically-tipped projectile at each of six similar composite plates. Exit velocity of the projectile is empirically correlated to gas-gun pressure by firing, during a series of tests on a sacrificial plate, the projectile at various pressures and measuring the exit velocity. The sacrificial tests establish that the range of impact velocities that result in the desired spread of damage severities is between $16 \mathrm{~m} / \mathrm{s}$ and $52 \mathrm{~m} / \mathrm{s}$. The second stage of the composite plate testing is to impact the five remaining plates at velocities ranging from $20 \mathrm{~m} / \mathrm{s}$ to $36 \mathrm{~m} / \mathrm{s}$. Each plate is impacted only once at its center. Six strain gauges mounted at two locations on each plate collect time-domain strain signals during impact. Although not used in the present study, the strain signals will provide useful information for future comparison with finite element predictions and model validation. Finally, ultra-sound images of the impacted plates are obtained from which delamination areas are measured using a dedicated image analysis software.

A finite element simulation is developed to predict the severity of delamination and fiber splitting damage due to projectile impact. The commercial software HKS/Abaqus implements a LANL developed nonlinear, multiscale composite damage model. This approach couples a local mesoscopic model of inter-ply and through-ply crack propagation to the global macroscopic prediction of stress and displacement states throughout the plate. The mesh is developed with 20-node quadratic hexahedra, with ten elements in each in-plane direction and one element through the thickness of each ply. This approach is capable of handling both ply splitting, that is, fracture through the ply thickness and parallel to the fibers, and delamination, that is, the disbond (separation) between adjacent plies. The ply split and delamination fracture surfaces are modeled with special contact elements that use a Cohesive Zone Model (CZM) [1] as the 
constitutive behavior. The numerical simulation introduces four main sources of uncertainty: 1) shape of the CZM ( $\left.S_{C Z M}\right)$; 2) stiffness value of the Hertz contact model between projectile and plate $\left(\mathrm{k}_{\mathrm{NL}}\right)$; 3) maximum stress that the fiber material can withstand before fracture appears $\left(\sigma_{\text {Max }}\right)$; and 4$)$ total energy that the fiber material can store before being fully separated $\left(G_{C}\right)$. These uncertainties are propagated through the simulation by means of a parametric study, which results in ranges of predicted damage areas at each level of impact velocity.

The basic data obtained by testing or simulating the plate impact responses are ranges of observed and predicted damage areas. To the aforementioned sources of testing variability and modeling lack-of-knowledge is added the ambiguity of correlating image pixel values to damage severity levels. Extreme pixel values on grayscale images indicate either no damage or full delamination, but intermediate pixel values lead to ambiguity in damage. Full delamination areas are calculated for each test or simulation, as well as ranges (intervals) of potential damage areas that account for not knowing with absolute certainty which cut-off pixel threshold should be used. The collection of intervals constitutes the processed data from which uncertainty is quantified using the five GIT approaches.

Figure 0-2 presents a brief sample of some of the types of graphical representations of uncertainty examined in this report. Using only the simulation results of the high velocity impact, graphs are constructed as either probability (PDF) or cumulative (CDF) distributions. Figure 0-2 (a) shows how a frequency distribution compares with a subjective probability approach. For example, a frequency only approach has gaps in evidence while a PDF presumes no gaps. This highlights the fundamental difference between probability approaches; frequency uses observed evidence to infer outcomes (sometimes where no evidence exists), while the subjective approach uses observations and other information to infer a continuous distribution of outcomes. Figure 0-2 (b) shows a collection of cumulative (CDF) distributions: empirical CDF, Bayes CDF, upper probability box, and lower probability box. The p-box upper and lower CDF bounds show a range on CDF values and make no assertion about the shape of a distribution. This range of distributions is one of the fundamental arguments for the use of GIT methods. Empirical data and Bayes Updating each yield a precise distribution that lie within the bounds of the p-box. Yet, many assumptions are necessary for the system in order to use just one precise distribution; but using a range of CDFs gives a more realistic portrayal of the system uncertainty. 
(a)

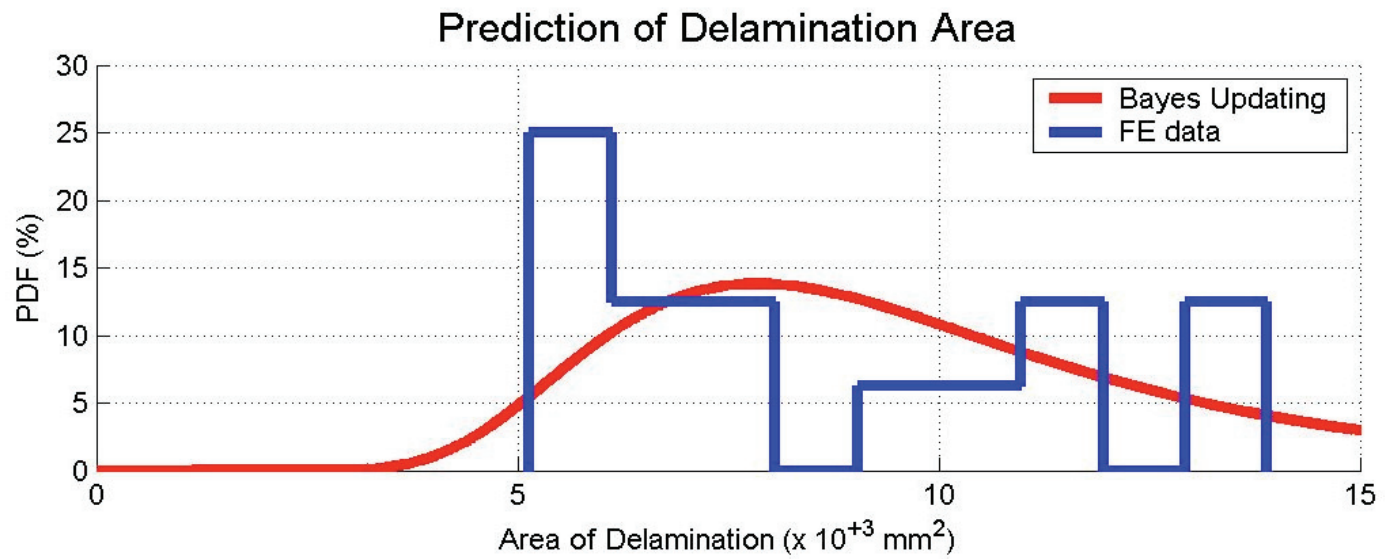

Prediction of Delamination Area

(b)

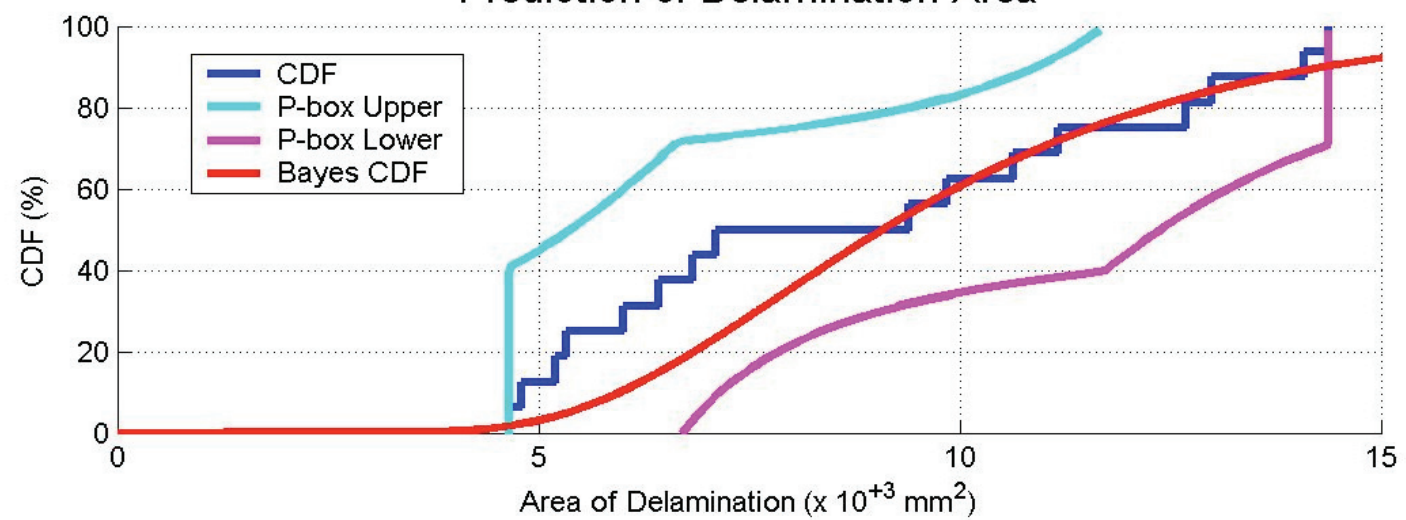

Figure 0-2. Comparison of several assessments of damage uncertainty.

The assessment of damage uncertainty is performed in three steps by, first, considering the test data only; then, considering the simulation data only; finally, performing an assessment of total uncertainty where test and simulation data sets are combined.

The main observations are the following ones. First, it is found that too few replicates of impact tests are available to enable a meaningful description of damage uncertainty using probabilistic frequencies of occurrence. This is true of physical testing where only two tests are replicated at each low and high velocity level with a single test at the medium velocity. Moreover, this sparseness of information is also true of numerical simulation where eight computer runs are performed at each of three velocity levels. Subjective probability arrives at a better quantification of damage uncertainty at the cost of formulating strong and somewhat unverified assumptions regarding the nature of underlying distribution functions. Second, the statistical analysis of variance indicates that the pair $\left(\sigma_{\mathrm{Max}} ; \mathrm{G}_{\mathrm{C}}\right)$ of fiber strength parameters is most influential in explaining how the predicted damage areas vary from one plate to another. 
Third, the fuzzy set and possibility representations of uncertainty show promise as they can be made operational in a relatively easily manner, they rely on a minimum set of assumptions, and, most importantly, they can handle various sources and types of uncertainty. Finally, imprecise probability in p-boxes and Dempster-Shafer structures offers a representation capable of encompassing the different types of uncertainty, namely, variability, ambiguity, and conflict. The advantage of using GIT methods is in providing a practical assessment capability without having to formulate somewhat subjective assumptions about the nature of uncertainty. The disadvantage is that indecision may result from such representation when the upper and lower bounds are not informative.

The overall conclusion is that representations of uncertainty provided by GIT methods such as possibility and imprecise probability should be considered to complement the tools at our disposal, tools currently restricted to frequentist and Bayesian probability representations. The GIT methods should be considered to support future programmatic applications in reliability and QMU-based (Quantification of Margin and Uncertainty) certification. Software for p-box construction could also augment current capabilities of SwRI/NESSUS and the Model Validation Toolbox for uncertainty quantification that are both used within LANL. 
This page is left blank intentionally. 


\section{Table of Contents}

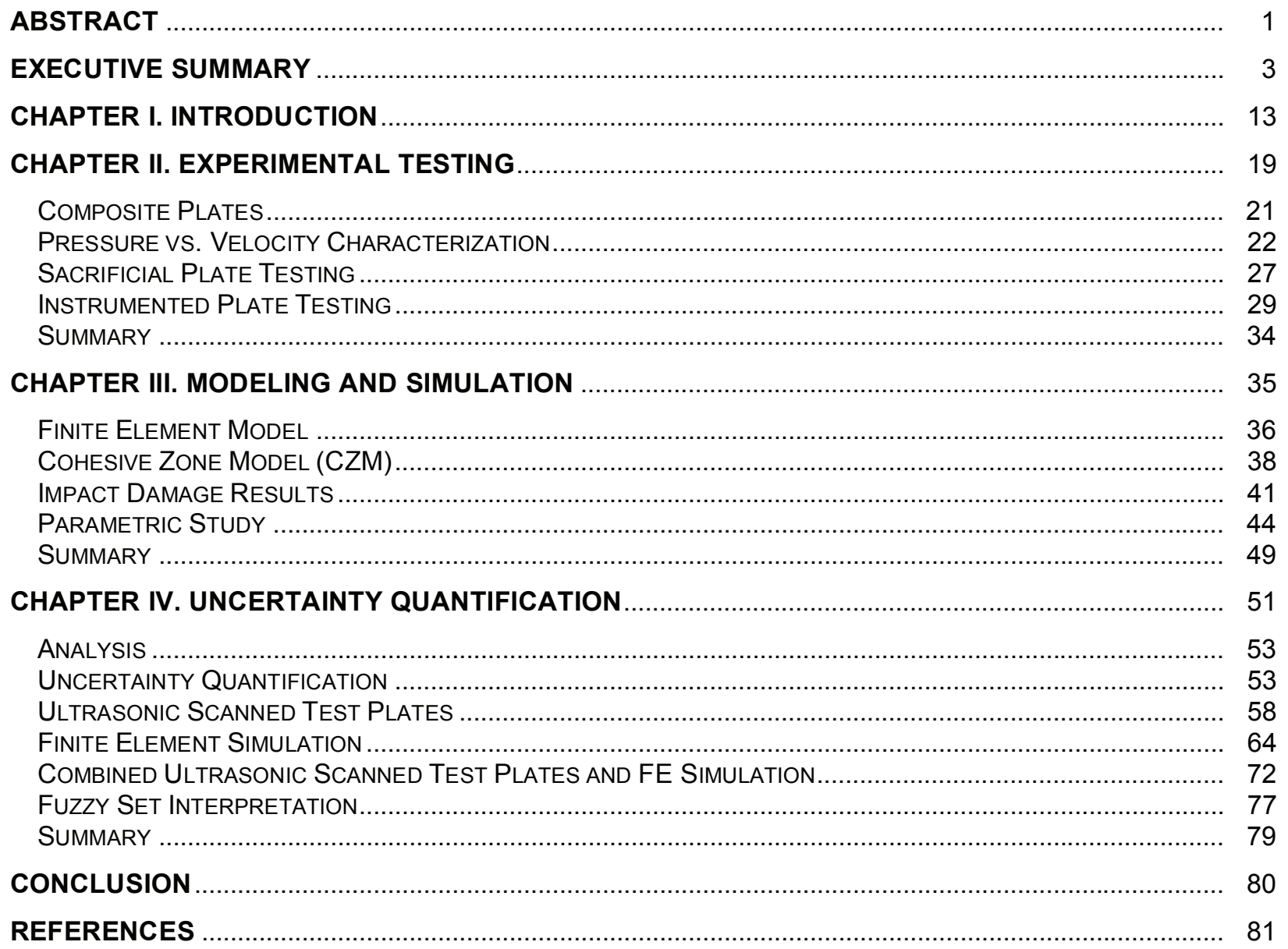




\section{Table of Figures}

Figure 0-1. Multilayered carbon-epoxy composite plates. ............................................................ 3

Figure 0-2. Comparison of several assessments of damage uncertainty..................................... 6

Figure I-1. Breakdown of features of structural damage prognosis and their

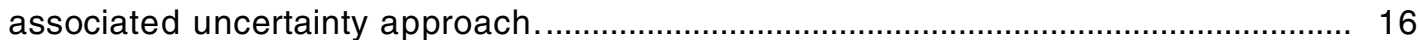

Figure II-1. Composite plate instrumented with strain gauges.................................................... 20

Figure II-2. Laminated composite plates manufactured at UCSD. ............................................. 21

Figure II-3. Ti-6Al projectile. ................................................................................................. 22

Figure II-4. Pressure vs. velocity curve for calibration tests..................................................... 23

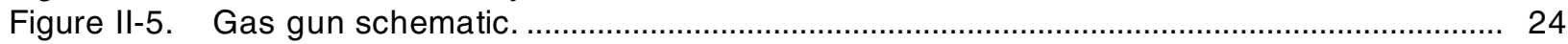

Figure II-6. Location of the projectile impact and strain gauges. ............................................. 27

Figure II-7. Instrumentation for modal testing of laminated composite plates. .............................. 29

Figure II-8. Strain gauge location and orientation................................................................ 30

Figure II-9. Plate and projectile before impact (left) and immediately following impact (right)........ 32

Figure II-10. Plot of strain vs. time for Test One, Gauge 2, 0 degrees.......................................... 32

Figure III-1. The projectile is meshed with quadratic tetrahedral elements, and the laminate plate is meshed with quadratic hexahedra elements. ............................ 37

Figure III-2. (a) Close-up of the laminate plies showing the CZM elements.

(b) Schematic of CZM elements and their location relative to the plies....................... 38

Figure III-3. Quadratic damage CZM in mode I fracture. ............................................................ 40

Figure III-4. (a) Simulation result of impact producing large bending displacements

(contours are Mises stress). (b) Snapshot from a composite plate impact

experiment showing large bending deformation.

Figure III-5. The ply split on the surface opposite impact is captured by the fracture model.

The contours are of Mises stress.

Figure III-6. A delamination forms between the last two plies opposite the impact location, oriented parallel to the ply split. The contours are of $\lambda$, the damage parameter........ 43

Figure III-7. Ultrasound C-scan shows delaminations caused by impact. ....................................... 43

Figure III-8. (a) FEM model results for parametric study run \#1. (b) FEM model results for parametric study run \#3 (delamination area shown in gray).

Figure III-9. (a) Ultrasonic scan of damaged area for velocity $=20.402 \mathrm{~m} / \mathrm{s}$.

(b) Ultrasonic scan for velocity $=32.357 \mathrm{~m} / \mathrm{s}$

47

Figure III-10. Schematic of the redesigned FEM composite plate model.

Figure III-11. Results of the redesigned FEM composite plate model results (delamination area in gray).

Figure IV-1. Possibility distribution concepts: (a) consonant interval set, (b) core interval.............. 56

Figure IV-2. Dempster-Shafer structure consisting of two focal elements. ................................... 57

Figure IV-3. Complete data set for experimental results as a frequency distribution...................... 60

Figure IV-4. Low velocity: (a) frequency dist., (b) posterior PDF from Bayesian Updating............... 61

Figure IV-5. High velocity: (a) frequency dist., (b) posterior PDF from Bayesian Updating. .............. 61

Figure IV-6. Low velocity possibility distributions: (a) from point data, (b) from interval data. ........... 62

Figure IV-7. High velocity possibility distributions: (a) from point data, (b) from interval data. ......... 62

Figure IV-8. Low velocity: (a) P-box, (b) Dempster-Shafer......................................................... 63

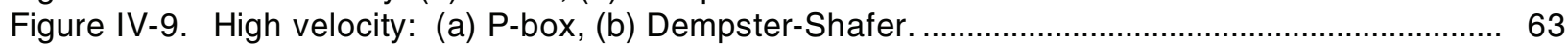

Figure IV-10. Variation of interval bounds for increasing pixel tolerance........................................6 66

Figure IV-11. Complete data set for simulation results as a frequency distribution.......................... 68

Figure IV-12. (a) Low velocity data set, (b) Bayesian Updating pdf. ............................................. 69

Figure IV-13. (a) High velocity data set, (b) Bayesian Updating pdf............................................. 69

Figure IV-14. Low velocity possibility distributions: (a) from point data, (b) from interval data. .......... 70

Figure IV-15. High velocity possibility distributions: (a) from point data, (b) from interval data. ......... 70

Figure IV-16. Low velocity (a) P-Box, (b) Dempster-Shafer structure ............................................. 71 
Figure IV-17. High velocity (a) P-Box, (b) Dempster-Shafer structure. 71

Figure IV-18. Combined plot of high impact FE predictions with corresponding test results............. 73

Figure IV-19. (a) Low velocity data set, (b) Bayesian Updating pdf. ............................................... 74

Figure IV-20. (a) High velocity data set, (b) Bayesian Updating pdf.............................................. 74

Figure IV-21. Low velocity possibility distributions: (a) from point data, (b) from interval data. .......... 75

Figure IV-22. High velocity possibility distributions: (a) from point data, (b) from interval data. ......... 75

Figure IV-23. Low velocity (a) P-Box, (b) Dempster-Shafer structure .............................................. 76

Figure IV-24. High velocity (a) P-Box, (b) Dempster-Shafer structure ........................................... 76

Figure IV-25. Fuzzy sets on the severity of delamination extent. ................................................ 78

Figure IV-26. Fuzzy sets describing delamination in terms of pixel values. ....................................... 79 


\section{List of Tables}

Table II-A. Material properties obtained from coupon testing. .................................................. 21

Table II-B. Pressure vs. velocity for the calibration tests. ........................................................ 23

Table II-C. Impact energies with various combinations of projectiles and impact velocities. ......... 26

Table II-D. Sacrificial plate test summary .............................................................................. 28

Table II-E. Instrumented plate test summary........................................................................... 31

Table II-F. Future test matrix based on 15 physical experiments.............................................. 33

Table II-G. Statistics of identified modal frequencies obtained with eight composite plates. ......... 34

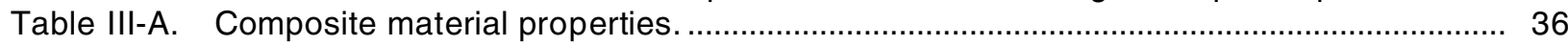

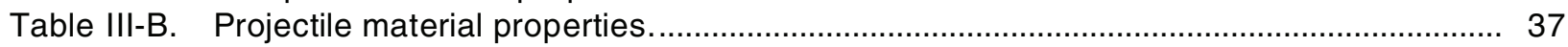

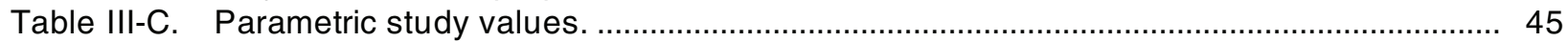

Table IV-A. Sources of uncertainty categorized into four types............................................... 52

Table IV-B. Delamination areas and associated number of pixels. ............................................ 58

Table IV-C. Delamination areas and associated number of boxes on graph paper..................... 58

Table IV-D. Delamination area intervals for each test................................................................. 59

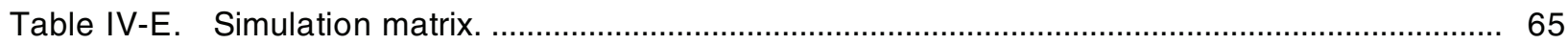

Table IV-F. Delamination areas for low projectile impact velocities and for Tolerance $=[0,40] . \ldots 67$

Table IV-G. Delamination areas for high projectile impact velocities and for Tolerance $=[0,40] \ldots 67$ 


\section{Chapter I. Introduction}

The objective of this study is to advance the capability for predicting and assessing composite laminate damage by implementing methods contained within a generalized information theory (GIT). GIT is a class of methods which extends the concept of probability theory. However, GIT relaxes some axioms of probability theory to account for sparse data, to deal with conflicting data, and to analyze system uncertainties of various types. The following GIT theories are used, probability, possibility, fuzzy sets, p-boxes, and Dempster-Shafer (as Imprecise Probability). Each of these theories has its own specific purpose in quantifying uncertainty that will be demonstrated in this report. The uncertainty that will be quantified is the uncertainty in composite laminate damage due to projectile impact.

Determining the extent of composite laminate damage in this study entails using sparse data and accommodating various types of uncertainty. This data comes from image analysis of ultrasonic scans of damaged 6 inches by 6 inches test 8-ply composite laminate plates. Additional data comes from models that predict damage using the general purpose finite element (FE) software HKS/Abaqus ${ }^{T M}$. These data values represent delamination or the separation of adjacent plies and will be used as point values and interval values in the various GIT methods. This study also has a significant impact on structural damage prognosis.

Structural damage prognosis is a complex field of study that incorporates physics based models to simulate the initiation and evolution of damage in advanced materials. This research on composite laminate plates will contribute significantly to the improvement of physics based modeling. But in order to make this improvement, an analysis of recent theories to quantify uncertainty is critical. To get a better understanding of physics based models requires a class of theories that addresses uncertainty from a wider perspective than traditional probability theory.

Probability theory is as much a science as it is a philosophy. In its simplest terms, probability is simply evidence supporting a proposition. This evidence is quantified as a measure called a probability measure. A probability measure is a function that maps outcomes to values on the unit interval. This vague notion of probability as evidence begins the controversy of what constitutes evidence and is central to the evolution of various probability approaches (e.g. frequentist, Bayesian and others). 
The concept of probability is evolving from its original intent as a measure for games of chance. The classical view of probability theory concerns information that is random and presumes that probabilities exist inherently in nature. In other words, the number of favorable outcomes divided by the total number of possible outcomes yields the probability. However, if we remove ourselves from this absolute notion we find the Relative Frequency view. The Relative Frequency view is more closely associated with the idea, "law of large numbers." In this view, probability is the limiting outcome of an experiment and is not an independent existence; it's the ratio of favorable outcomes to unfavorable. This is fine when we have repeatable experiments or phenomena. When this is not the case, we must remove ourselves further to the realm of the Propensity view. This is one of the largest conceptual leaps in probability theory and was due to Karl Popper around 1959. This view concerns unrepeatable phenomena where probability is more a disposition or tendency of nature. Causal models of the physical system are used to estimate knowledge where randomness is not an objectively measurable phenomenon but a "knowledge" phenomenon. Here we see the first attempts of incorporating the uncertainty associated with the knowledge about the physical system. As such, probabilities are really a measure of "lack of knowledge" about the affecting conditions. Yet, Popper's point of view still contends that probability is objective. Around the same time (1954), Leonard Savage axiomatized the "Ramsey-de Finetti" view of probabilities to be subjective, representing personal belief. This view ties together choice and uncertainty. The Ramsey-de Finetti view states that personal beliefs can be inferred from observed bets. In other words, subjective probabilities are beliefs based on personal actions. But there exists another perspective on subjective probability. The "intuitionist" view of probability maintains that probabilities are subjective beliefs, however probabilities are prior to experience and contrast the "Ramsey-de Finetti" view. This approach was developed by B.O. Koopman (1940) and Irving J. Good (1962) and most closely resembles the GIT methods that are researched today. In this perspective, subjective probability assignments aren't always revealed through choice; but if they are then the probabilities are interval rather than single values and are partially ordered.

Another advance to subjective probability uses the Bayesian procedure for updating prior probabilities, namely Bayes Theorem. The Bayesian approach is the one most used in the scientific method. Yet the scientific method can benefit greatly from more recent theories of uncertainty quantification. 
The standard axioms for probability theory are the following:

$$
\begin{array}{ll}
\text { I. } & P(A) \geq 0 . \\
\text { II. } & P(X)=1 . \\
\text { III. } & \text { For any sequence of disjoint sets } A_{i} \in X: P\left(\cup A_{i}\right)=\Sigma P\left(A_{i}\right) \text { (additivity) } \\
\text { IV. If } P(B)>0 \text {, then } P(A \mid B)=P(A \& B) / P(B)
\end{array}
$$

$P()$ is the probability measure. A and $B$ are events or sets in the universe of discourse (domain), X.

One of the most controversial assertions in the Bayesian framework is that one's prior degree of belief doesn't matter. The Bayesian assertion is that what matters is how the degree of belief is modified. Belief is the Posterior Probability after being updated with evidence of a hypothesis about its prior probability.

$$
P(B \mid A)=\frac{P(B) * P(A \mid B)}{P(A)}
$$

However, we know that the beginning degree of belief matters a lot especially in situations with sparse data or when the uncertainty comes in various forms.

Figure I-1 identifies a few types of uncertainties that contribute to structural damage prognosis. Structural Damage Prognosis is comprised of three components: coupon testing, physics based modeling, and large-scale simulation. A successful structural damage prognosis paradigm cannot be achieved without all three aspects. And to each aspect there exists an appropriate theory to address the uncertainty. For example, the relative frequency approach to probability can appropriately accommodate coupon testing; the material properties that are used in a particular design are based on an aggregation of many property tests. These tests can be considered to be repeatable and under good experimental control. As such, a frequency approach to their probability assessments might be sufficient. This means that a sufficient amount of data can be accumulated to support the claim that a limiting outcome (probability) is achievable. A physics based model relies on the coupon testing for input parameter values. However, information for physics based modeling grows in complexity, for example the load impact or distribution. The distribution of load in a structure can only be assessed vaguely. Some limiting conditions are presumed, but not all conditions are known beforehand. This means that the repeatability of experiments is very unlikely or the data retrieved from such experiments is very small. So the tendency of probabilities can only be estimated. In other 
words, the reliance on models to make inferences based on the causal relations is used to develop the probabilities with little experimental verification. As such, a propensity or subjective approach is necessary because it relies on a more loose interpretation of the assignment of probabilities. Moreover, to perform experimental tests also requires much effort and under less controllable conditions. Therefore, a propensity or subjective approach can handle this component appropriately. Finally, we come to the most nebulous form of information for structural health or damage prognosis, large-scale simulation. This form of information is unique to each structure. Because of this uniqueness, no repeatable experiments or data are available; this form of information is rare at best but most often is not available. In the event that two structures add to the same data base, they are still unique because they were designed and built under different (but very similar) conditions. This means that a reliance on some partial logical inference makes the most sense. Causal models are required to estimate feature response and a structure's state of health. The recent theories of imprecise probabilities address this case specifically in accumulating evidence. Clearly, as the repeatability of data is decreased there is more reliance on model simulation. To deal with each type of information the same way is not the best paradigm; a non-additive approach is needed that makes no inference on the space of possibilities but uses only the evidence at hand as a one to many mapping to make predictions in the simulations.

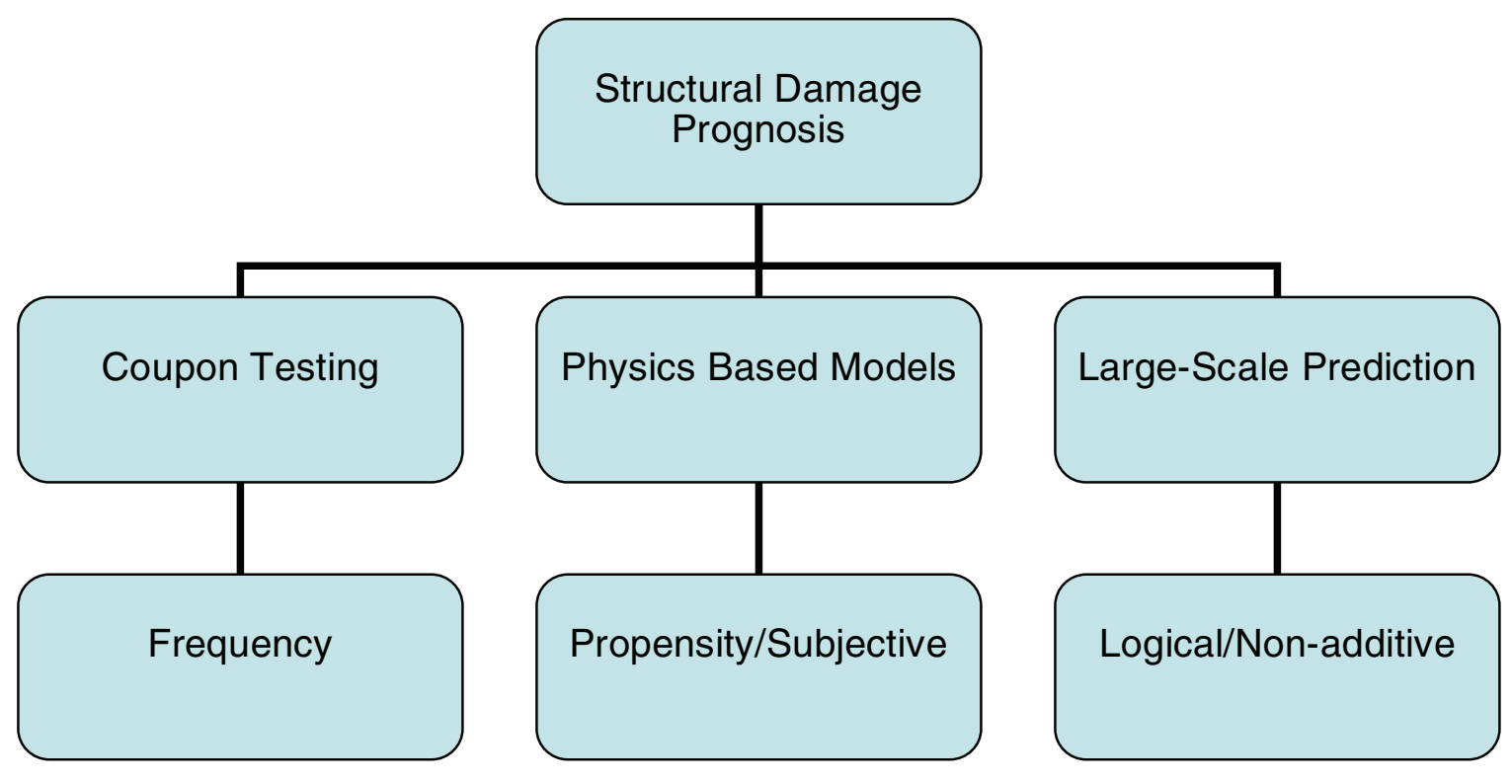

Figure I-1. Breakdown of features of structural damage prognosis and their associated uncertainty approach. 
Most of the time, especially in engineering systems, there is insufficient information to identify a unique probability distribution. In these cases, techniques of Generalized Information Theory can be applied in order to represent better the available system knowledge. The phrase Generalized Information Theory does not imply just one theory; it defines a class of methods that avoid the limitations of traditional probability theory. We identify three categories of uncertainty sources and four categories of uncertainty types. The sources are related to the individual components of this study and include image analysis, experimental and simulation. Specifically associated with image analysis is the uncertainty due to the pixel value and its threshold for delamination, the ultrasonic scan and the noise the scan generates. Moreover, the uncertainties associated with the experiments are the set-up, plate specimens, and projectile velocity. Finally, the FE simulations come with model uncertainties concerning the boundary conditions, the CZM interaction, and the contact stiffness between the projectile and the plate. Additionally, the FE simulation has uncertainty associated with a calculated damage value. The damage severity is calculated on the unit interval and, like the pixel values, the threshold between full delamination and no delamination is unclear, or fuzzy. Associated with each of these uncertainty sources is the type of uncertainty.

A particular source of uncertainty may be due to ambiguity, randomness, nonspecificity, or interpretation. For example, the meaning of partial damage in the FE simulation is ambiguous. Moreover, since the test projectile has many unseen nonlinear effects imparting forces on it before it hits the plate, there are nonspecificity and random uncertainties associated with its velocity. Finally, the ultrasonic scans are ambiguous and have uncertainty associated with calculating the damage area. We need a tool that improves upon traditional probability theory to quantify disparate uncertainty. 
This page is left blank intentionally. 


\section{Chapter II. Experimental Testing}

The purpose of these impact tests of small composite plates is to provide data for the Verification and Validation ( $\mathrm{V} \& \mathrm{~V}$ ) of the composite model implemented in the general purpose finite element software HKS/Abaqus ${ }^{\mathrm{TM}}$ in support of the Damage Prognosis project. The assessment of validation requires the comparison between predictions of numerical simulations and physical measurements of the area of delamination and fiber splitting that results from impacting the composite plates with a projectile. The primary response feature is an indicator of the area of delamination obtained through post-shot ultrasonic inspection. Strain measurements will also be collected during the impact tests to provide other comparison metrics if necessary. To validate the composite model, it is necessary to perform impact tests at several levels of impact velocity. The impacts must also be replicated to estimate the testing and environmental variability. This experimental data set will also support the quantification of uncertainty using the General Information Theory (GIT), in support of the ASC Engineering V\&V Program.

Gas-gun impact testing was performed by firing a hemispherically-tipped Ti-6Al projectile at each of six composite plates. The composite plates, which had nominal dimensions of 6-inches by 6 -inches by 0.039 -inches $\left(1-\mathrm{mm}\right.$ ) thick (Figure II-1), were made from eight layers of Toray ${ }^{\mathrm{TM}}$ fiber laid in $[0,45,90,-45]$ degree orientations, symmetric about the mid-plane of the plate.

Initially, a correlation between the gas-gun pressure and the exit velocity of the Ti-6Al projectile needed to be established. This correlation was derived empirically by firing the projectile at various pressures and measuring the exit velocity. In all, 11 calibration shots were fired and the relationship between pressure and velocity was established over a range of projectile velocities between approximately 16 and $52 \mathrm{~m} / \mathrm{s}$. It was believed that this range of velocities would encompass those velocities that would be used in the impact testing.

The impact testing was divided into two stages. In the first stage, a sacrificial plate was impacted five times at projectile velocities ranging from approximately 21 to $36 \mathrm{~m} / \mathrm{s}$. The purpose of the sacrificial plate testing was to determine an appropriate range of velocities that would introduce damage at three desired levels as observed without the use of ultrasonic scanners or other nondestructive evaluation (NDE) techniques. Specifically, these damage levels were categorized as follows: "Level 1" damage, meaning damage that was very difficult to see; "Level 2" damage that was difficult, but possible, to see; and "Level 3" damage that was readily identifiable. It was found that Level 1 damage could be introduced with projectile 
velocities of $20-22 \mathrm{~m} / \mathrm{s}$, Level 2 damage could be achieved with $28-32 \mathrm{~m} / \mathrm{s}$ impact velocities, and Level 3 damage could be introduced by impacting at $36-38 \mathrm{~m} / \mathrm{s}$. Each impact was performed at a different location on the sacrificial plate so as to inhibit damage zones from adjacent impacts from converging.

The second stage of the composite plate impact testing was the testing of plates that had been instrumented with strain gauges (Figure II-1). These strain gauges were used to collect strain data in three directions (0-, 45-, and 90-degrees) at two locations on each plate. These data were collected for comparison with the simulation results from a finite element model of the projectile/plate impact event. In all, five impacts were performed using the aforementioned Ti-6Al projectile at velocities ranging from approximately 20 to $36 \mathrm{~m} / \mathrm{s}$. Each plate was impacted only once, and the impact was performed at the center of the plate.

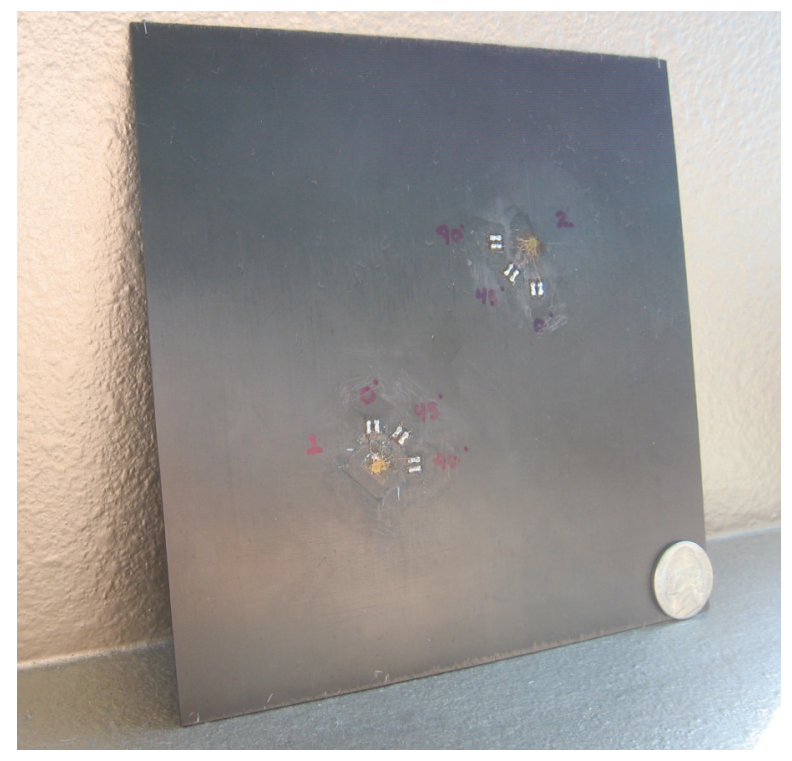

Figure II-1. Composite plate instrumented with strain gauges.

The data collected from the instrumented plates showed that the strain gauges were destroyed shortly after impact. This outcome was expected, and the data collected in the brief amount of time between impact and loss of signal was generally high quality. There were several instances where it was apparent that the jumper wires, which connect the strain gauge wires to the data acquisition system wires, had instantaneous periods where they were shorting together. This shorting of the jumper wires, which showed up in the data as sharp spikes, was caused by the severe shock waves that were propagating through the plate during impact; however, given the exceedingly short amount of time that the jumper wires were in contact, these spikes in the data could be assuaged by post-test signal processing. 


\section{Composite Plates}

The plates were manufactured at the University of California at San Diego (UCSD) and are shown in Figure II-2. The plates were 152.4 mm (6.0 inch) square, $1.0 \mathrm{~mm}$ (0.04 inch) thick, and made of eight orthotropic carbon fiber plies; each ply was $0.127 \mathrm{~mm}$ (0.005 inch) thick. The ply orientation angles were $[0 ; 45 ; 90 ;-45 ;-45 ; 90 ; 45 ; 0]$ degrees. The material properties are defined in Table II-A.

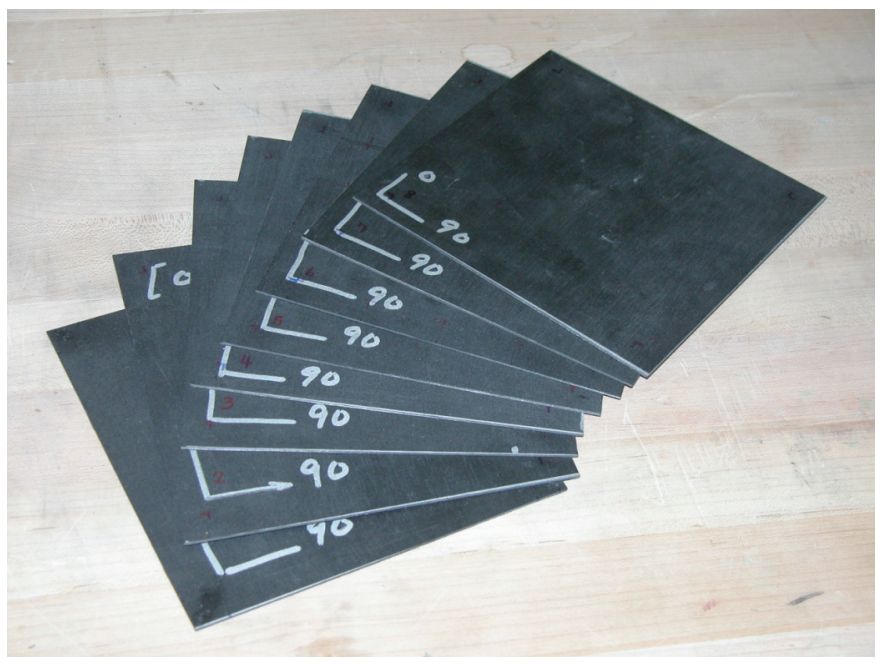

Figure II-2. Laminated composite plates manufactured at UCSD.

Table II-A. Material properties obtained from coupon testing.

\begin{tabular}{|c|c|c|}
\hline Symbol & Mean ( ) & Standard Deviation $(\sigma)$ \\
\hline$E_{11}$ & $132.4 \times 10^{+9} \mathrm{~N} / \mathrm{m}^{2}$ & $3 \%$ of mean \\
\hline$E_{22}=E_{33}$ & $9.1 \times 10^{+9} \mathrm{~N} / \mathrm{m}^{2}$ & $2 \%$ of mean \\
\hline $\mathrm{G}_{12}=\mathrm{G}_{13}$ & $4.5 \times 10^{+9} \mathrm{~N} / \mathrm{m}^{2}$ & $3.6 \%$ of mean \\
\hline $\mathrm{G}_{23}$ & $3.0 \times 10^{+9} \mathrm{~N} / \mathrm{m}^{2}$ & Unknown \\
\hline$v_{12}=v_{13}$ & 0.30 & Unknown \\
\hline$v_{23}$ & 0.40 & Unknown \\
\hline$\rho$ & $1,522.0 \mathrm{~kg} / \mathrm{m}^{3}$ & $2.5 \%$ of mean \\
\hline
\end{tabular}




\section{Pressure vs. Velocity Characterization}

Characterization of the pressure vs. velocity (P-V) curves was performed with a hemispherically-tipped Ti-6Al projectile that had a mass of 25.458 grams. The nominal dimensions of the projectile are shown in Figure II-3.

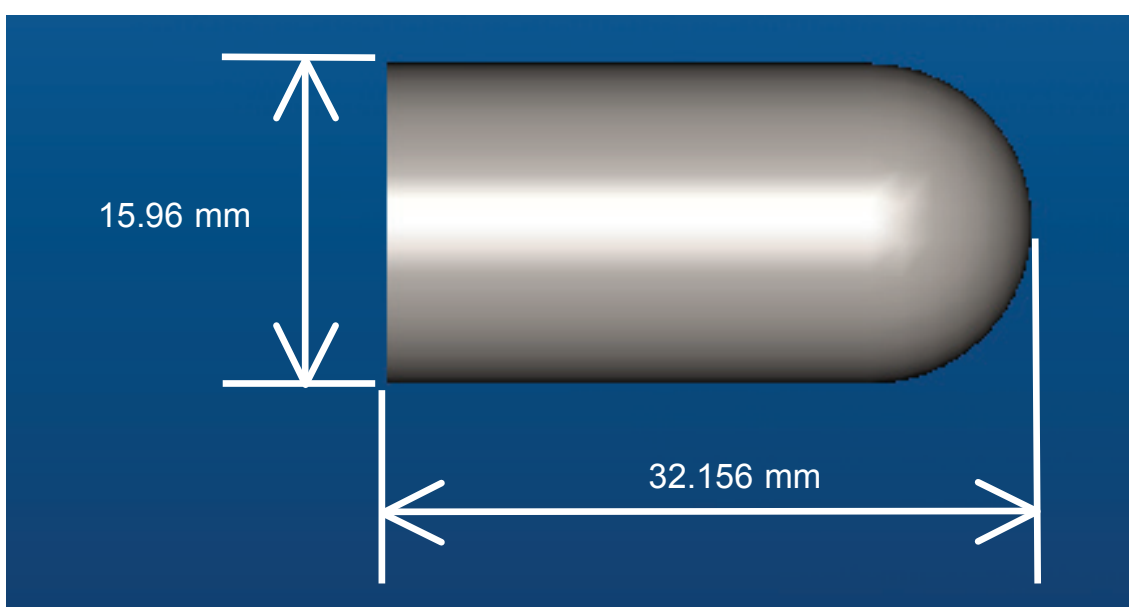

Figure II-3. Ti-6AI projectile.

A total of 11 calibration tests were performed by firing the projectile out of a gas gun at pressures varying from $4.02 \mathrm{psi}$ to $24.98 \mathrm{psi}$. It was believed that 11 tests would provide enough data points to produce a reasonable trend of pressure vs. velocity over the range of the pressures anticipated to be used during the sacrificial plate testing. The velocity of the projectile was measured with a laser timing gate. A Tektronix TDS460A digital oscilloscope was used to measure the time between the interruption of the first laser beam and the interruption of the second laser beam. By dividing the distance between the two beams by the amount of time required to break each beam, an estimate of the projectile velocity (denoted "2-Gate Velocity") could be made. Table II-B details the pressures and corresponding velocities for the 11 calibration tests. 
Table II-B. Pressure vs. velocity for the calibration tests.

\begin{tabular}{|c|c|c|c|c|c|c|c|}
\hline Shot & $\begin{array}{c}\text { Projectile } \\
\text { Mass (gm) }\end{array}$ & $\begin{array}{c}\text { Projectile } \\
\text { Length } \mathbf{( m m} \text { ) }\end{array}$ & $\begin{array}{c}\text { Press. } \\
\mathbf{( p s i )}\end{array}$ & $\begin{array}{c}\text { 2-Gate } \mathbf{t} \\
\mathbf{( m s )}\end{array}$ & $\begin{array}{c}\text { 2-Gate } \\
\text { Velocity } \mathbf{( m / s})\end{array}$ & $\begin{array}{c}\text { 1-Gate } \\
\mathbf{t}(\mathbf{m s})\end{array}$ & $\begin{array}{c}\text { 1-Gate Velocity } \\
(\mathbf{m} / \mathbf{s})\end{array}$ \\
\hline 1 & 26.458 & 32.1564 & 10.08 & 3.26 & 31.166 & 1.02 & 31.526 \\
\hline 2 & 26.458 & 32.1564 & 7.02 & 4.06 & 25.025 & 1.30 & 24.736 \\
\hline 3 & 26.458 & 32.1564 & 4.02 & 6.02 & 16.877 & 1.88 & 17.104 \\
\hline 4 & 26.458 & 32.1564 & 5.50 & 4.74 & 21.435 & 1.48 & 21.727 \\
\hline 5 & 26.458 & 32.1564 & 5.05 & 4.96 & 20.484 & 1.52 & 21.156 \\
\hline 6 & 26.458 & 32.1564 & 5.51 & 4.76 & 21.345 & 1.48 & 21.727 \\
\hline 7 & 26.458 & 32.1564 & 6.02 & 4.34 & 23.410 & 1.36 & 23.644 \\
\hline 8 & 26.458 & 32.1564 & 8.49 & 3.61 & 28.144 & 1.11 & 28.970 \\
\hline 9 & 26.458 & 32.1564 & 15.02 & 2.60 & 39.077 & 0.80 & 40.196 \\
\hline 10 & 26.458 & 32.1564 & 20.01 & 2.16 & 47.037 & 0.67 & 47.995 \\
\hline 11 & 26.458 & 32.1564 & 24.98 & 1.93 & 52.642 & 0.60 & 53.594 \\
\hline
\end{tabular}

A second measurement of the velocity was calculated by measuring the amount of time required for one laser beam to be broken and subsequently reestablished; this velocity measurement (denoted "1-Gate Velocity") was calculated by dividing the length of the projectile by the amount of time elapsed between breaking and reestablishment of the beam. The reason for the difference between the 2-Gate Velocity and the 1-Gate Velocity was because the 1-Gate Velocity was measured using the second laser timing gate, i.e., it was closer to the end of the barrel and subsequently had more time to accelerate. It is unknown why the 1-Gate Velocity in shot two is lower than the corresponding 2-Gate Velocity. Figure II-4 shows the P-V chart for the calibration tests where a nonlinear relationship exists; this nonlinear trend matches in shape the theoretical model for exit velocity.

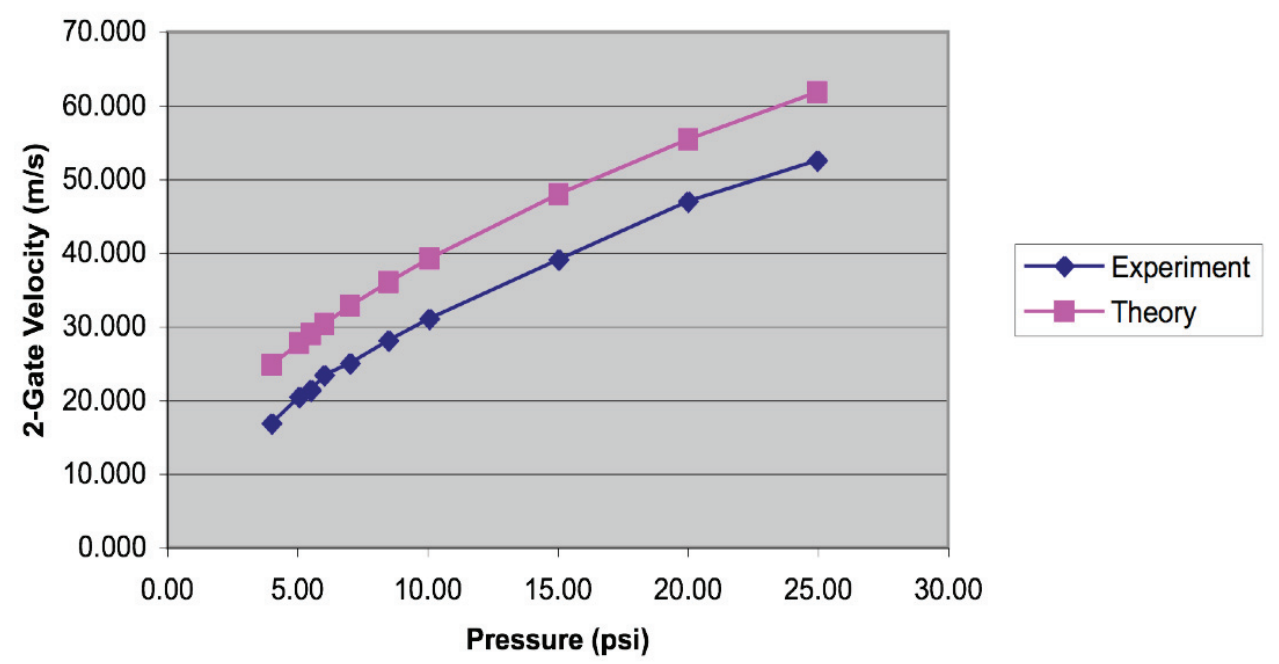

Figure II-4. Pressure vs. velocity curve for calibration tests. 
The following section is a derivation of the exit velocity for the experiments. Figure II-5 is a schematic of the gas gun set up with the corresponding barrel lengths and velocities.

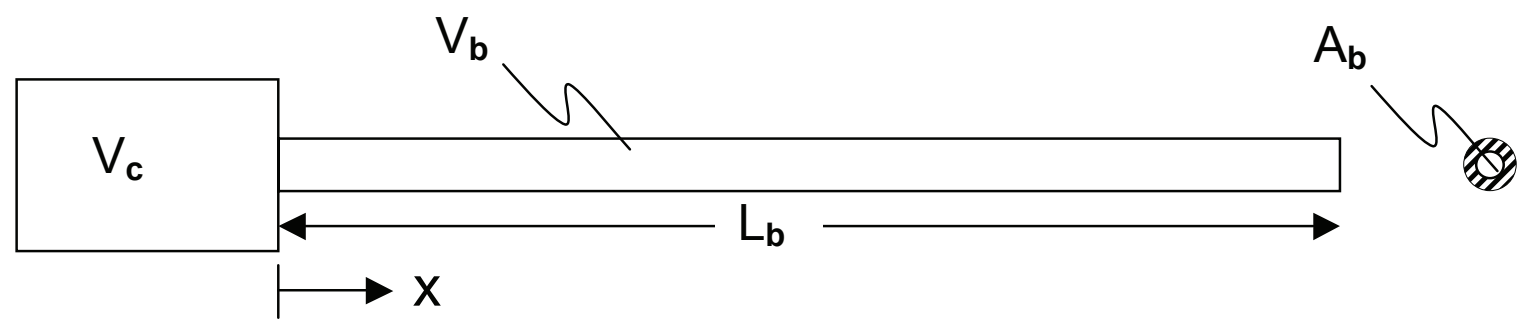

Figure II-5. Gas gun schematic.

Nomenclature:

\begin{tabular}{|l|l|}
\hline$V_{c}=$ Volume of Chamber & $P_{i}=$ Initial Pressure \\
\hline$V_{b}=$ Volume of Barrel & $P_{f}=$ Final Pressure \\
\hline$L_{b}=$ Length of Barrel & $F_{i}=$ Initial Force on Projectile \\
\hline$A_{b}=$ Area of Barrel & $F_{f}=$ Final Force on Projectile \\
\hline$m=$ Mass of Projectile & $V_{f}=$ Final (Exit) Velocity \\
\hline
\end{tabular}

By a summation of forces, we obtain the following equation.

$$
\begin{aligned}
& F_{i}=P_{i} A_{b} \\
& F_{f}=P_{i} A_{b}\left(\frac{V_{c}}{V_{c}+V_{b}}\right) \\
& F(x)=F_{i}-\frac{F_{i}-F_{f}}{L_{b}} x
\end{aligned}
$$

Next, we use Newton's $2^{\text {nd }}$ Law and apply the Chain Rule.

$$
F(x)=m \frac{d V}{d t}=m \frac{d V}{d x} \frac{d x}{d t}=m V \frac{d V}{d x} \quad * V=\text { Velocity }
$$


Finally, we integrate to obtain the final velocity, $V_{f}$.

$$
\begin{aligned}
& \int_{0}^{L_{b}} F(x) d x=m \int_{0}^{V_{f}} V d V \\
& \int_{x=0}^{x=L_{b}}\left(F_{i}-\frac{F_{i}-F_{f}}{L_{b}} x\right) d x=m \int_{V_{i}}^{V_{f}} V d V ; V_{i}=0 ; \\
& \frac{1}{2} L_{b}\left(F_{i}+F_{f}\right)=\frac{1}{2} m V_{f}^{2} \\
& V_{f}=\left[\frac{L_{b}\left(F_{i}+F_{f}\right)}{m}\right]^{\frac{1}{2}}=\left[\frac{A_{b} L_{b} P_{i}}{m}\left(\frac{V_{c}}{V_{c}+V_{b}}+1\right)\right]^{\frac{1}{2}}
\end{aligned}
$$

It is believed that the bias difference between the two P-V curves is the result of barrel/projectile friction and air resistance, both of which were, for simplicity, neglected in the theoretical derivation.

The nominal projectile was cylindrically-shaped, $25.4 \mathrm{~mm}$ (1.0 inch) in diameter, $50.8 \mathrm{~mm}$ (2.0 inches) in length with a hemispherical end on the impacting side. Options available to design the projectile and select the impact velocity levels are discussed below.

A similar impact study on small composite plates was conducted by Taylor et al. [2]. However in their work, they used nylon not aluminum projectiles at velocities ranging from $V_{1}=29.0 \mathrm{~m} / \mathrm{s}$ to $V_{1}=53.0 \mathrm{~m} / \mathrm{s}$. The nylon projectile was cylindrically-shaped with a hemispherical end on the impacting side; its length and diameter were $L=50.8 \mathrm{~mm}$ (2.0 inches) and $D=12.7 \mathrm{~mm}(1 / 2$ inch), respectively. The calculation of the projective volume is given in Equation (II-1).

$$
\text { Vol }=\underbrace{\left(L-\frac{D}{2}\right) \pi\left(\frac{D}{2}\right)^{2}}_{\text {Cylinder Volume }}+\underbrace{\frac{1}{2} \cdot \frac{3}{4} \pi\left(\frac{D}{2}\right)^{3}}_{\text {Half SphereVolume }}=\frac{\pi D^{2}}{4}\left(L-\frac{D}{6}\right)
$$

where $L$ is the length of the projectile and $D$ is the diameter. The mass and kinetic energy of the projectile are given by Equations (II-2) and (II-3), respectively:

$$
\begin{aligned}
& M=\rho V o l=\frac{\pi \rho D^{2}}{4}\left(L-\frac{D}{6}\right) \\
& E=\frac{1}{2} M V_{l}^{2}
\end{aligned}
$$


where $\rho$ denotes the material density. With dimensions $L=50.8 \mathrm{~mm}$ and $D=12.7 \mathrm{~mm}$, the volume is calculated to be $\mathrm{Vol}=6,167.0 \mathrm{~mm}^{3}$. Since mass is known to be $M=0.031 \mathrm{~kg}$, the density of nylon material is back-calculated and equal to $\rho=5,026.72 \mathrm{~kg} / \mathrm{m}^{3}$. As a result, impact energies are calculated to be $E=13.03 \mathrm{~J}(1 \mathrm{~J}=1 \mathrm{Nm})$ at the impact velocity of $V_{1}=29.0 \mathrm{~m} / \mathrm{s}$; and $\mathrm{E}=43.44 \mathrm{~J}$ at $\mathrm{V}_{\mathrm{l}}=53.0 \mathrm{~m} / \mathrm{s}$.

Taylor et al. [2] reported that the high impact velocity levels (above $50.0 \mathrm{~m} / \mathrm{s}$ ) result in significant delamination and fiber splitting damage, visible from one edge of the plate to the other. Therefore in our study it seemed undesirable to exceed the impact energy of $40 \mathrm{~J}$. Several options for designing the projectile and selecting impact velocity levels are explored in Table II-C. The main constraints for designing the projectile and selecting the velocity levels are:

1. Keep the impact energy given by Equation (II-3) less than $40 \mathrm{~J}$;

2. Keep the impact velocity as high as possible (above $20 \mathrm{~m} / \mathrm{s}$ ) to make the shots as repeatable as possible;

3. Keep the mass of the projectile below 38 grams, if possible, to make it less heavy than the composite plate, hence, avoiding double hits.

Table II-C. Impact energies with various combinations of projectiles and impact velocities.

\begin{tabular}{|c|c|c|c|}
\hline \multirow{2}{*}{ Option 1} & \multicolumn{3}{|c|}{ 2-inch Long, $1 / 2$-inch Diameter, Nylon Projectile } \\
\hline & $V_{1}=20.0 \mathrm{~m} / \mathrm{s}$ & $V_{1}=35.0 \mathrm{~m} / \mathrm{s}$ & $V_{1}=50.0 \mathrm{~m} / \mathrm{s}$ \\
\hline $\begin{array}{l}\text { Length, } L=50.8 \mathrm{~mm} \\
\text { Diameter, } D=12.7 \mathrm{~mm} \\
\text { Volume, } V o l=6,167.0 \mathrm{~mm}^{3} \\
\text { Density, } \rho=5,026.7 \mathrm{~kg} / \mathrm{m}^{3} \\
\text { Mass, } M=0.031 \mathrm{~kg}\end{array}$ & $E=6.2$ Joules & $E=19.0$ Joules & $E=38.7$ Joules \\
\hline \multirow{2}{*}{ Option 2} & \multicolumn{3}{|c|}{ 1-inch Long, $1 / 2$-inch Diameter, Steel Projectile } \\
\hline & $V_{1}=30.0 \mathrm{~m} / \mathrm{s}$ & $V_{1}=45.0 \mathrm{~m} / \mathrm{s}$ & $V_{1}=60.0 \mathrm{~m} / \mathrm{s}$ \\
\hline $\begin{array}{l}\text { Length, } L=25.4 \mathrm{~mm} \\
\text { Diameter, } D=12.7 \mathrm{~mm} \\
\text { Volume, } \mathrm{Vol}=2,949.5 \mathrm{~mm}^{3} \\
\text { Density, } \rho=7,800.0 \mathrm{~kg} / \mathrm{m}^{3} \\
\text { Mass, } M=0.023 \mathrm{~kg}\end{array}$ & $E=10.3$ Joules & $E=23.3$ Joules & $E=41.1$ Joules \\
\hline
\end{tabular}




\section{Sacrificial Plate Testing}

The purpose of the sacrificial plate testing was to determine the appropriate range of test velocities given the three desired levels of damage: "Level 1," meaning the damage was very difficult to detect with the naked eye; "Level 2," meaning the damage was possible, but difficult, to detect with the naked eye; and "Level 3," meaning delamination of the layers was readily identifiable.

The test environment was designed to isolate the response of the projectile-plate impact as much as possible while allowing for video recording. The same Ti-6Al projectile used in the P-V characterization was used on the sacrificial plate. In all, five tests were performed with projectile velocities ranging from $20.098 \mathrm{~m} / \mathrm{s}$ to $39.759 \mathrm{~m} / \mathrm{s}$. The plate was suspended inside a steel box with monofilament lines attached to small holes drilled in the upper corners of the plate (see Figure II-6). One side of the steel box was made of a 0.5 -inch polycarbonate panel-this ensured that personnel and equipment were protected from flying debris while allowing for a high-speed video camera to record the event. The camera used was the Phantom 5 , made by Vision Research. The frame rate was 22,222 frames per second with a $10 \mathrm{~s}$ exposure time at a resolution of 256 by 128 pixels. Care was taken to ensure that the same point on the plate was not impacted more than once.

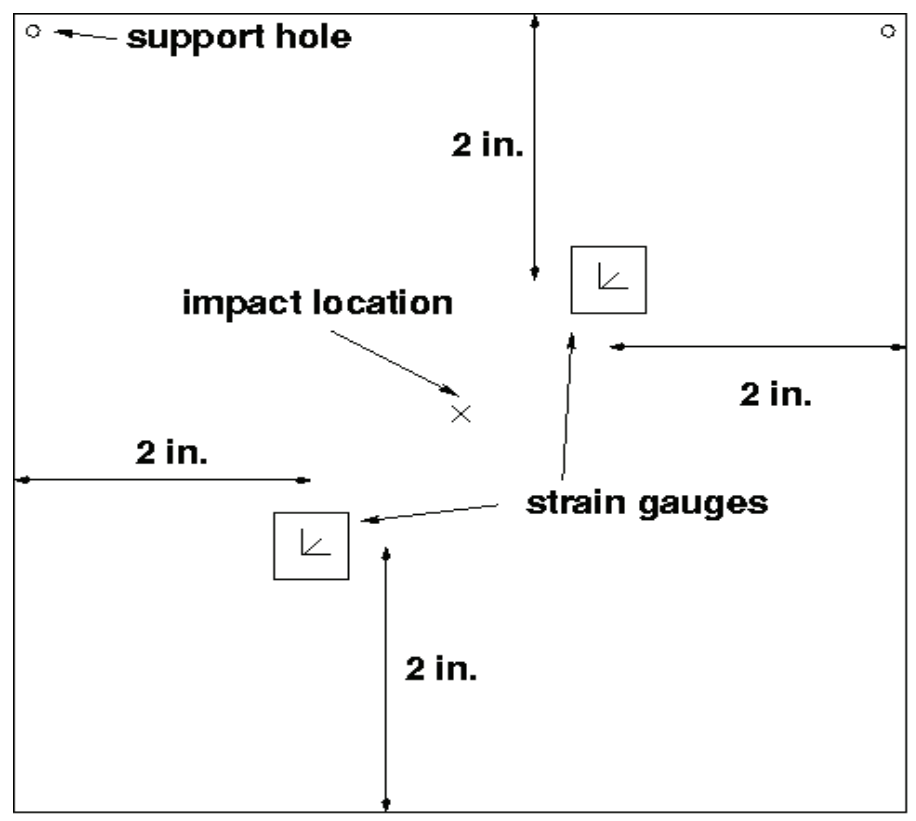

Figure II-6. Location of the projectile impact and strain gauges. 
Table II-D details the operating pressures, impact velocities and locations (as viewed from the impact side of the plate), and a brief description of the visible damage caused thereon.

Given the results of the sacrificial plate testing, it was determined that Level 1 (low-level) damage could be introduced by impacting the plate at approximately $20-22 \mathrm{~m} / \mathrm{s}$, Level 2 at 28-32 m/s, and Level 3 damage at 36-38 m/s. It is important to note that the damage levels were determined solely by a visual inspection of the plate following the impact.

Table II-D. Sacrificial plate test summary.

\begin{tabular}{|c|c|c|c|c|}
\hline $\begin{array}{c}\text { Test } \\
\text { (Filename) }\end{array}$ & $\begin{array}{l}\text { Pressure } \\
\text { (psi) }\end{array}$ & $\begin{array}{c}\text { 2-Gate Velocity } \\
(\mathrm{m} / \mathrm{s})\end{array}$ & $\begin{array}{l}\text { Impact } \\
\text { Location }\end{array}$ & Notes \\
\hline 1 (sakplt1) & 5.05 & 20.743 & & $\begin{array}{l}\text { Projectile tilted } \\
\text { slightly nose-up. } \\
\text { Exceedingly slight } \\
\text { bulge on back. }\end{array}$ \\
\hline 2 (sakplt2) & 13.51 & 37.353 & & $\begin{array}{c}\text { Straight flight. } \\
\text { Moderate damage; } \\
2 \text { small cracks on } \\
\text { back } \\
(\sim 0.82 \text {-inches long })\end{array}$ \\
\hline 3 (sakplt3) & 12.53 & 36.157 & & $\begin{array}{c}\text { Straight flight. } \\
\text { Damage on back; } \\
2 \text { small cracks on } \\
\text { back ( 0.51 inches } \\
\text { long). }\end{array}$ \\
\hline 4 (sakplt4) & 11.01 & 34.094 & 8 & $\begin{array}{c}\text { Projectile tilted } \\
\text { slightly nose-up. } \\
\text { Very slight damage } \\
\text { on back; } 2 \text { small } \\
\text { cracks } \\
\text { ( } \sim 0.35 \text { inches long) }\end{array}$ \\
\hline 5 (sakplt5) & 10.01 & 32.259 & & $\begin{array}{c}\text { Projectile tilted } \\
\text { slightly nose-up. } \\
\text { No cracks, but a } \\
\text { small bulge on back } \\
(\sim 0.5 \text { inches long). }\end{array}$ \\
\hline
\end{tabular}




\section{Instrumented Plate Testing}

The final set of impact tests were performed on plates that were instrumented with strain gauges. Each plate was instrumented using two in-plane strain gauge rosettes that record the $\varepsilon_{\mathrm{xx}}, \varepsilon_{\mathrm{yy}}$, and $\varepsilon_{\mathrm{xy}}$ strain time series for subsequent data analysis. Figure II-6 defines the location of the projectile impact and strain gauges. Prior to attaching the strain gauge rosettes and performing the impact tests, a full modal characterization was conducted on each plate, which required four accelerometers and an impact hammer as shown in Figure II-7.

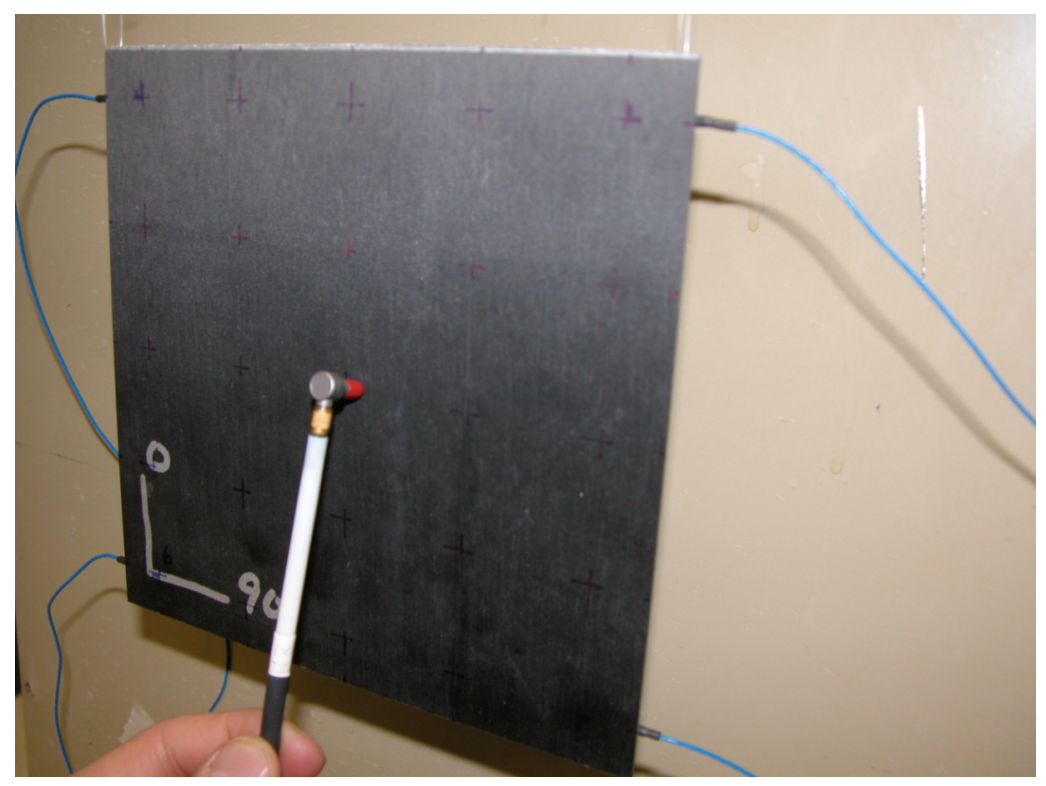

Figure II-7. Instrumentation for modal testing of laminated composite plates.

The gauges used were made by Micro-Measurements; the model number is WA-13-060WR-120. These were in a triaxial (0-, 45-, and 90-degree orientation) stacked rosette with a nominal resistance of 120 Ohms. The strain gauges were bonded to the smooth side of the composite plates using quick-setting M-Bond adhesive. Bondable terminals were attached between the gauge jumper-wires and main lead-wire system to provide a soldering location as well as strain relief loops for the gauge jumper-wires (Figure II-8). Vishay 2311 signal conditioning amplifiers were used to generate high-level signals from the strain gauges and the data were acquired with a Nicolet Odyssey XE data acquisition system at a sample frequency of $500 \mathrm{kHz}$. 


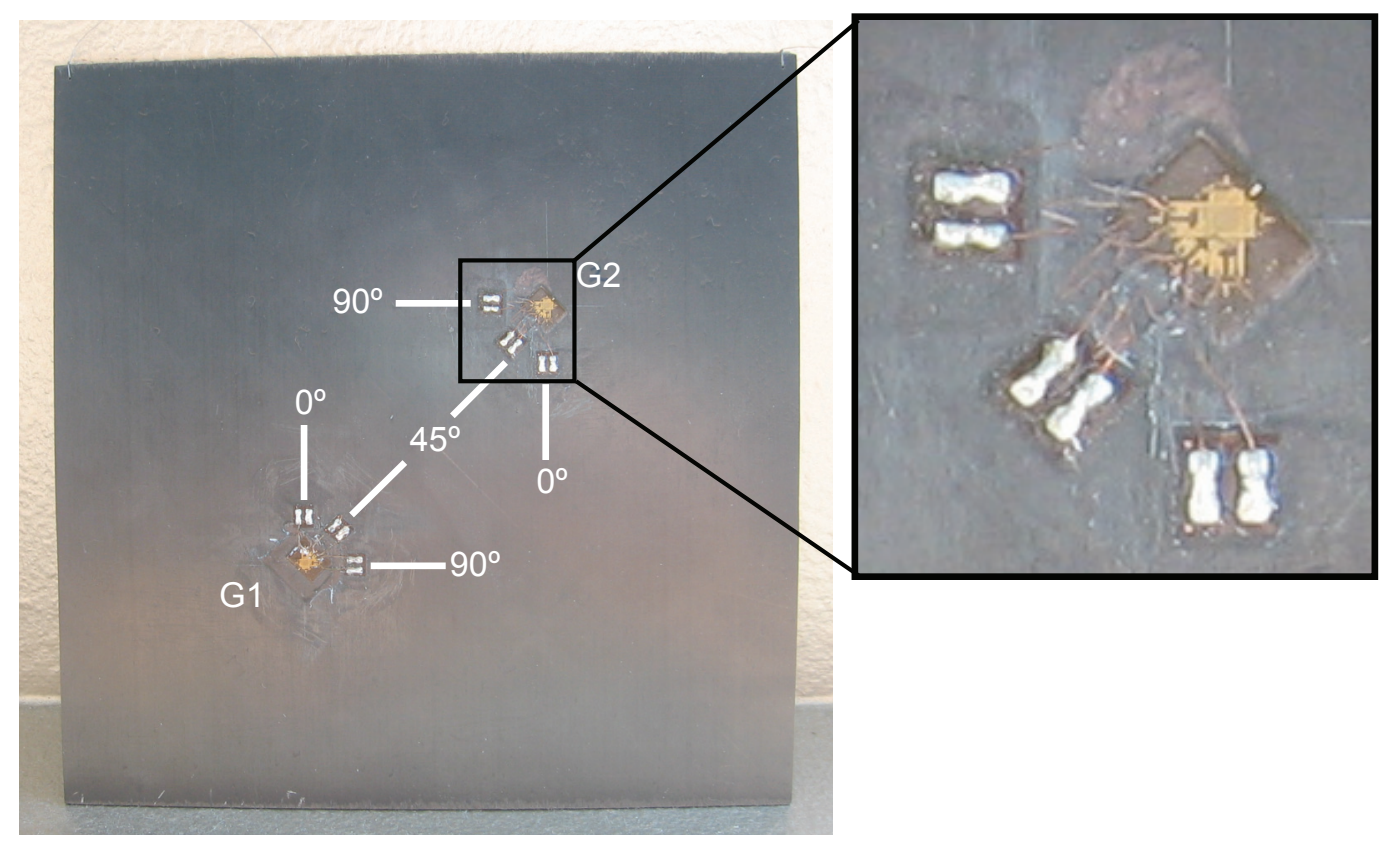

Figure II-8. Strain gauge location and orientation.

The five plates were impacted with the previously used Ti-6Al projectile at velocities ranging from $20.402 \mathrm{~m} / \mathrm{s}$ to $36.286 \mathrm{~m} / \mathrm{s}$; the impacts were performed on the side opposite the strain gauges. During testing, the plate was again suspended inside the steel box using monofilament line attached at each of the upper corners. The high-speed video camera recorded the event from the time that the projectile exited the barrel until after the impact occurred; the frame rate was 22,222 frames per second with a $10 \mathrm{~s}$ exposure at a resolution of 256 by 128 pixels. The intended impact location was the center of the plate and care was taken to ensure that the impact would be orthogonal to the plate's surface. It was important to not introduce excessive amounts of damage because the finite element model, to which the results of the experiments would be compared, was only capable of simulating delamination of the layers and not cracking/breaking of the fiber and/or matrix material. Table II-E provides the operating pressures, impact velocities, impact locations, and a brief description of any visible damage. 
Table II-E. Instrumented plate test summary.

\begin{tabular}{|c|c|c|c|c|}
\hline $\begin{array}{c}\text { Test } \\
\text { (Filename) }\end{array}$ & $\begin{array}{l}\text { Pressure } \\
\text { (psi) }\end{array}$ & $\begin{array}{l}\text { 2-Gate Velocity } \\
(\mathrm{m} / \mathrm{s})\end{array}$ & $\begin{array}{l}\text { Impact } \\
\text { Location }\end{array}$ & Notes \\
\hline 1 (smplt1) & 5.05 & 20.735 & & $\begin{array}{l}\text { Projectile tilted } \\
\text { slightly nose-down. } \\
\text { Exceedingly slight } \\
\text { bulge on back. }\end{array}$ \\
\hline 2 (smplt 2) & 10.04 & 32.357 & & $\begin{array}{l}\text { No video. Very slight } \\
\text { damage on back; } \\
3 \text { small cracks } \\
\text { ( 0.23 inches long). }\end{array}$ \\
\hline 3 (smplt 3) & 12.54 & 36.286 & & $\begin{array}{l}\text { Straight flight. } \\
\text { Damage on back; } \\
2 \text { small cracks } \\
\text { ( 0.74 inches long). }\end{array}$ \\
\hline 4 (smplt 4) & 5.05 & 20.402 & & $\begin{array}{l}\text { Straight flight. } \\
\text { Small bulge on back; } \\
(\sim 0.35 \text { inches long }) \text {. }\end{array}$ \\
\hline 5 (smplt5) & 8.48 & 28.380 & & $\begin{array}{l}\text { Straight flight. } \\
\text { No data obtained. } \\
\text { Small bulge on back; } \\
(\sim 0.40 \text { inches long })\end{array}$ \\
\hline
\end{tabular}

The video results of the instrumented plate tests showed that the strain gauges broke off of the plate shortly after impact (circles, Figure II-9), and although not necessarily a desirable occurrence, it was expected. Additionally, the strain gauge data showed very clean signals for the initial impact, but in the brief amount of time before the gauges came off the plates, there were several instances of shorting between the jumper-wires of the strain gauges. 


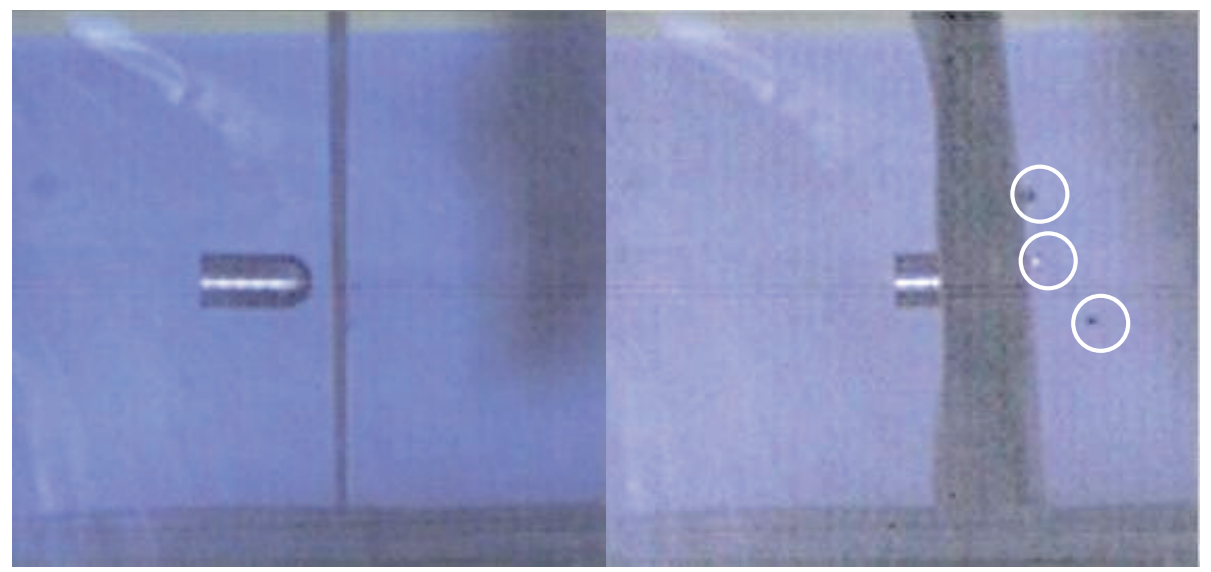

Figure II-9. Plate and projectile before impact (left) and immediately following impact (right).

Figure $\mathrm{II}-10$ is an example plot from test one, gauge two, 0 -degree orientation. The two circles indicate potential jumper-wire shorts; after time $t \approx 12.911$ seconds, the gauge and/or lead-wires have been destroyed by the impact. Enough data were collected to perform the required analysis for correlation to the finite element model; however, in future testing, greater care will be taken to eliminate shorting of the jumper-wires.

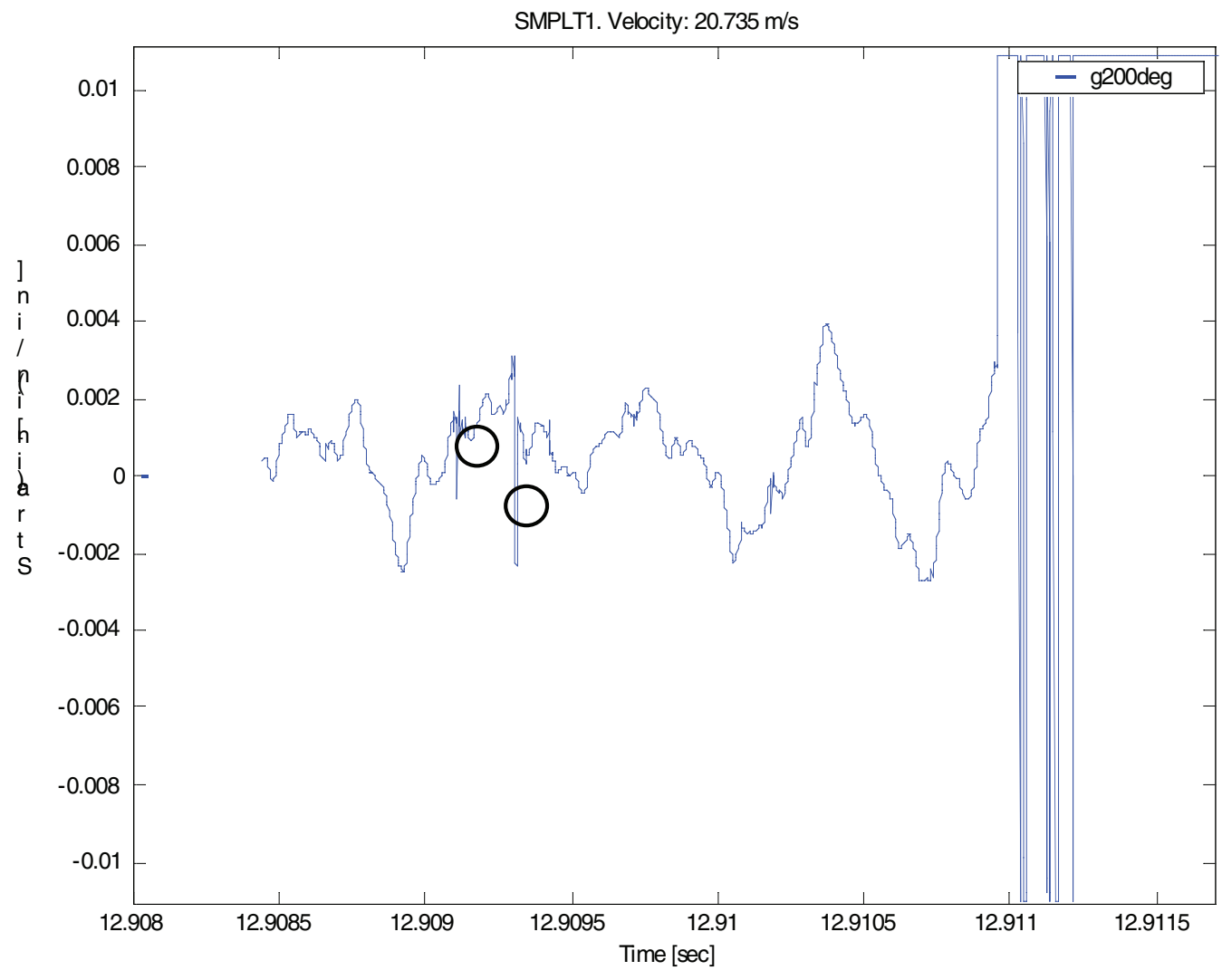

Figure II-10. Plot of strain vs. time for Test One, Gauge 2, 0 degrees. 


\section{Future Comprehensive Test Procedure}

The test matrix shown in Table II-F defines a more comprehensive assessment of the amount of delamination and fiber splitting damage as a function of impact velocity (or energy) in addition to environmental variability. Three levels of impact velocity are needed to fit damageversus-velocity meta-models up to quadratic order. Each impact level is replicated to provide an estimation of testing repeatability.

Table II-F. Future test matrix based on 15 physical experiments.

\begin{tabular}{|l|c|c|c|c|c|}
\hline \multirow{2}{*}{ Impact Velocity $\left(\mathrm{V}_{\mathrm{I}}\right)$} & \multicolumn{5}{|c|}{ Replicate Tests } \\
\cline { 2 - 6 } & 1 & 2 & 3 & 4 & 5 \\
\hline Low Level & Test 1.1 & Test 1.2 & Test 1.3 & Test 1.4 & Test 1.5 \\
\hline Medium Level & Test 2.1 & Test 2.2 & Test 2.3 & Test 2.4 & Test 2.5 \\
\hline High Level & Test 3.1 & Test 3.2 & Test 3.3 & Test 3.4 & Test 3.5 \\
\hline
\end{tabular}

(Numbering convention: The first digit identifies the level of impact velocity, where $1=$ low impact velocity, 2 = medium impact velocity, and $3=$ high impact velocity. The second digit identifies the replicate number, with up to five replicates for each level of impact velocity.)

\section{Testing Procedure}

For each of the 15 plates:

1. Drill holes in two corners of the plate, as shown in Figure II-6, for the supporting monofilament. The holes are centered at $3.2 \mathrm{~mm}$ (1/8 inches) from each edge and are $0.5 \mathrm{~mm}$ (0.02 inches) in diameter.

2. Conduct a modal characterization of the plate to verify the resonant frequencies prior to impact testing. Table II-G lists statistics obtained from testing eight similar plates with free-free boundary conditions. If the measured frequencies deviate from the values listed in Table II-G by more that 2 standard deviations, the plate is considered defective and is disregarded.

3. Attach two strain gauge rosettes with orientations $[0 ; 90 ; 45]$ degrees to the smooth side of the plates as shown in Figure II-6.

4. Suspend the plate with monofilament and orient the plate so that the smooth side is facing the gas gun. 
5. Shoot the plate with the aluminum projectile in accordance with the test matrix defined in Table II-E, impacting the plate at its approximate center. Measure the response of the two strain gauge rosettes and the impact velocity. Also note any particular observations that are pertinent, e.g., sensors damaged during the impact, breaking of the monofilament supports, etc.

6. Analyze the plate after the impact using ultrasonic imaging to observe any delamination or fiber splitting that is present.

Table II-G. Statistics of identified modal frequencies obtained with eight composite plates.

\begin{tabular}{|c|c|c|c|}
\hline Mode Number & Mean Frequency & Standard Deviation & Relative Deviation \\
\hline 1 & 107.37 Hertz & 1.05 Hertz & $0.98 \%$ \\
\hline 2 & 191.81 Hertz & 2.37 Hertz & $1.24 \%$ \\
\hline 3 & 274.06 Hertz & 2.92 Hertz & $1.07 \%$ \\
\hline 4 & 315.31 Hertz & 3.13 Hertz & $0.99 \%$ \\
\hline 5 & 398.88 Hertz & 3.00 Hertz & $0.75 \%$ \\
\hline
\end{tabular}

(The relative deviation is the standard deviation divided by the mean, expressed in percent.)

\section{Summary}

The procedure used for experimental testing provided consistent results with which to make good comparisons. We have been able to identify three small ranges of velocity impact values that give the desired damage levels. These damage levels can subsequently be used to compare with the finite elements simulations. Part of the reason that small ranges of values were identified was because of the stringent testing operation. It's true that there still remains unaccounted for variability such as in the location of impact, but with a test matrix as in Table II-F, we are optimistic that we can isolate the uncertainty to replicable intervals of values for damage area. This will be very useful to improve on the composite plate finite element model and the parameter uncertainty using GIT. 


\section{Chapter III. Modeling and Simulation}

Composite laminates offer many advantages to structural designers, including low density, high strength, and high stiffness. They also present challenges in the form of complex failure modes that occur on various length scales and that often interact with each other. One of goals of the Damage Prognosis project at Los Alamos National Laboratory is to develop an advanced composite modeling capability to meet this challenge.

In order to create a model with sufficient accuracy to predict damage evolution, it is important to model the structure at all length scales at which damage and other forms of nonlinearity may occur. For laminated fiber composites, there are at least three length scales that must be considered: the macroscale, associated with the in-plane plate dimensions; the mesoscale, associated with the ply thickness; and the microscale, or material length scale, associated with the fiber diameter.

At the microscale, it is necessary to model visco-elasticity and plasticity in the polymer matrix, debonding between the fiber and matrix, fiber fracture, and matrix fracture to capture the possible material nonlinearities of the composite. All of these phenomena evolve in a highly nonlinear and history-dependent fashion. Some of these processes are also of a stochastic nature, as is the microstructure itself, which further complicates analysis at this length scale. A linear material model based on homogenization is currently used to simplify the study of higher-scale nonlinearities. However, colleagues at LANL are developing a versatile and computationally efficient material model in parallel based on a new stochastic transformation field analysis (STFA) (see Williams and Tippetts [3] and Williams [4]). When integrated with the higher-scale finite element model, this material model will provide the capability to correctly predict the probabilistic, nonlinear response of both the constituent phases and the bulk composite material.

At both the macroscale and mesoscale, the damage mode of interest for impact damage is fracture. The damage modes of interest in this study, because of their effect on subsequent structural performance, are ply splits and delamination. Ply splits are a type of fracture through the ply thickness and parallel to the fibers and occur as combinations of matrix fracture and fiber/matrix debonding. Delamination is an area of disbond between adjacent plies. When a plate is subjected to transverse impact, experiments have shown that damage initiates as a ply split through the outermost ply opposite the impact side as explained in Choi and Chang [5]. 
This ply split propagates laterally and initiates a delamination between the outermost ply and the one adjacent to it. The purpose of this work is to accurately model this type of damage and verify the model with the help of experimental results (Chapter II) and Generalized Information Theory (GIT) (Chapter IV).

\section{Finite Element Model}

A finite element model was developed to simulate impact damage in composite plates. The plate has dimensions of 6 inches by 6 inches, with eight plies of 0.005 inches thickness. The plies are a unidirectional graphite/epoxy fiber composite material, with a symmetric lay-up of $[0 /+45 / 90 /-45]$ s. That is, relative to the top ply, the fiber orientations of the plies in descending order are 45, 90, -45, -45, 90, 45, and 0 degrees. The orthotropic material properties are listed in Table III-A. The plate is meshed with 20-node quadratic hexahedra, with ten elements in each in-plane direction and one element through the thickness of each ply.

Table III-A. Composite material properties.

\begin{tabular}{|c|c|}
\hline Material Property & Value \\
\hline$E_{11}$ & $132.4 \mathrm{GPa}$ \\
\hline $\mathrm{E}_{22}$ & $9.136 \mathrm{GPa}$ \\
\hline $\mathrm{E}_{33}$ & $9.136 \mathrm{GPa}$ \\
\hline$v_{12}$ & 0.3000 \\
\hline$v_{13}$ & 0.3000 \\
\hline$v_{23}$ & 0.4000 \\
\hline $\mathrm{G}_{12}$ & $4.548 \mathrm{GPa}$ \\
\hline $\mathrm{G}_{13}$ & $4.548 \mathrm{GPa}$ \\
\hline $\mathrm{G}_{23}$ & $3.000 \mathrm{GPa}$ \\
\hline Density, $\rho$ & $1522 \mathrm{~kg} / \mathrm{m}^{3}$ \\
\hline
\end{tabular}


The projectile was a $32 \mathrm{~mm}$ long, $1.5 \mathrm{~cm}$ diameter hemispherically-tipped cylinder that impacted the plate (see Figure II-3). The isotropic material properties for the Ti-6Al projectile are listed in Table III-B.

Table III-B. Projectile material properties.

\begin{tabular}{|c|c|}
\hline Material Property & Value \\
\hline$E$ & $200.0 \mathrm{GPa}$ \\
\hline$v$ & 0.3333 \\
\hline Density, $\rho$ & $7850 \mathrm{~kg} / \mathrm{m}^{3}$ \\
\hline
\end{tabular}

The projectile was meshed with 10-node quadratic tetrahedral elements. The meshes for both the projectile and plate are shown in Figure III-1.

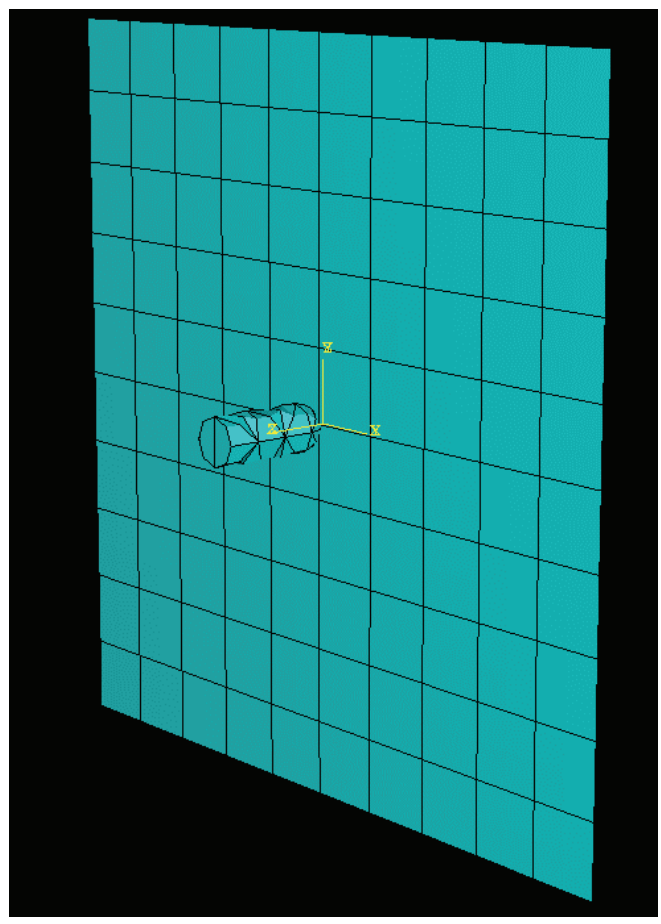

Figure III-1. The projectile is meshed with quadratic tetrahedral elements, and the laminate plate is meshed with quadratic hexahedra elements.

The ply split and delamination fracture surfaces are modeled with special elements that use a Cohesive Zone Model (CZM) as the constitutive behavior. The constitutive model for the CZM is described in the next section. Figure III-2 shows the CZM elements for the ply split and delamination viewed from the edge of the plate. 


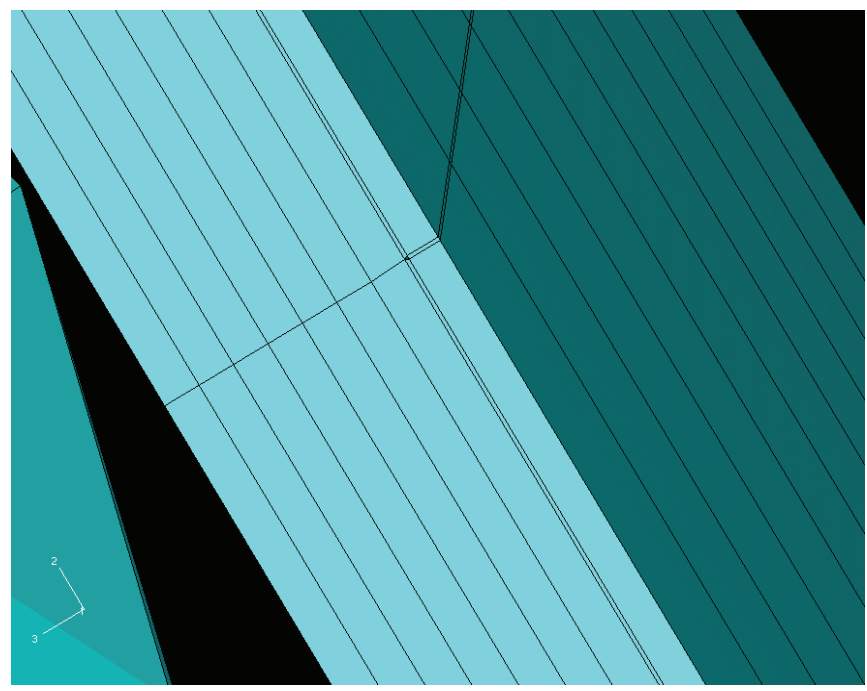

(a)

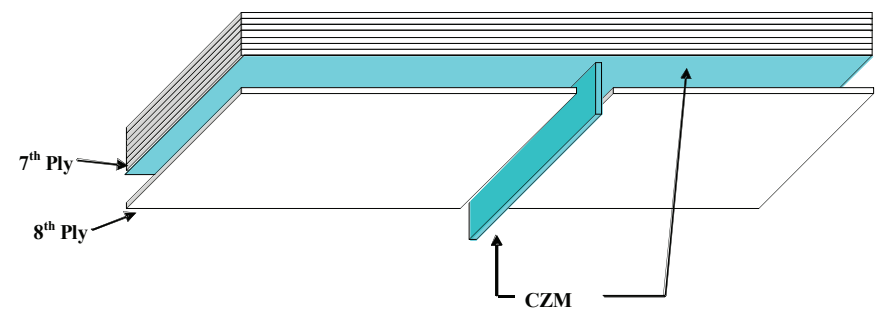

(b)

Figure III-2. (a) Close-up of the laminate plies showing the CZM elements. (b) Schematic of CZM elements and their location relative to the plies.

The finite element model was implemented with the ABAQUS software suite. A user element for the CZM surfaces was defined using the UEL user subroutine interface. An ABAQUS python interface script was written to access the output database for visualization of the fracture surfaces.

Because the quickly evolving damage in the plate impact simulation requires a very small time step, a formulation that is explicitly integrated in time could be most efficient. However, ABAQUS has the limitation of only allowing user-defined elements in implicit simulations. Therefore, an implicit solver was used for the results in this study.

\section{Cohesive Zone Model (CZM)}

A Cohesive Zone Model (CZM) defines an interfacial traction vector, $T$, as a function of the relative displacement (or displacement jump) vector, $u$, at a point on the two interface surfaces. A simple function with a few parameters is usually chosen for $T(u)$. Many CZMs have the general form

$$
T(u)=E_{i} v F(\lambda)
$$


where $E_{i}$ is the initial stiffness of the interface, and $v=\frac{u}{\delta}$ is the relative displacement normalized by the critical displacement jump $\delta, \lambda$ is the parameter that quantifies the amount of damage, and $F(\lambda)$ is a damage function. The parameters $E_{i}$ and $\delta$ may be unique for each component of $v$ and $T$, which allows for the possibility of mode-dependent fracture properties.

Fracture is usually discussed in the context of three distinct modes. In mode I, fracture occurs in a tensile opening motion where the relative displacement across the fracture surface is orthogonal to that surface. Mode II is a shear sliding mode, in which the relative displacement is in the plane of fracture and in the direction of crack front propagation. Mode III is a shear tearing mode, in which the relative displacement is in the plane of fracture and orthogonal to the direction of crack front propagation. CZMs may be defined to handle the three modes of fracture simultaneously.

The traction vector may be physically interpreted as the traction on the fracture interface that holds the two surfaces together. An undamaged interface with small (yet finite) thickness should have a traction vector that is a linear function of the displacement jump across the interface. A fractured interface should have zero traction on the two surfaces, unless the fracture surface is closed in compression. In the case of a perfectly sharp crack, a point on the crack surface would experience an instantaneous transition between these two states as the crack front passed through it. However, even very brittle materials cannot sustain the infinite stresses that a perfectly sharp crack would cause near the crack tip. Real materials exhibit highly nonlinear local damage modes such as plasticity and ductile tearing, crazing and void formation, and microcracking. Physical processes like these evolve as the interfacial tractions vary continuously from the linear, undamaged state to the traction-free fractured state. By including a continuous transition from an undamaged to a fractured surface, CZMs represent these complex local damage processes while also mitigating the numerical problems caused by discontinuous changes in the stress state.

The damage function $F(\lambda)$ can be defined in many ways, but is usually a continuous function of $\lambda$ varying between 0 and 1 with the properties

$$
\begin{aligned}
& F(0)=1 \quad \text { undamaged, pristine state } \\
& F(1)=0 \quad \text { complete separation }
\end{aligned}
$$


A simple example for the damage function $F(\lambda)$ is

$$
F(\lambda)= \begin{cases}(1-\lambda)^{2} & \lambda \leq 1 \\ 0 & \lambda>1\end{cases}
$$

In monotonic mode I fracture, Equation (III-3) is equivalent to both the Chaboche [6] and Tvergaard [7] CZMs.

The parameter $\lambda$ is often defined as the maximum magnitude of the vector $v$ if the mode I component is nonnegative, and as the maximum magnitude of the mode II and III components of $v$ if the mode I component is negative. When the crack cannot heal, i.e., the fracture process is irreversible, the damage parameter $\lambda$ must increase monotonically to account for the fracture history. A typical CZM response curve is given in Figure III-3.

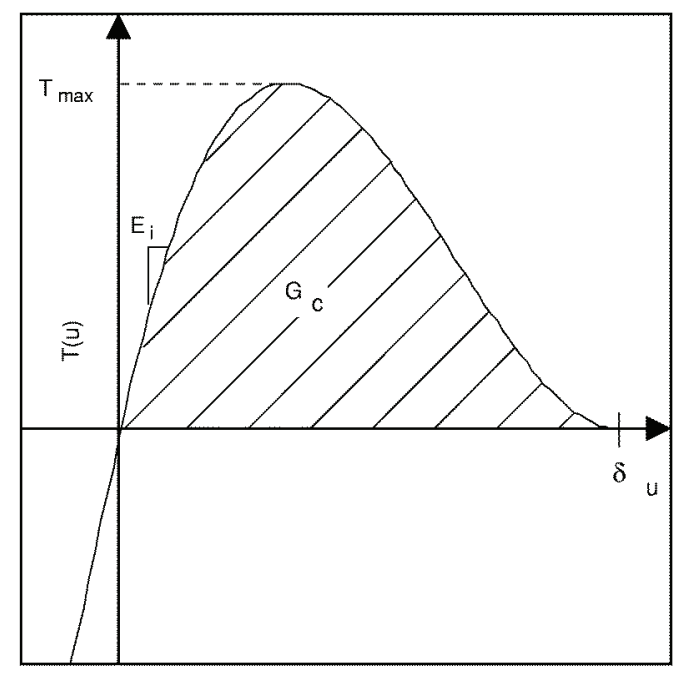

Figure III-3. Quadratic damage CZM in mode I fracture.

If the relative displacement across the interface is negative, the interface has a linear stiffness to penalize interpenetration. As the positive relative displacement increases from zero, initially the response is nearly linear and the interface is undamaged but as loading continues the response transitions to a nonlinear behavior indicating the onset of damage. This process continues until the interfacial traction reaches a maximum, $T_{\max }$. After this point the interface response softens. When the displacement jump reaches the critical distance $\delta$, the interfacial traction becomes zero and the interface is considered to be completely separated. At this point, the energy consumed by the interface per area is $G_{c}$, illustrated in Figure III-3 as the area under the irreversible part of the $T(u)$ curve. 
In the finite element implementation of the CZM, it was necessary to evaluate surface integrals which include Equation (III-1) or its derivatives with respect to $u$ in the integrand. As with other integrals encountered in finite element formulations, the complexity of the expressions makes analytical integration practically impossible. Numerical integration algorithms are employed which can give accurate results if the integrand is well-approximated by a low-order polynomial. For CZMs of the form of Equations (III-1) and (III-3), however, there are discontinuities in the derivatives that make them problematic to integrate numerically. An improved algorithm, based on adaptive division of the integration domain (Genz and Malik [8], Van Dooren and De Ridder [9]), solves this problem and facilitates the use of these CZMs (Tippetts et al. [10]).

Four different parameters are identified in Figure III-3 and the preceding discussion. However, only two of these, for each mode, are independent. It is also important to note that two

of these fracture parameters $\left(T_{\max }\right.$ and $G_{c}$ ) are associated with the macroscale. The fact that the CZM requires only two macroscale parameters makes experimental measurement and validation very convenient compared to other fracture models, many of which include a number of parameters that must be measured at the microscale. The experimental results will help to validate the values of the parameters for the composite material used in this model.

\section{Impact Damage Results}

This section describes preliminary work conducted by Tippetts and Hemez in [1] and serves as the basis upon which this current study builds. As in this current study, the finite element model was executed with no displacement constraints (free boundary conditions). However Tippetts and Hemez [1] used an initial velocity of $50 \mathrm{~m} / \mathrm{s}$ for the projectile for validation as opposed to our parametric study that uses three velocities: $10 \mathrm{~m} / \mathrm{s}, 25 \mathrm{~m} / \mathrm{s}$, and $40 \mathrm{~m} / \mathrm{s}$. They state in [1] that as shear waves propagate out from the point of impact, the plate experiences a bending deformation as shown in Figure III-4 (a). Initially, this was in good qualitative agreement with impact experiments in [1]. For example, Figure III-4 (b) shows an image from a similar plate impact experiment just after projectile contact. 


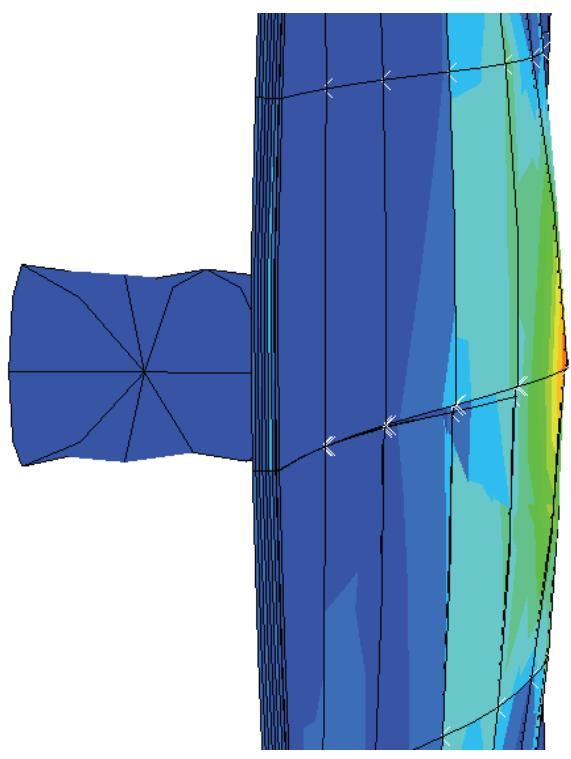

(a)

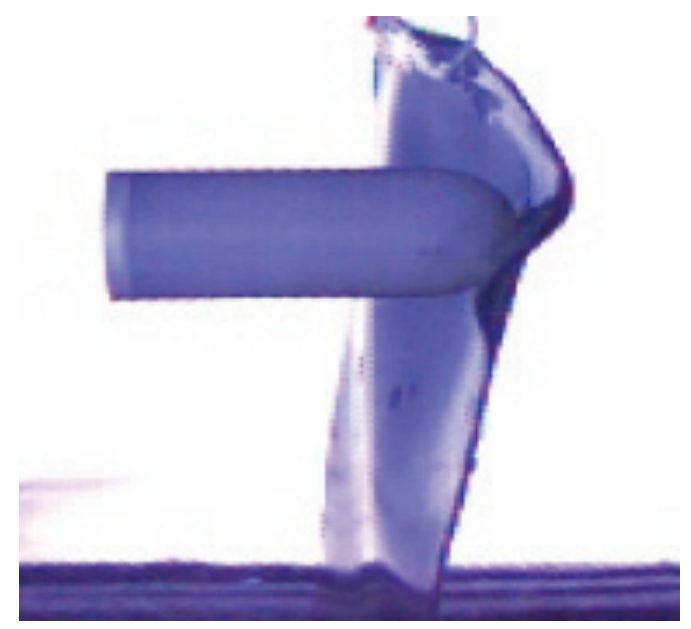

(b)

Figure III-4. (a) Simulation result of impact producing large bending displacements (contours are Mises stress). (b) Snapshot from a composite plate impact experiment showing large bending deformation.

Shortly after the bending deformation loads the CZM elements on the back of the plate, a ply split opens and propagates to each side. This ply split is shown in Figure III-5.

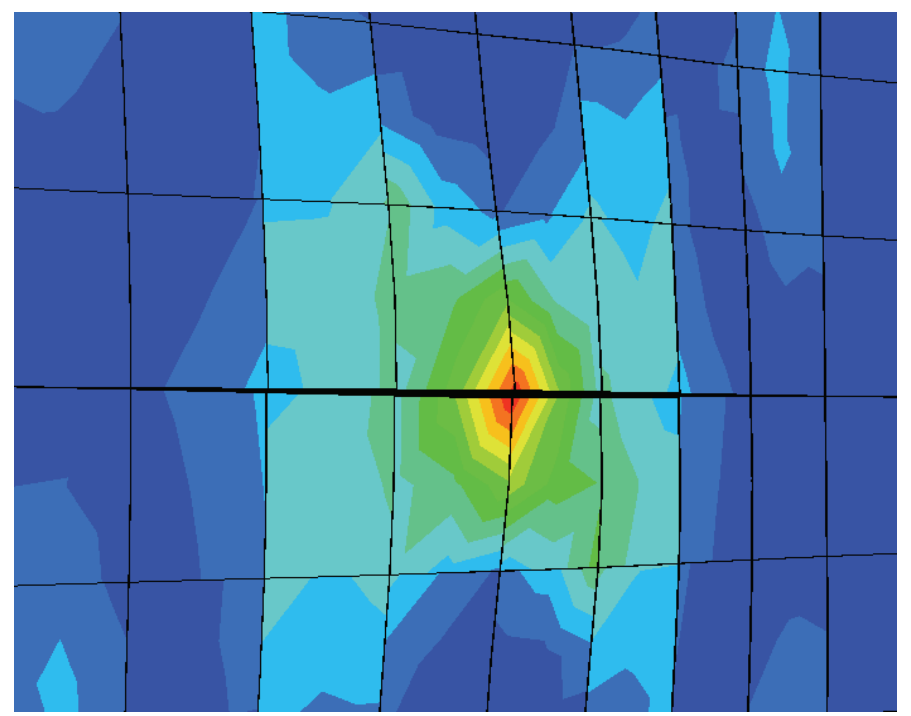

Figure III-5. The ply split on the surface opposite impact is captured by the fracture model. The contours are of Mises stress. 
As the ply split grows and the plate continues to bend, a delamination forms under the split and grows out from the center of impact. Figure III-6 shows the delamination with contours of $\lambda$, the CZM damage parameter.

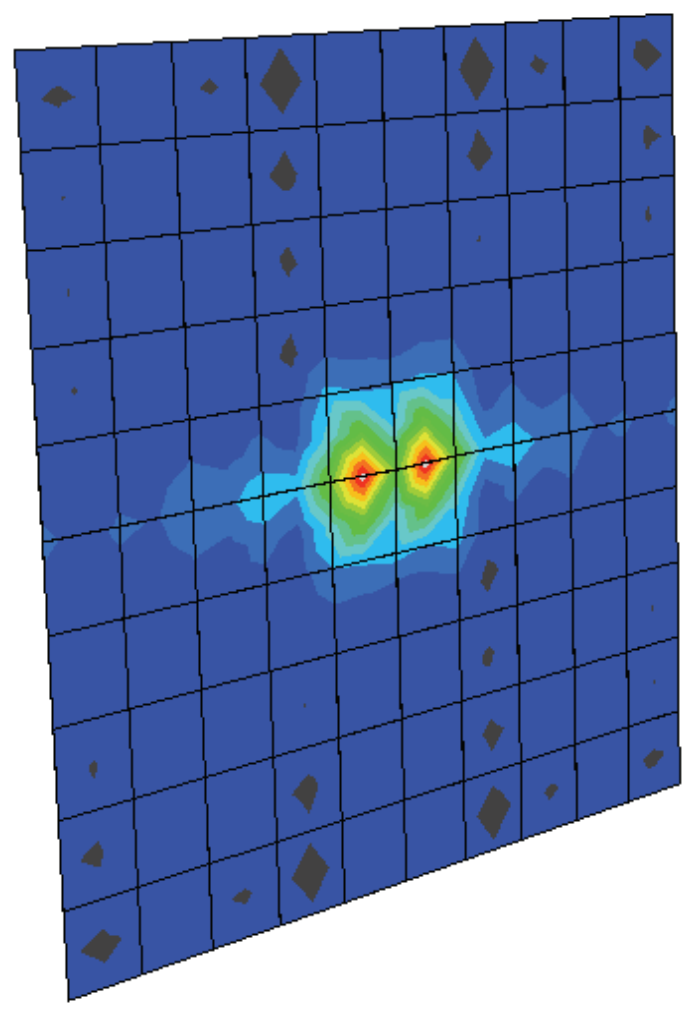

Figure III-6. A delamination forms between the last two plies opposite the impact location, oriented parallel to the ply split. The contours are of $\lambda$, the damage parameter.

The qualitative observations of both the progression and interaction between these two damage modes were consistent with experiment. An ultrasound C-scan image of post-impact delaminations in a composite laminate is shown in Figure III-7.

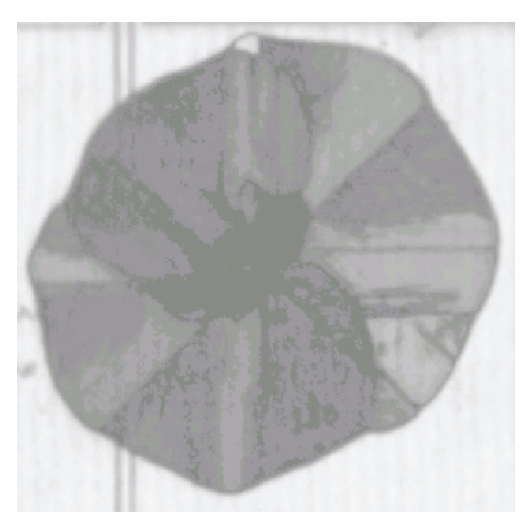

Figure III-7. Ultrasound C-scan shows delaminations caused by impact. 
The plate used for the impact experiment of Figure III-7 was made from the same composite material as the simulations, but with more plies in the layup (Nadler et al. [11]). Therefore, multiple elongated delaminations similar to the one shown in Figure III-6 are visible in Figure III-7, stacked at various ply interfaces in four different orientations.

Tippetts and Hemez [1] discovered that a crack is only possible where a CZM element already exists. As such it is necessary to include CZM elements at all surfaces where a crack will be allowed to propagate. This is perhaps one of the greatest limitations of this type of CZM. This finding is confirmed in this study by first conducting a parametric study that uses the same CZM configuration. Subsequently we modify this configuration to include the CZM through all the layers.

\section{Parametric Study}

A parametric study was performed to validate the values of the model parameters, namely the maximum interfacial traction $\left(T_{\max }\right)$, the energy per area $\left(G_{c}\right)$, the hertz contact stiffness $\left(k_{n l}\right)$, and the projectile velocity. The purpose of this study was to vary a few key parameters to allow uncertainty in the final delamination quantities. Consequently, the dispersion of delamination values would be used in the various GIT theories as a means to quantify uncertainty and show the utility of the various GIT theories. The values of the parameters are shown in Table III-C. High and Low values are used for energy $\left(G_{c}\right)$, maximum stress $(\sigma)$, and hertz contact stiffness $\left(k_{n l}\right)$. The reason that we used only the two values for each of these three parameters is because we want to pay particular attention to the final output delamination values which will be used in the GIT methods. And using two values will help us determine if we are enclosing the actual parameter values. As for the velocity, however, we used three values, $(10,25$, and 40$) \mathrm{m} / \mathrm{s}$. We used these three values to approximate the three levels of experimental damage as in Chapter II. 
Table III-C. Parametric study values.

\begin{tabular}{|c|c|c|c|c|}
\hline Run \# & $\operatorname{Gc}\left(\mathrm{J} / \mathrm{m}^{2}\right)$ & $\sigma\left(\mathrm{N} / \mathrm{m}^{2}\right)$ & $k_{n l}$ & $\begin{array}{c}\text { Velocity } \\
(\mathrm{m} / \mathrm{s})\end{array}$ \\
\hline 1 & 200 & $3.41 \mathrm{E}+07$ & 1 & 10 \\
\hline 2 & 200 & $3.41 \mathrm{E}+07$ & 1 & 25 \\
\hline 3 & 200 & $3.41 E+07$ & 1 & 40 \\
\hline 4 & 590 & $3.41 \mathrm{E}+07$ & 1 & 10 \\
\hline 5 & 590 & $3.41 \mathrm{E}+07$ & 1 & 25 \\
\hline 6 & 590 & $3.41 \mathrm{E}+07$ & 1 & 40 \\
\hline 7 & 200 & $4.25 \mathrm{E}+07$ & 1 & 10 \\
\hline 8 & 200 & $4.25 E+07$ & 1 & 25 \\
\hline 9 & 200 & $4.25 E+07$ & 1 & 40 \\
\hline 10 & 590 & $4.25 E+07$ & 1 & 10 \\
\hline 11 & 590 & $4.25 E+07$ & 1 & 25 \\
\hline 12 & 590 & $4.25 E+07$ & 1 & 40 \\
\hline 13 & 200 & $3.41 \mathrm{E}+07$ & 2 & 10 \\
\hline 14 & 200 & $3.41 \mathrm{E}+07$ & 2 & 25 \\
\hline 15 & 200 & $3.41 \mathrm{E}+07$ & 2 & 40 \\
\hline 16 & 590 & $3.41 \mathrm{E}+07$ & 2 & 10 \\
\hline 17 & 590 & $3.41 \mathrm{E}+07$ & 2 & 25 \\
\hline 18 & 590 & $3.41 \mathrm{E}+07$ & 2 & 40 \\
\hline 19 & 200 & $4.25 E+07$ & 2 & 10 \\
\hline 20 & 200 & $4.25 E+07$ & 2 & 25 \\
\hline 21 & 200 & $4.25 E+07$ & 2 & 40 \\
\hline 22 & 590 & $4.25 E+07$ & 2 & 10 \\
\hline 23 & 590 & $4.25 E+07$ & 2 & 25 \\
\hline 24 & 590 & $4.25 E+07$ & 2 & 40 \\
\hline
\end{tabular}

Figures III-8 (a) and (b) show the resulting damage contour plots for runs 1 and 3 of the parametric study. The gray color represents complete separation or delamination $(\lambda=1)$. Figure III-8 (a) is a low velocity shot and as can be seen delamination begins from the edges of the plate. Figure III-8 (b), a high velocity shot, shows the damage zone extending from edge to edge across the composite plate. This rectangular damage zone is similar for all medium and high velocity parametric runs and coincides with the CZM ply split fracture surface. The damage 
extends across the plate from edge to edge and is different from the previous study in [1] and the scanned image of Figure III-7. The reason for this difference in simulation results is that in [1] the plate results are damage initiation. The damage initiation agrees with the scanned image of Figure III-7. However, if the simulation was allowed to progress, then the same edge to edge damage zone would be present. Figure III-8 (b) is non-circular and suggests a bias for the plysplitting modeled by the CZM between fibers of the last ply as in Figure III-2. Furthermore, Figure III-8 doesn't agree with the scanned images from experiment in this present study.

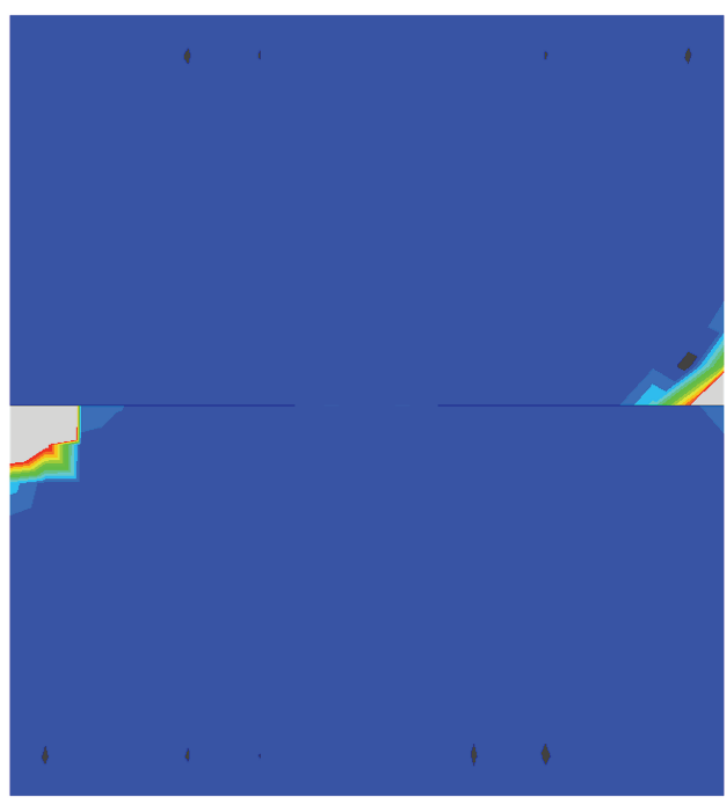

(a)

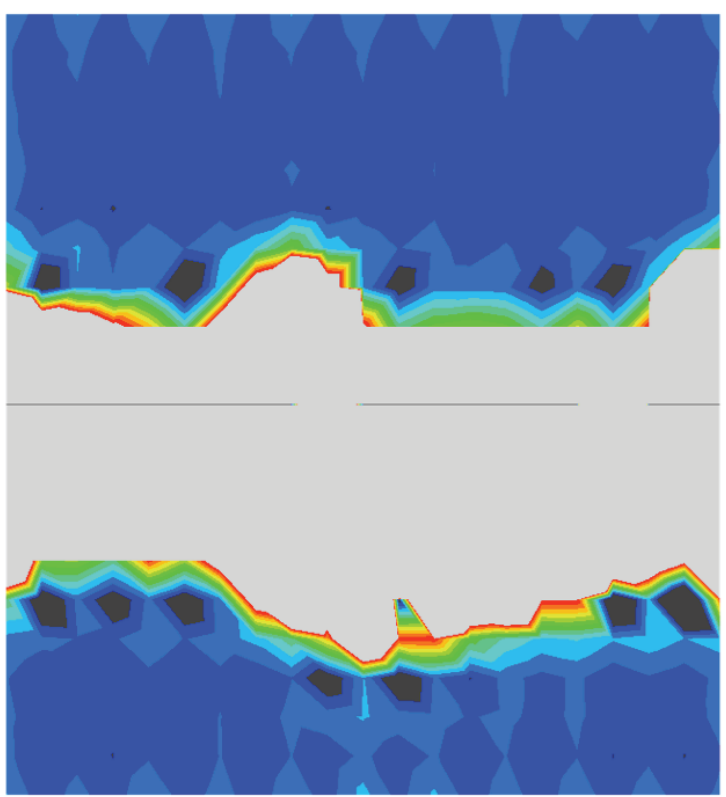

(b)

Figure III-8. (a) FEM model results for parametric study run \#1. (b) FEM model results for parametric study run \#3 (delamination area shown in gray).

Ultrasonic scans are shown in Figures III-9 (a) and (b) for low and high velocities shots, respectively. The ultrasonic scans show delamination roughly in the center of the image. The two areas off center are the strain gauges. As can be seen, the FEM model doesn't capture the shape of the delamination area. The projectile impact velocities on the experimental plates were lower than that in [1] and resulted in smaller damage areas. In spite of this difference, the shapes are similarly circular-like and not edge to edge as in this FEM model. 


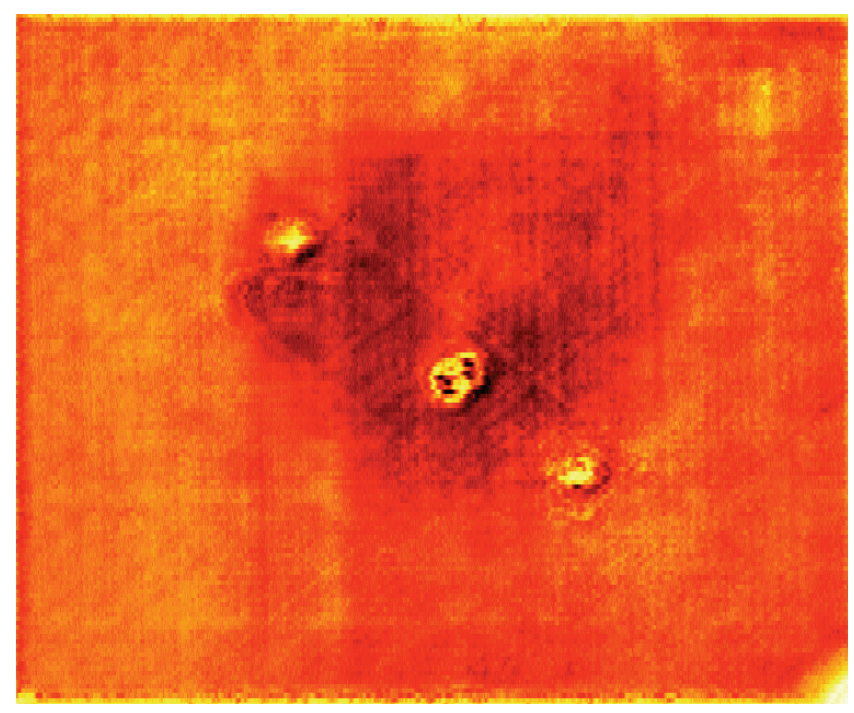

(a)

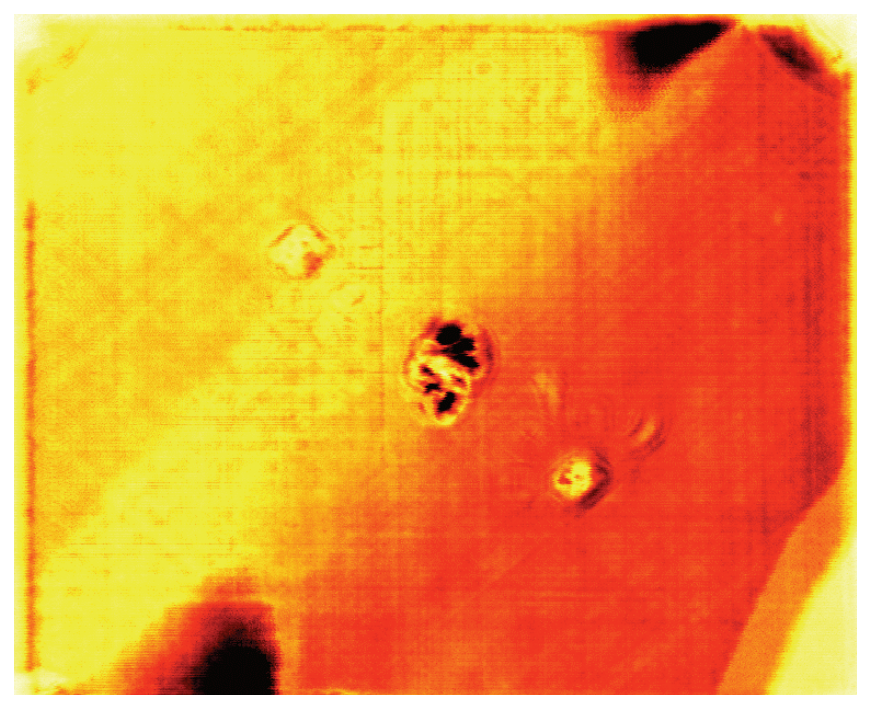

(b)

Figure III-9. (a) Ultrasonic scan of damaged area for velocity= $20.402 \mathrm{~m} / \mathrm{s}$. (b) Ultrasonic scan for velocity $=32.357 \mathrm{~m} / \mathrm{s}$.

In order to capture the observed experimental delamination shape in the FEM model, the composite plate model was partitioned in the center to an area of about 1.44 inches or 2 elements by 2 elements. In this partitioned area, CZM delamination elements were added between each ply (see Figure III-10). The reason we did this is because in the previous version of the FEM model the transmitted energy through the composite plate thickness may have been constrained to the in-plane CZM delamination fracture surface of the last ply; so adding the CZM elements between each ply won't constrain the energy to just the last ply. The results of this new composite plate structure are shown in Figure III-11. The delamination area is now similar in shape to the ultrasonic scans. The model also shows delamination at the corners of the plate. 


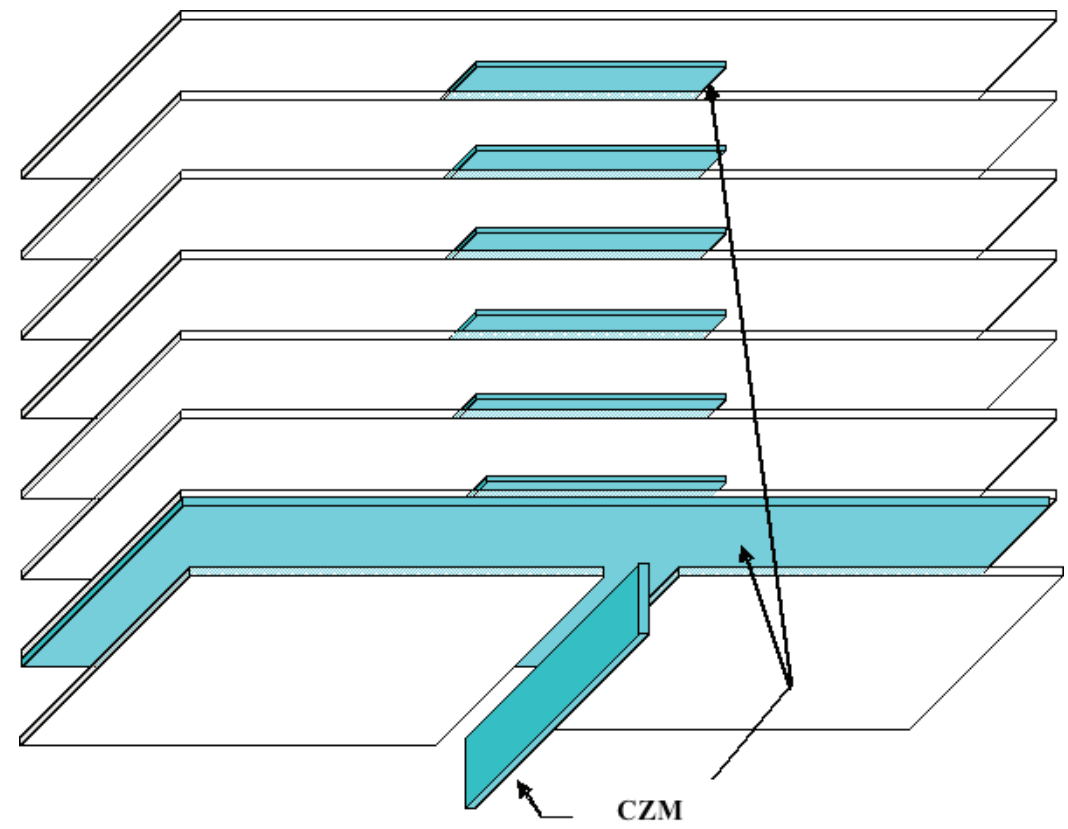

Figure III-10. Schematic of the redesigned FEM composite plate model.

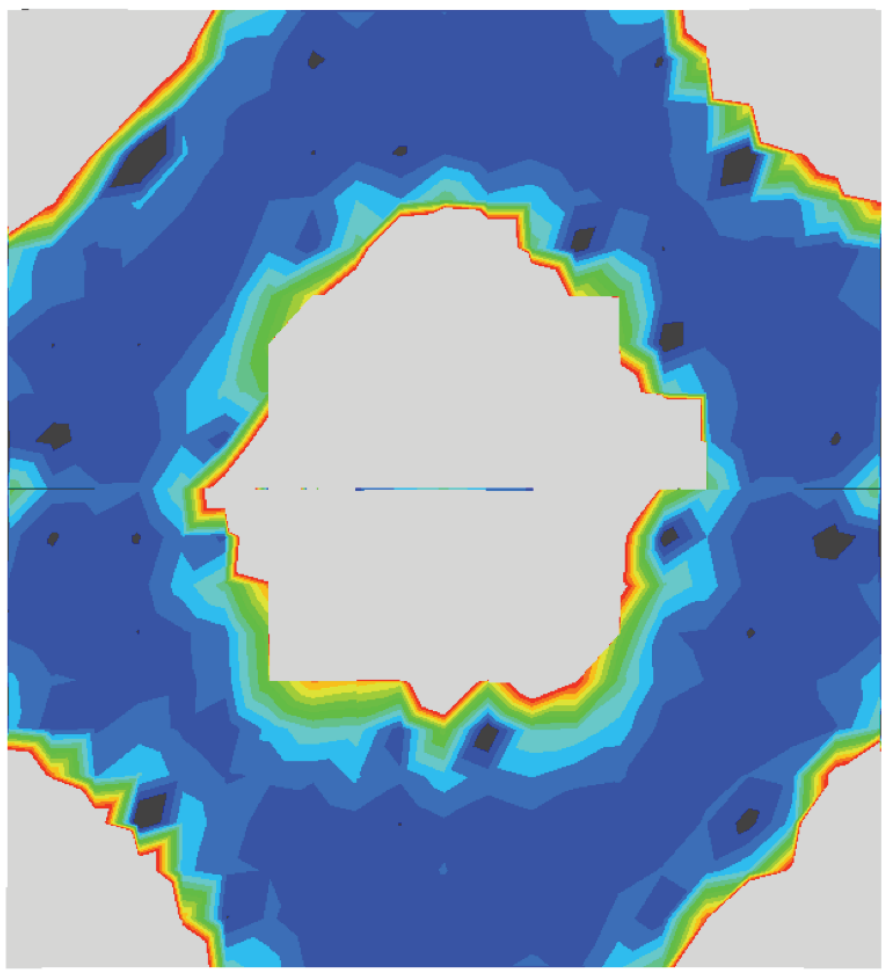

Figure III-11. Results of the redesigned FEM composite plate model results (delamination area in gray). 


\section{Summary}

Clearly the redesigned FEM composite plate model needs work. The area of delamination is much larger than the areas seen in the scanned images from the experiments. However, this new design does point us in the right direction. It shows us that allowing CZM energy "dissipation" throughout the thickness of the plate gives an unbiased damage area that resembles that from experimental impact. One of the pressing needs in the developing field of structural damage prognosis is that of physics based models for simulating the initiation and evolution of damage in advanced materials. Damage initiation caused by transverse impact on composite laminates was modeled adequately in [1]. We now build on that study to model damage evolution more accurately. The model has been implemented in a finite element formulation and has demonstrated the capability of predicting similar damage modes observed in plate impact experiments. 
This page is left blank intentionally. 


\section{Chapter IV. Uncertainty Quantification}

Generalized Information Theory (GIT) defines a class of methods that incorporate evidence which is less constrained by the axioms of probability theory, but which includes probability theory as a special case. Why change what isn't broken? Do statistical analysis, sensitivity analysis, Monte Carlo simulation and probability theory work appropriately for every engineering problem? A new class of methods is available that addresses real-world engineering problems by accommodating sparse data and uncertainties such as measurement errors, random fluctuations, lack of information, and model defects. Pierre Duhem, a $19^{\text {th }}$ century physicist, said, "Experimental verifications are not the base of the theory but its crown" [12]. Similarly, Karl Popper an early $20^{\text {th }}$ century physicist refuted the use of induction and argued against the traditional use of probability theory for scientific research [13]. Their work and the work of subsequent followers portended a wave of research towards less constrictive ways to include evidence, account for uncertainty, and portray systems (or decisions) more realistically, while subsuming probability theory.

Composite plate delamination testing and prediction epitomize the difficulties of model development and system prediction with sparse data and manifold uncertainties. The predominant uncertainty quantification theory in most engineering data analysis is probability theory. However, probability theory comes with many assumptions that belie the real world situation. The objective of this research is to supplement existing probability tools to quantify uncertainty contained within a variety of sources and with multiple definitions.

This research has three components: image analysis, experimental testing, and Finite Element (FE) simulation. Image analysis synthesizes the uncertainties due to both test and FE simulation results. Yet, the image analysis itself has inherent uncertainties. Table IV-A is a summary of these three component uncertainty sources categorized into four definitions: ambiguity, randomness, nonspecificity, and interpretation. Ambiguity is due to insufficient knowledge which can be reduced given ample resources. Randomness represents the uncertainty associated with unavoidable behavior (and the inability to duplicate it); it is irreducible and represents the natural instability of information within certain bounds. Nonspecificity is general information about a class of alternatives, rather than specific evidence on individual alternatives. Finally, interpretation is subjective and represents more of a contextual uncertainty. Interpretation is prevalent in assessing damage severity, the onset of delamination, and even the model used for FE simulation. Clearly, these uncertainties 
(as distinct as they are) still overlap. As such, forcing these uncertainties into the constraints of probability theory as random uncertainty is not an accurate acknowledgement of system uncertainty and would not be a direct effort towards validation.

Table IV-A. Sources of uncertainty categorized into four types.

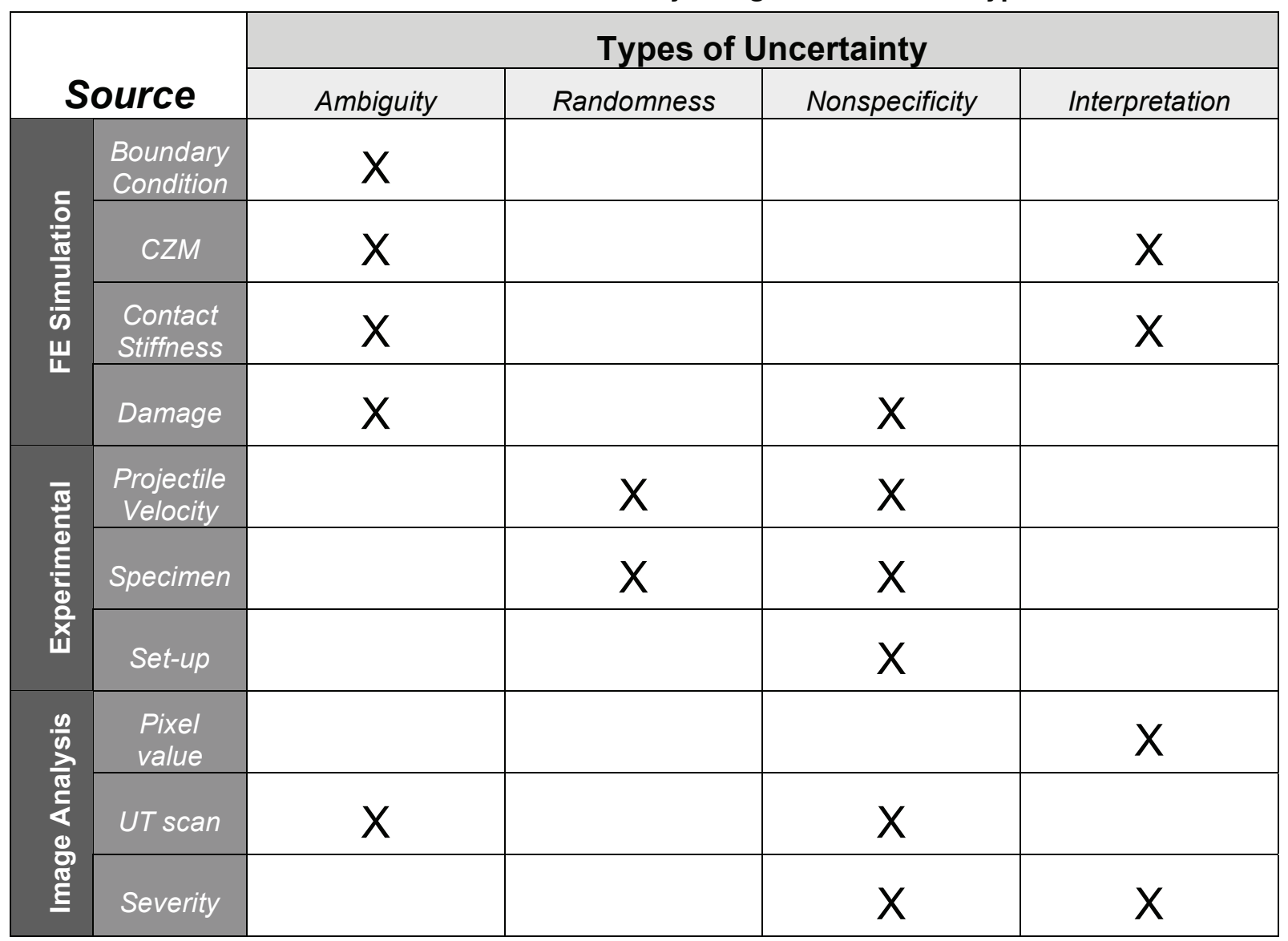

This study will use five theories of GIT to quantify the uncertainty associated with predicting and measuring damage of 8-ply composite laminate plates impacted by a projectile. We will use probability, possibility, fuzzy sets, p-boxes and Dempster-Shafer theories. Each theory will call for either single point values or intervals. Point values will be used to develop simple frequency distributions. Point values will also be used to develop possibility distributions. Intervals will be used to infer a probability distribution using Bayesian Updating. Intervals will also be used to construct Dempster-Shafer Structures and possibility distributions. Finally, a single interval [minimum, maximum] along with the data estimators (mean and standard deviation) will be used to form p-boxes. 


\section{Analysis}

The analysis consists of two portions, Uncertainty Quantification (UQ), and Fuzzy Set Interpretation. The first portion (UQ) will present an analysis in three parts: delamination area from the ultrasonic scanned test plates, calculated delamination area from finite element simulation, and finally, the combined results from the ultrasonic scanned test plates and finite element simulations. The analysis of each part will progress in complexity from a frequency analysis to the more sophisticated methods of Bayesian updating, possibility theory, p-boxes, and Dempster-Shafer theory. This will help to show the benefits of each method and their corresponding assumptions.

The second portion of the analysis shows how fuzzy sets can be used to help the subjective interpretation of damage severity and degrees of delamination. The error that occurs from calculating the area of damage as delamination is different from the error when communicating damage severity: fuzzy sets can help. We will use a Bayesian Updating scheme to justify four fuzzy sets that partition the damage continuum into overlapping and easily interpretable levels of damage. Similarly, the physical act itself of two plies separating or the act of delamination is a continuum. We will apply fuzzy sets to partition the state of delamination into three delamination states: no delamination, partial delamination, and full delamination. This can be useful in understanding model behavior for example.

\section{Uncertainty Quantification}

Frequency Analysis:

First we present a simple frequency analysis of all the data. Using a histogram, we partition the range of delamination area values into bins. Each bin will consequently contain a certain number of data points shown as a percentage of the total. We see that this is helpful to view the dispersion of data and its associated uncertainty.

\section{Bayesian Updating:}

Bayesian Updating was presented by Huyse and Thacker [14] as a way to overcome the challenge of conflicting and insufficient data which is prevalent in a wide variety of applications such as risk and damage assessment in engineering. It makes use of two key assumptions. These assumptions are important because they imply a specific initial shape to the distribution. The first assumption is the choice of Jeffrey's non-informative prior distribution. And the second assumption is the use of the Poisson distribution. The Poisson distribution essentially describes a counting process that is random in time. 
Bayesian Updating is a natural consequence of Bayes' Theorem. Bayes' Theorem simply combines prior knowledge about a parameter with additional support data to compute the subsequent knowledge of the parameter. This updated knowledge is known as the posterior distribution and is proportional to the product of the likelihood and the prior distribution as:

$$
f(x \mid X)=\frac{I(x \mid x) \cdot f(X)}{\int I(X \mid x) \cdot f(X)}
$$

Equation (IV-1) shows that the likelihood function, $I(X \mid X)$, which represents the support of $X$ given data point $x$, combines with the probability function, $f(X)$, to yield new composite knowledge $f(x \mid X)$. In our application, the likelihood (or support) of $X$ is interval data $\left[x_{1}, x_{2}\right]$ and is denoted $I(X \mid X)$ represented by equation (IV-2),

$$
I\left(X \mid\left[x_{1}, x_{2}\right]\right)=\int_{x_{1}}^{x_{2}} f(x \mid X) d x
$$

Substituting equation (IV-2) into Bayes Theorem, equation (IV-1), for interval data gives

$$
f\left(\left[x_{1}, x_{2}\right] \mid X\right)=\frac{f(X) \int_{x_{1}}^{x_{2}} f(x \mid X) d x}{\int f(X) \int_{x_{1}}^{x_{2}} f(x \mid X) d x d X}
$$

Again, two assumptions are used here in Bayesian Updating. These assumptions are the initial prior distribution and the likelihood function. First we use a non-informative prior distribution instead of a uniform prior distribution. A non-informative prior distribution attempts to represent a certain level of initial ignorance about the system [15]. Unless specific knowledge about the system is available, we feel that using a uniform distribution is erroneously applied; yet it is traditionally assigned to describe the non-informative prior distribution. Therefore, Jeffrey's non-informative prior [15] distribution over the domain $X$ (delamination area) that assumes no-observation, $x$, is used here as described by equation (IV-4)

$$
\text { Jeffrey's non-informative prior density (no observation): } \quad f(X)=\frac{1}{\sqrt{X}}
$$


Second, information (interval observations) about the structural damage area becomes the interval likelihood $I\left(X \mid\left[x_{1}, x_{2}\right]\right)$ which is a Poisson density function that updates the prior density function, Equation (IV-5).

$$
\text { Poisson density function: } f(x \mid X)=\frac{X^{x}}{x !} \exp (-X)
$$

In Equation (IV-5), $X$ represents the support (range of values) and $x$ represents a specific data point.

\section{Possibility Theory:}

Possibility distributions are developed to show the amount of nonspecificity in the data. The data that is used will be of two varieties. We will show two distributions of delamination area for each impact velocity based on the data points represented as singletons and then the data points represented as intervals. Donald [16] developed a method that we use to form the most appropriate possibility distributions using point data or interval data. It is based on the idea of consonant sets and a core interval (see Figure IV-1). Consonant sets are intervals that are progressively contained by larger intervals. We will have a small subset of intervals that are consonant. The remaining intervals will be used as evidence that is distributed in support of particular consonant intervals depending on how much intersection exists between the intervals. These intervals are first developed based on the individual data points used with a Euclidean distance measure. Next, the intervals are developed based on the lower and upper bound of results for a certain impact velocity. The resulting distributions both from the collection of individual data points and the interval lower and upper bounds will show the amount of nonspecificity and the potential bounds on the probability distributions. 


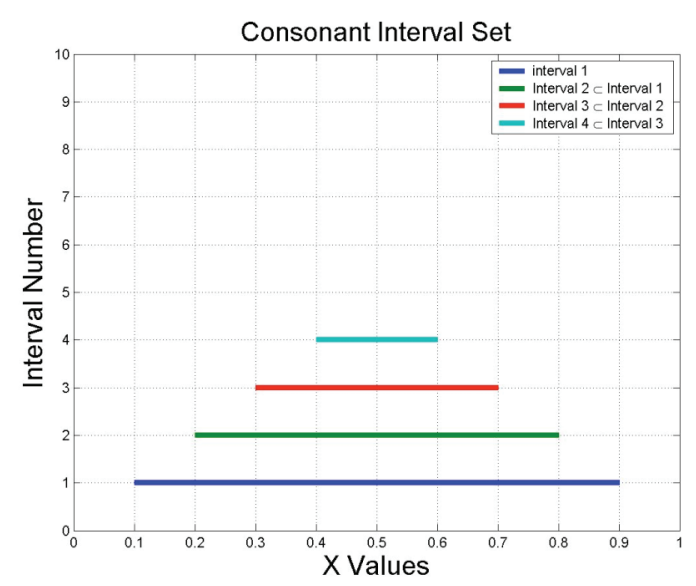

(a)

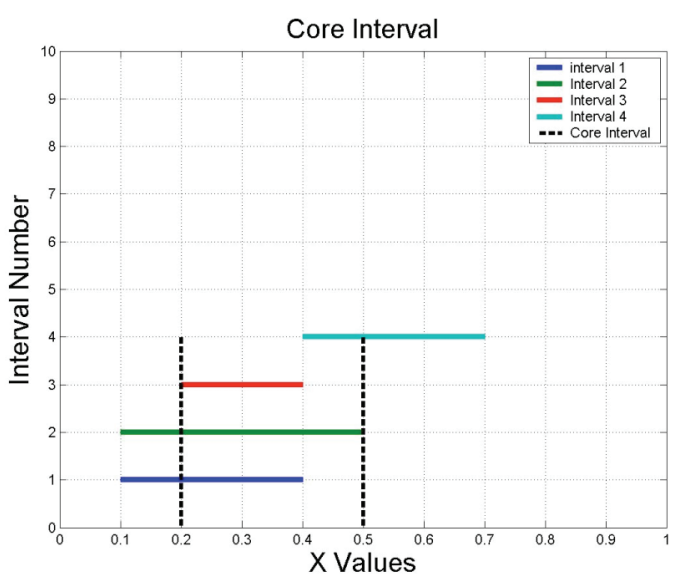

(b)

Figure IV-1. Possibility distribution concepts: (a) consonant interval set, (b) core interval.

\section{P-Boxes:}

P-Boxes (Probability Boxes) represent a more general form of probability distributions as interval bounds on probability distributions (see Ferson, et al. [17]). More specifically, p-boxes are interval bounds on cumulative distribution functions and address uncertainty in probabilities with certainty in the measurements of values. From the collection of individual data points, we determine the mean. This value along with the minimum and maximum values for the results will give the extreme bounds on the probability distribution that is consistent for that specified value of the mean. This helps to show the uncertainty inherent in selecting a single probability distribution. In order to produce the p-boxes, we use Constructor() [18]. This is a program that calculates a variety of distributions given a wide range of constraints or system knowledge. These constraints can be the known minimum and maximum values, mean, median, mode, variation, standard deviation, or the shape of the distribution and more.

\section{Dempster-Shafer:}

Dempster-Shafer structures (Figure IV-2) most commonly consist of finitely many closed intervals from the real numbers with associated precise nonnegative masses that sum to one (see Ferson, et al. [17]). In our study the intervals are delamination area. Dempster-Shafer structures are useful in situations where there is uncertainty about the measurement of values but not the probabilities. Ferson et al. [17] explain that Dempster-Shafer structures and p-boxes are related to each other. A Dempster-Shafer structure can be a discretization of a p-box. In other words, a given p-box can be approximated by discretization with a particular 
Dempster-Shafer structure. However, the converse is not true; a given Dempster-Shafer structure is only one of many Dempster-Shafer structures that correspond to a single p-box.

Dempster-Shafer structures differ from traditional probability theory mainly in two respects. First, Dempster-Shafer structures consist of focal elements, on which evidence exits, which are sets of real values and not precise points as in probability theory. Each focal element has an associated probability mass. Secondly, unlike a discrete probability distribution on the real line, where the mass is concentrated at distinct points, the focal elements of a Dempster-Shafer structure may overlap one another. Analogous to p-boxes, upper and lower bounds on the probability assignments of focal elements are established. These upper and lower bounds are called Plausibility and Belief functions, respectively. These two functions are right-continuous nondecreasing step functions from the set of real numbers into the unit interval with $n$ (where $n$ is the number of focal elements) discontinuities located at the infimum and supremum of focal elements $a_{i}$ with masses $p_{i}$. Plausibility functions represent the sum of focal element masses that overlap with the set of interest, i.e., partial support. Belief functions represent the sum of focal element masses that are contained in the set of interest, i.e., full support. Again, we use Constructor@ [18] to produce the distributions.

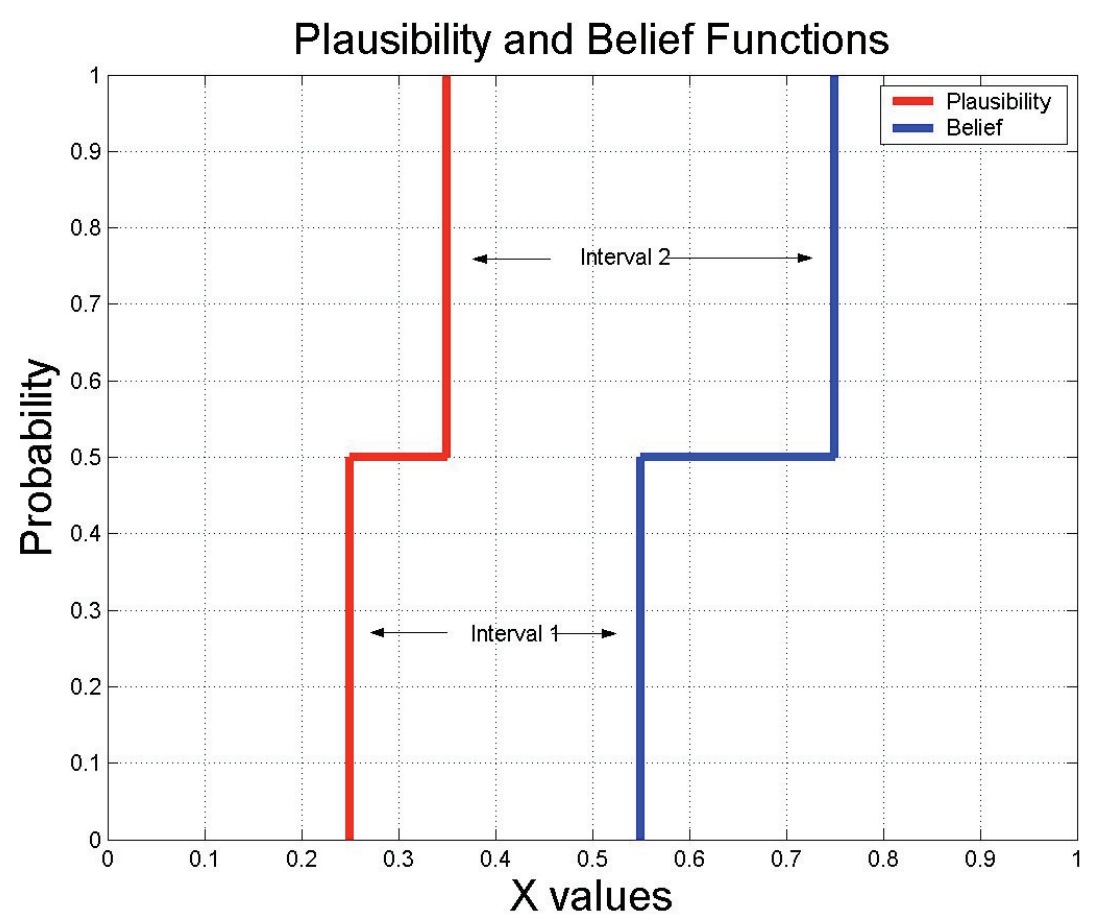

Figure IV-2. Dempster-Shafer structure consisting of two focal elements. 


\section{Ultrasonic Scanned Test Plates}

Two sets of values are used for the five plate delamination areas. Ultrasonic scans provided images of the plate delamination areas. These areas are subsequently analyzed visually using two methods in order to discern between the damage delamination areas and image irregularities. The first method relates pixels to length. Delamination area is determined by converting the pixels to length as 119 pixels/in. and then measuring the impact location's delaminated area. Furthermore, we assume a rectangular delamination area. These values are given in Table IV-B.

Table IV-B. Delamination areas and associated number of pixels.

\begin{tabular}{|c|c|c|c|c|}
\hline Identification & $\begin{array}{c}\text { Impact Velocity } \\
\mathbf{( m / s )}\end{array}$ & Width (pixels) & Length (pixels) & $\begin{array}{c}\text { Delamination } \\
\text { Area }\left(\mathbf{m m}^{\mathbf{2}} \mathbf{)}\right.\end{array}$ \\
\hline Impact L1 & 20.73 & 59 & 53 & 142.46 \\
\hline Impact L2 & 20.40 & 50 & 48 & 109.34 \\
\hline Impact M1 & 28.38 & 32 & 46 & 67.06 \\
\hline Impact H1 & 32.35 & 93 & 120 & 508.43 \\
\hline Impact H2 & 36.28 & 93 & 117 & 495.72 \\
\hline
\end{tabular}

In the second approach to determine the delamination area, we use graph paper to estimate the damage area. Similar to the previous method, the approach relates the number of boxes on graph paper to length. As such, delamination area is determined visually using the conversion, $120 \mathrm{~mm}=35$ boxes in x-direction, and $80 \mathrm{~mm}=19$ boxes in y-direction. Table IV-C shows the results of this approach.

Table IV-C. Delamination areas and associated number of boxes on graph paper.

\begin{tabular}{|c|c|c|c|c|}
\hline Identification & $\begin{array}{c}\text { Impact Velocity } \\
(\mathrm{m} / \mathrm{s})\end{array}$ & Full Boxes & Partial Boxes & $\begin{array}{c}\text { Delamination } \\
\text { Area }\left(\mathrm{mm}^{2}\right)\end{array}$ \\
\hline Impact L1 & 20.73 & 5 & 4 & 129.92 \\
\hline Impact L2 & 20.40 & 2 & 3.25 & 75.78 \\
\hline Impact M1 & 28.38 & 2 & 1 & 43.30 \\
\hline Impact H1 & 32.35 & 18 & 4 & 317.59 \\
\hline Impact H2 & 36.28 & 18 & 6.75 & 357.29 \\
\hline
\end{tabular}


Each set of delamination areas is used as point values to populate a set of observations to quantify uncertainty. However, a set of observations consisting of intervals can be derived from these sets of single point values. The values from Tables IV-B and IV-C can be combined to form lower and upper values for each impact velocity. This gives us the advantage of pooling another perspective on the information we retrieved at the same time helping to account for the uncertainty that the ultrasonic scans and our rudimentary image analysis produce. These intervals are shown in Table IV-D.

Table IV-D. Delamination area intervals for each test
\begin{tabular}{|ccc|}
\hline & Delamination Area $\left.\mathbf{~ ( m m}^{2}\right)$ \\
\hline Identification & Lower & Upper \\
\hline Impact L1 & 129.92 & 142.46 \\
\hline Impact L2 & 75.78 & 109.34 \\
\hline Impact M1 & 43.30 & 67.06 \\
\hline Impact H1 & 317.59 & 508.43 \\
\hline Impact H2 & 357.29 & 495.72 \\
\hline
\end{tabular}

The subsections that follow will outline descriptions of data used in a particular method. This outline will also include some insights that can be gleaned from the resulting distributions on delamination area. 
Frequency Analysis

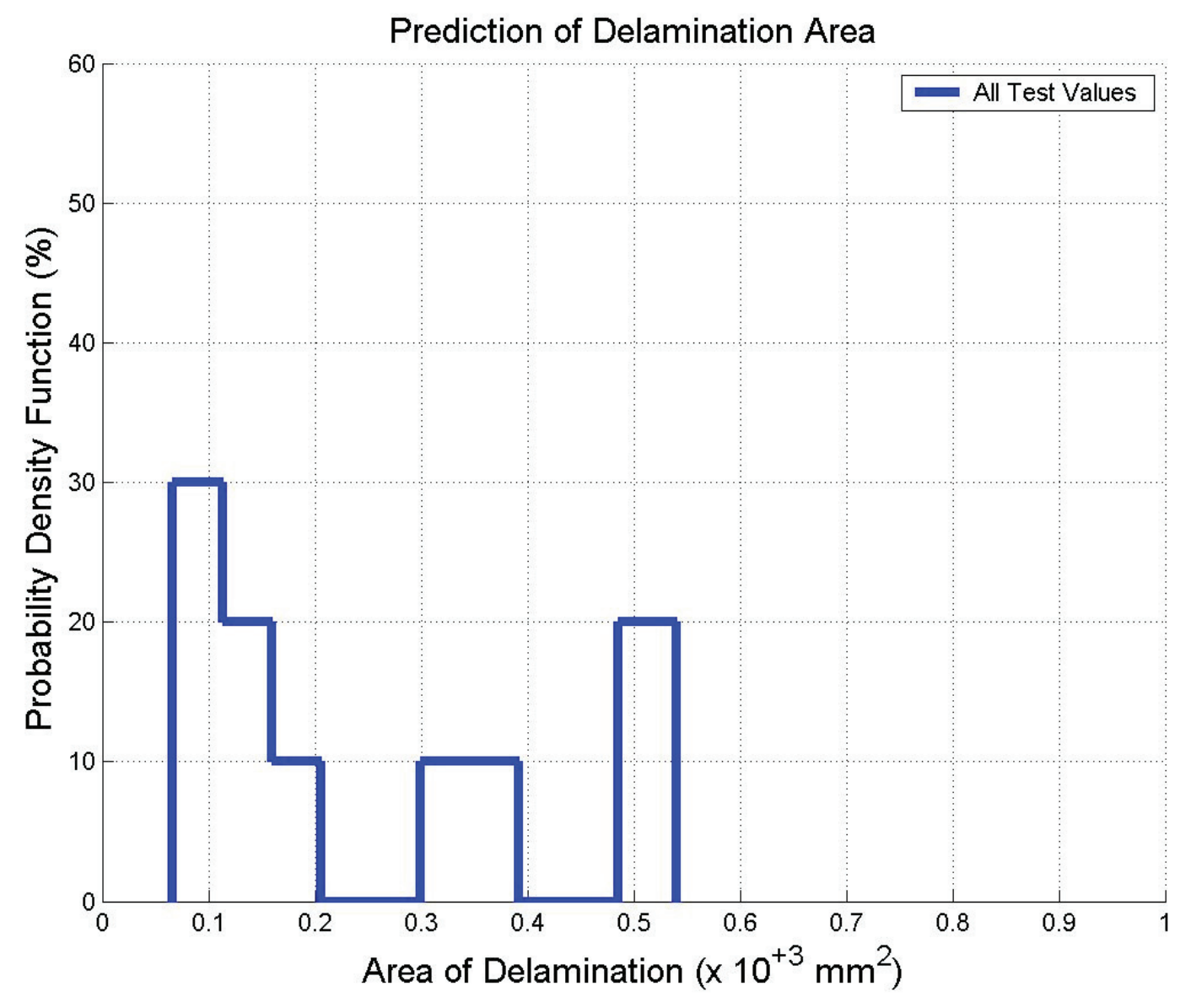

Figure IV-3. Complete data set for experimental results as a frequency distribution.

In Figure IV-3, the entire set consists of all ten data points in Table IV-D. The majority of delamination areas are the lower extreme. Specifically, $30 \%$ of the impact damage lies in the lowest range of values at around $100 \mathrm{~mm}^{2}$. Still, the entire range of impact induced damage is small, approximately $450 \mathrm{~mm}^{2}$. Additionally, the ranges (bins), [200 $\left.\mathrm{mm}^{2}-300 \mathrm{~mm}^{2}\right]$ and $\left[390 \mathrm{~mm}^{2}-480 \mathrm{~mm}^{2}\right]$ have no evidence (observations). 


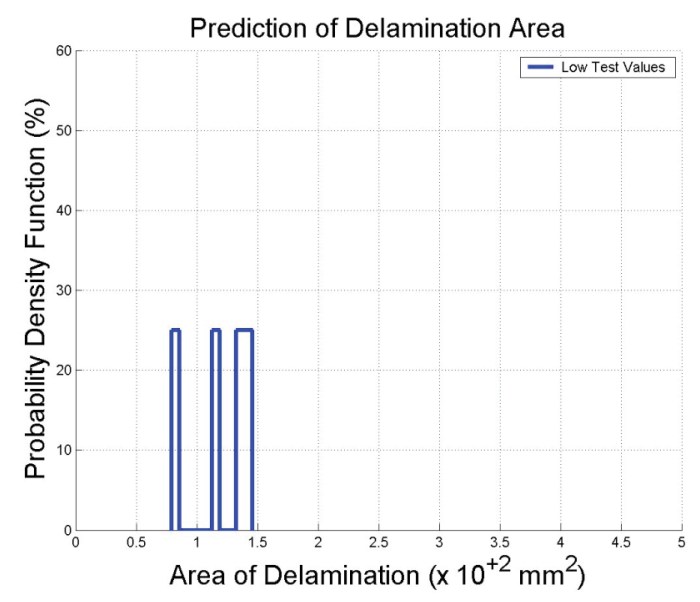

(a)

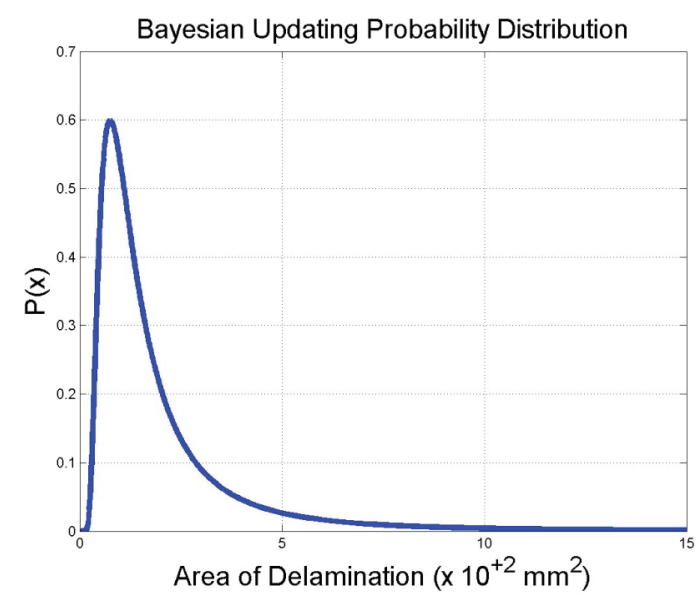

(b)

Figure IV-4. Low velocity: (a) frequency dist., (b) posterior PDF from Bayesian Updating.

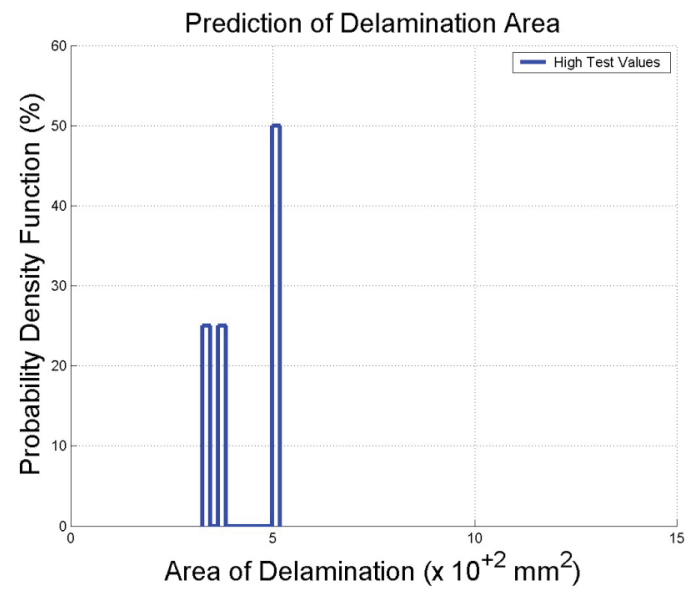

(a)

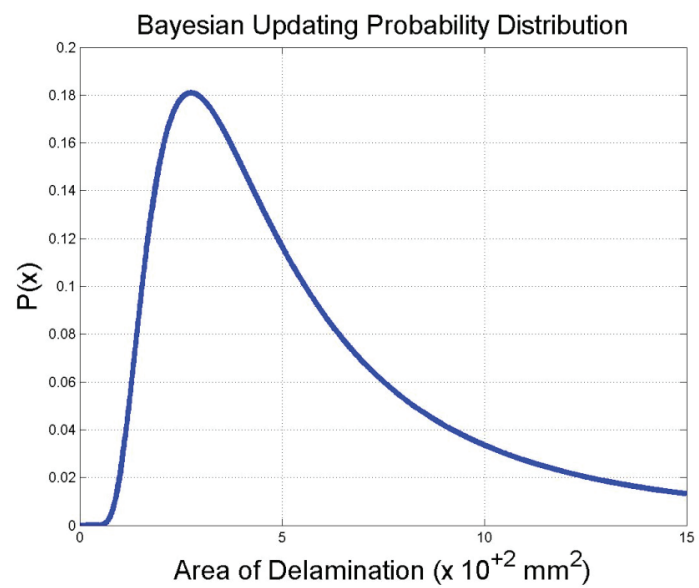

(b)

Figure IV-5. High velocity: (a) frequency dist., (b) posterior PDF from Bayesian Updating.

The data set is divided into Low and High Velocity sets. Each data set acts as likelihood when updating to form the posterior distribution as shown in Figures IV-4 and IV-5. The benefit of these posterior distributions using this approach would especially be noticed when using these values as parameters in a probabilistic approach in higher level system response. That is, in situations that require continuous distributions as parameters, the Bayesian scheme could be beneficial to develop continuity. 
Possibility:

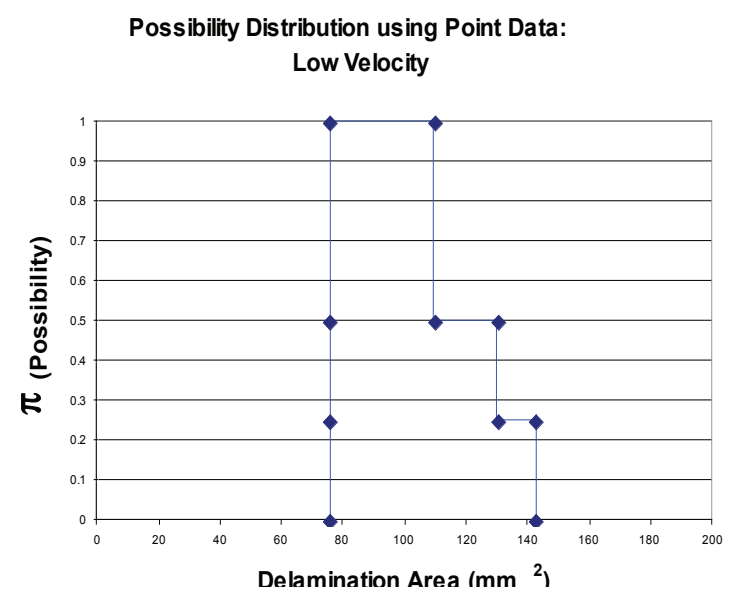

(a)

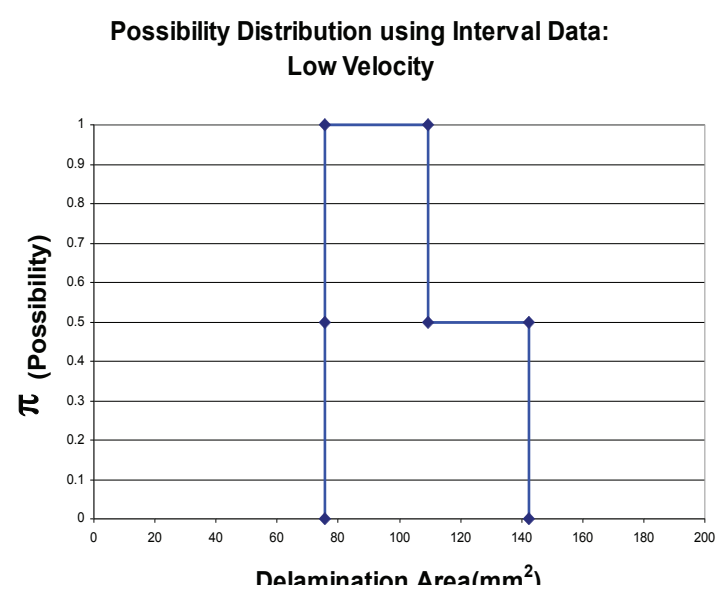

(b)

Figure IV-6. Low velocity possibility distributions: (a) from point data, (b) from interval data.

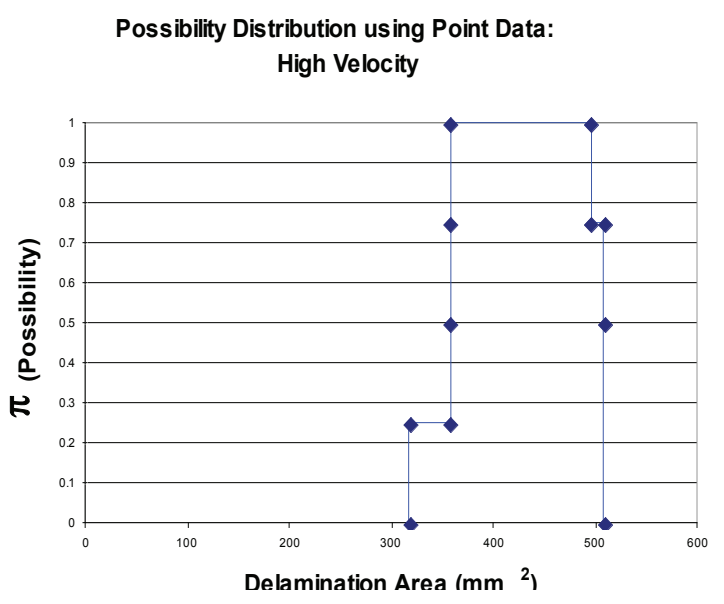

(a)

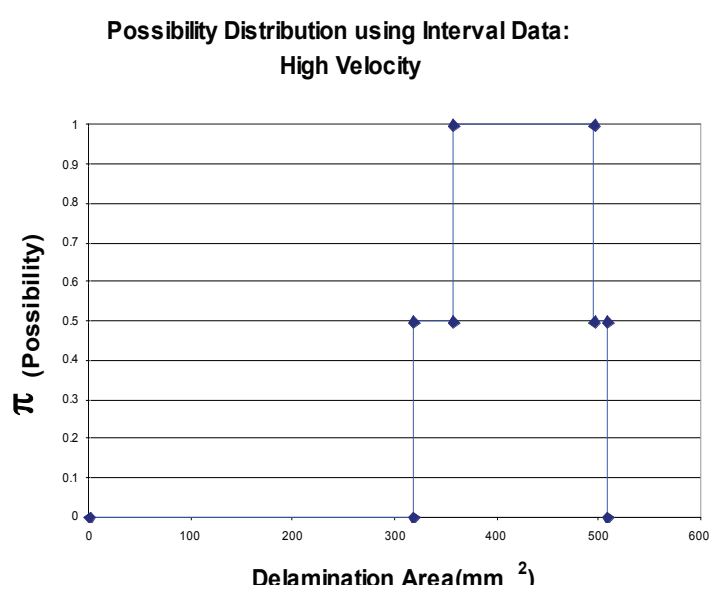

(b)

Figure IV-7. High velocity possibility distributions: (a) from point data, (b) from interval data.

The possibility distributions shown in here in Figures IV-6 and IV-7 represent the most likely or possible interval given the data at hand. Here, point data and interval data give almost identical distributions. Furthermore, both point data and interval data agree on the most possible interval. This is good in that the distribution is confirmed by two separate approaches. However, point data gives more detail than the interval data. The possibility distributions show that the small data sets yield large nonspecificity. The benefit of this approach is to see definitive threshold values for nonspecificity and a new perspective on the need for more tests. 


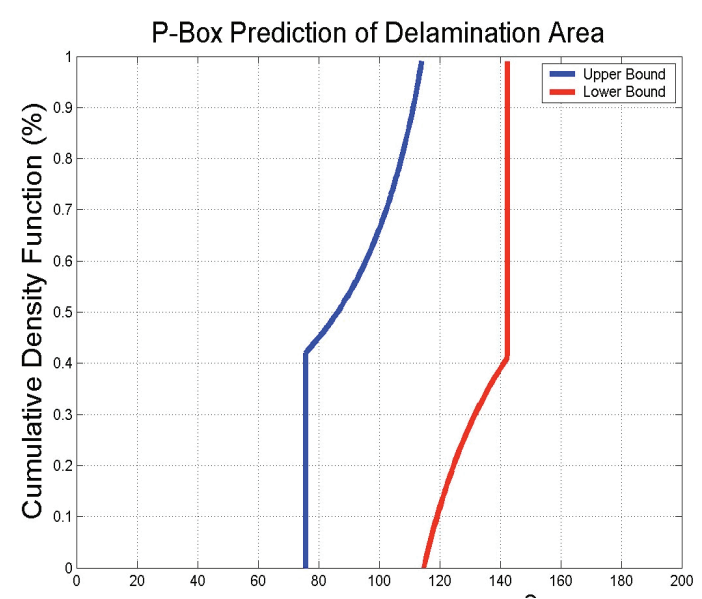

(a)

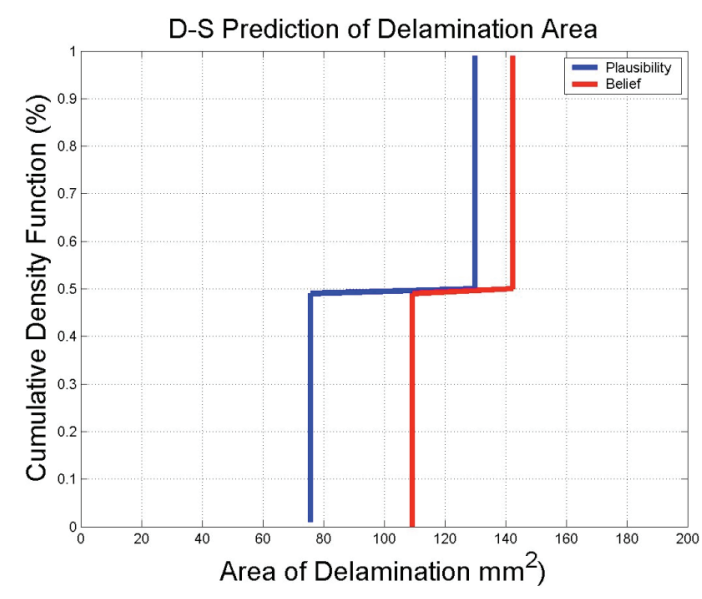

(b)

Figure IV-8. Low velocity: (a) P-box, (b) Dempster-Shafer.

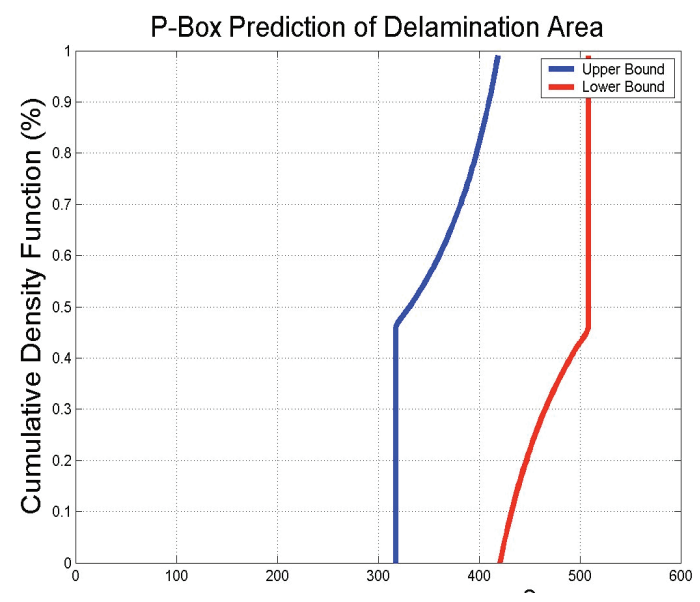

(a)

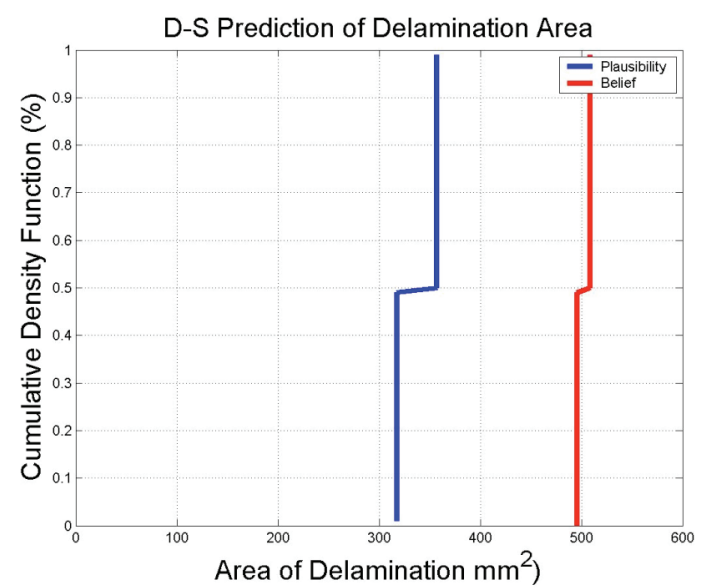

(b)

Figure IV-9. High velocity: (a) P-box, (b) Dempster-Shafer.

P-boxes and Dempster-Shafer structures are shown in Figures IV-8 and IV-9. P-box uses mean, maximum and minimum data set values. P-box represents the variation associated with randomness. All the potential probability distributions that could fit inside the CDFs would satisfy the constraints and be legitimate. Two interval focal elements in DS gives $n=2$ discontinuities. Moreover, DS gives an outer bound on p-box. The DS for the Low velocity shows no overlap. But the DS for the High velocity shows overlap. The amount of overlapping among evidence can be useful to interpret uncertainty with a particular interval (focal element). 


\section{Finite Element Simulation}

The finite element simulation consists of a parametric study totaling 24 runs where four parameters were varied: $\mathrm{Gc}, \sigma, \mathrm{k}_{\mathrm{n}}$, and projectile impact velocity. Three of the parameters, $\mathrm{Gc}$, $\sigma$, and $k_{\mathrm{nl}}$, are assigned one of two values (low or high). The fourth parameter, projectile impact velocity, is assigned one of three values (low, medium, and high). The reason for the limited choice of values is explained in Chapter III-Parametric Study. Table IV-E displays the combinatorial effect of the low, medium, and high variations. However, we are concerned primarily with the extreme values in order to more confidently encompass the uncertainty. Therefore, the impact velocities of interest are the low and high values.

This parametric study produces estimates of delamination area based on the damage function $F(\lambda)$ value of Equations (III-1) and (III-2). The predicted values of $F(\lambda)$ are presented as a contour plot. This contour plot is a visual depiction of the state of damage of the composite plate. The values of $F(\lambda)$ vary between 0 and 1 but are transformed to the range of pixel intensity values on the scale of 0 to 203. Therefore in our calculation of delamination area we need to assert a threshold pixel value below which is damage (delamination) and above which is no damage (no delamination). The threshold pixel value we chose is 203. Immediately we notice one source of uncertainty.

We don't know how to define explicitly the predicted value at which delamination occurs. Of course the extreme value $[F(\lambda)=0]$ is full delamination. But how do we calculate partial delamination? We do this by establishing a tolerance value associated with full delamination. Full delamination will yield the minimum pixel value. The tolerance value will be interpreted as a range of numbers below which there is no delamination and above which there is full delamination; in the middle of this range is partial delamination (see Chapter III, Figures III-6, III-8, and III-11). We choose this value to be Tolerance $=40$ on a scale of [0 to 203] in pixel values. Table IV-F shows the point value data associated with Tolerance $=0$ and the interval data associated with Tolerance $=40$. These values are scaled with the more accurate CZM model discussed in Chapter III. 
The results from the parametric study shown in Table IV-E were adjusted to the values in Table IV-F. The original model was updated to reflect more intuitively consistent behavior when compared with the tests as explained in Chapter III. We scale the output termed $A_{\text {scaled }}$ from run \#s 1 - 24 using the new model and its counterpart model run \#6. A reference height, $\mathrm{H}_{0}$, is calculated from an "equivalent" rectangle of the same area "A" where the CZM is only between the last two plies. Similarly, we use a reference area, $A_{0}$, based on this new model where the CZM is through the thickness of the plate, i.e. between all the plies. Both of the reference values are used to scale the remaining predicted delamination areas according to Equation (IV-6).

Table IV-E. Simulation matrix.

\begin{tabular}{|c|c|c|c|c|}
\hline Run \# & $\mathrm{Gc}\left(\mathrm{J} / \mathrm{m}^{2}\right)$ & $\sigma\left(\mathrm{N} / \mathrm{m}^{2}\right)$ & $k_{\mathrm{nl}}$ & $\begin{array}{c}\text { Velocity } \\
(\mathrm{m} / \mathrm{s})\end{array}$ \\
\hline 1 & 200 & $3.41 \mathrm{E}+07$ & 1 & 10 \\
\hline 2 & 200 & $3.41 E+07$ & 1 & 25 \\
\hline 3 & 200 & $3.41 \mathrm{E}+07$ & 1 & 40 \\
\hline 4 & 590 & $3.41 \mathrm{E}+07$ & 1 & 10 \\
\hline 5 & 590 & $3.41 E+07$ & 1 & 25 \\
\hline 6 & 590 & $3.41 E+07$ & 1 & 40 \\
\hline 7 & 200 & $4.25 \mathrm{E}+07$ & 1 & 10 \\
\hline 8 & 200 & $4.25 E+07$ & 1 & 25 \\
\hline 9 & 200 & $4.25 \mathrm{E}+07$ & 1 & 40 \\
\hline 10 & 590 & $4.25 E+07$ & 1 & 10 \\
\hline 11 & 590 & $4.25 \mathrm{E}+07$ & 1 & 25 \\
\hline 12 & 590 & $4.25 E+07$ & 1 & 40 \\
\hline 13 & 200 & $3.41 \mathrm{E}+07$ & 2 & 10 \\
\hline 14 & 200 & $3.41 \mathrm{E}+07$ & 2 & 25 \\
\hline 15 & 200 & $3.41 \mathrm{E}+07$ & 2 & 40 \\
\hline 16 & 590 & $3.41 \mathrm{E}+07$ & 2 & 10 \\
\hline 17 & 590 & $3.41 \mathrm{E}+07$ & 2 & 25 \\
\hline 18 & 590 & $3.41 \mathrm{E}+07$ & 2 & 40 \\
\hline 19 & 200 & $4.25 E+07$ & 2 & 10 \\
\hline 20 & 200 & $4.25 E+07$ & 2 & 25 \\
\hline 21 & 200 & $4.25 \mathrm{E}+07$ & 2 & 40 \\
\hline 22 & 590 & $4.25 E+07$ & 2 & 10 \\
\hline 23 & 590 & $4.25 E+07$ & 2 & 25 \\
\hline 24 & 590 & $4.25 E+07$ & 2 & 40 \\
\hline
\end{tabular}




$$
\begin{aligned}
A_{\text {scaled }} & =\left(\frac{A_{0}}{H_{0}}\right) \cdot(H) \\
& =\left(\frac{A_{0}}{H_{0}}\right) \cdot\left(\frac{A}{152.4 m m}\right)
\end{aligned}
$$

Furthermore, we obtain two sets of data: one set is based on a single threshold value made to the damage function $F(\lambda)$ as in Chapter III and the second set is based on using a tolerance value on the threshold thus yielding an interval. We realize the uncertainty that is involved in specifying a particular value for damage. This uncertainty on the damage value represents ambiguity according to Table IV-A. We believe that it is more realistic to specify a range of values that can include the incipience of damage. Damage is calculated based on the pixel value as previously described. We set a tolerance value on the full damage state to be equal to 40. This means that not only will damage be calculated based on the threshold value, but values 40 points less than this will also be calculated as damaged. Figure IV-10 shows the delamination area versus tolerance values. We see that as the tolerance increases so does the interval between the upper bound and lower bound on the damage range. We set a tolerance equal to 40 to encompass a range of uncertainty that includes a $50 \%$ variation. We believe that this value is adequate to portray the uncertainty without introducing more uncertainty. Too much uncertainty would occur at a tolerance value equal to 120 where a $200 \%$ change in the calculated delamination area would be present.

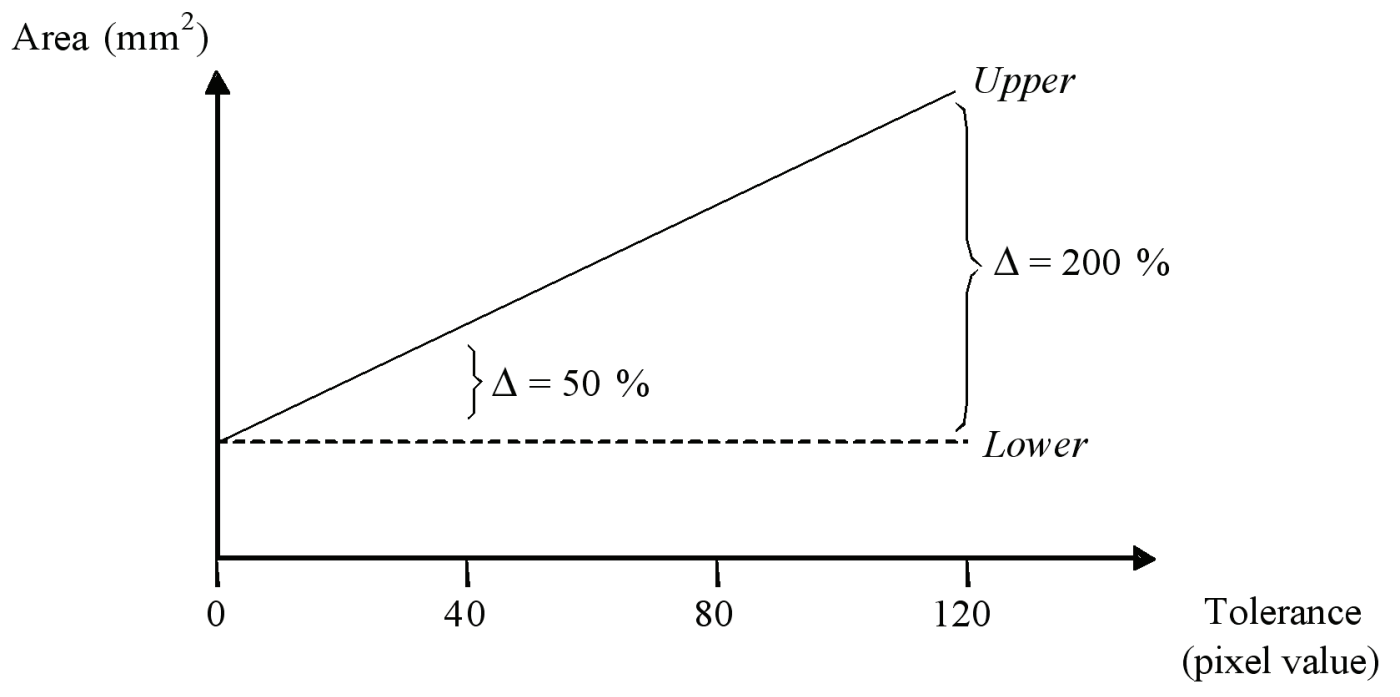

Figure IV-10. Variation of interval bounds for increasing pixel tolerance. 
Delamination areas are calculated for both low and high velocities. As previously mentioned, the low and high impact velocities in the FE simulation are $10 \mathrm{~m} / \mathrm{s}$ and $40 \mathrm{~m} / \mathrm{s}$, respectively. Table IV-F shows these delamination areas for low velocity. Notice that four of the eight simulation runs had calculations of Area $=0$, or no damage. Additionally, this table shows the individual values that are calculated using only a threshold value on the damage function. This table also shows the interval of values that were calculated using a tolerance equal to 40 points. Similarly, Table IV-G shows the delamination areas for high velocity based on the damage function for only the threshold value and the interval using tolerances of 0 and 40 , points respectively.

Table IV-F. Delamination areas for low projectile impact velocities and for Tolerance $=[0,40]$.

\begin{tabular}{|c|c|c|c|c|c|}
\hline \multirow{2}{*}{ Low Velocity Run \# } & \multirow{2}{*}{ Tolerance = } & \multicolumn{4}{|c|}{ Tolerance = 40 } \\
\cline { 3 - 6 } & Lower & Upper & Mean & STD \\
\hline 1 & 235.32 & 235.32 & 367.86 & 193.54 & 209.34 \\
\hline 7 & 353.31 & 353.31 & 491.27 & & \\
\hline 13 & 327.78 & 327.78 & 474.20 & & \\
\hline 19 & 353.68 & 353.68 & 492.38 & & \\
\hline
\end{tabular}

Table IV-G. Delamination areas for high projectile impact velocities and for Tolerance $=[0,40]$.

\begin{tabular}{|c|c|c|c|c|c|}
\hline \multirow[b]{2}{*}{ High Velocity Run \# } & \multirow[b]{2}{*}{ Tolerance $=0$} & \multicolumn{4}{|c|}{ Tolerance $=40$} \\
\hline & & Lower & Upper & Mean & STD \\
\hline 3 & 12692.16 & 12692.16 & 14081.83 & 8846.48 & 3469.43 \\
\hline 6 & 4799.94 & 4799.94 & 5326.76 & & \\
\hline 9 & 9852.49 & 9852.49 & 11171.96 & & \\
\hline 12 & 6424.41 & 6424.41 & 7098.39 & & \\
\hline 15 & 12983.95 & 12983.95 & 14386.76 & & \\
\hline 18 & 4655.45 & 4655.45 & 5207.43 & & \\
\hline 21 & 9392.61 & 9392.61 & 10634.45 & & \\
\hline 24 & 6007.79 & 6007.79 & 6827.30 & & \\
\hline
\end{tabular}


The subsections that follow will outline descriptions of data used in a particular GIT method. This outline will also include some insights that can be gleaned from the resulting distributions on delamination area.

Frequency Analysis

\section{Prediction of Delamination Area}

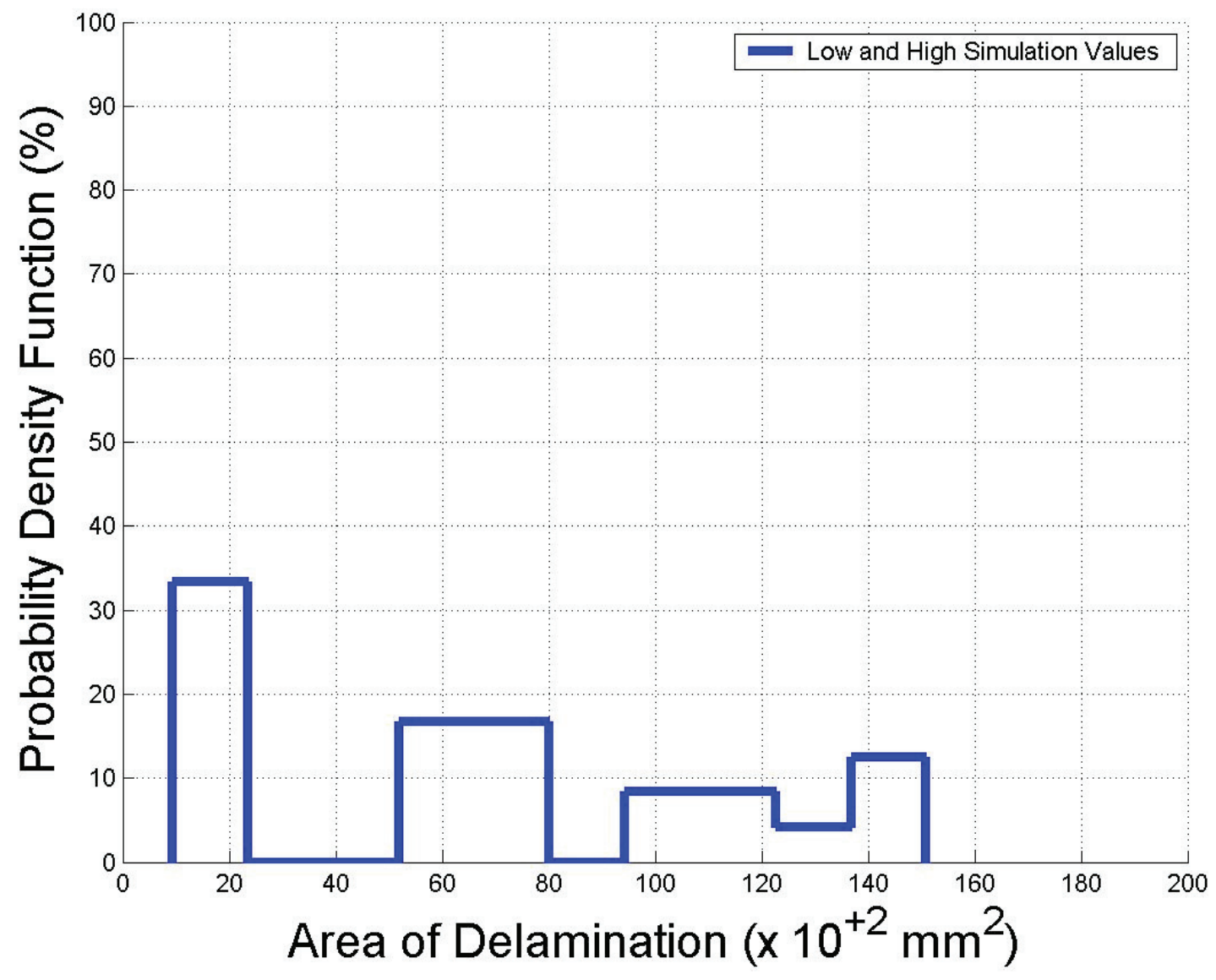

Figure IV-11. Complete data set for simulation results as a frequency distribution.

Figure IV-11 shows the distribution for the frequency analysis that consists of only the results from the Low and High impact studies from Tables IV-F and IV-G with Tolerance $=40$. The data uses the Tolerance $=40$ values. The majority of the delamination area is low. The variation in delamination area is increased for high impact velocity. As with the experimental results, we have ranges (bins) of values with no evidence, $\left[2200 \mathrm{~mm}^{2}-4600 \mathrm{~mm}^{2}\right]$ and $\left[8000 \mathrm{~mm}^{2}\right.$ $\left.9300 \mathrm{~mm}^{2}\right]$. 


\section{Bayesian Updating:}

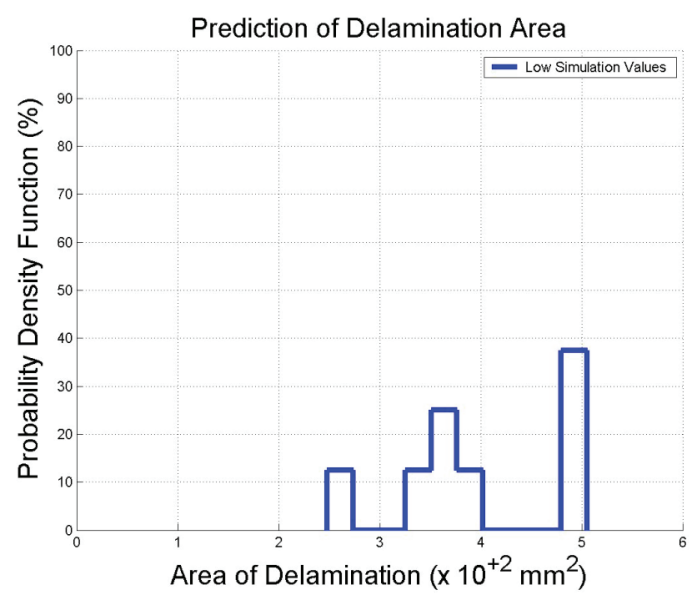

(a)

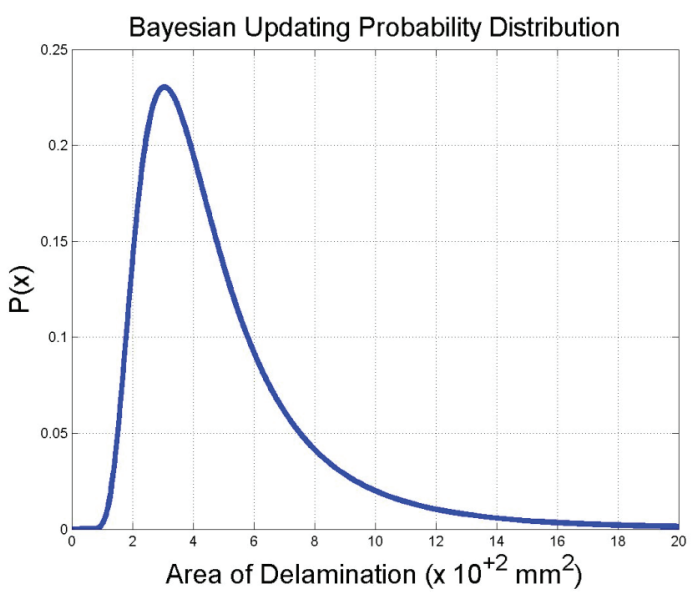

(b)

Figure IV-12. (a) Low velocity data set, (b) Bayesian Updating pdf.

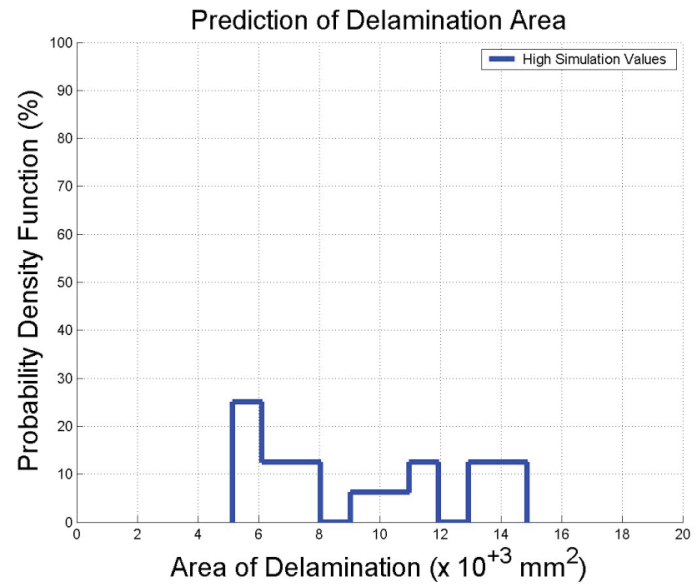

(a)

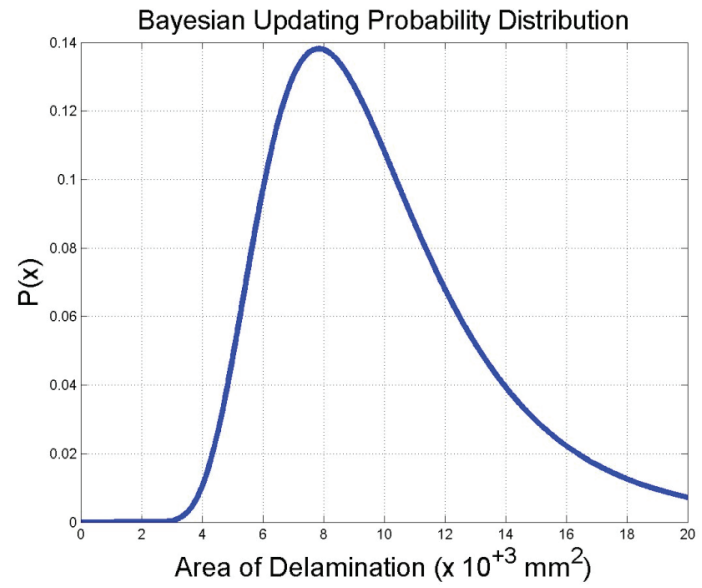

(b)

Figure IV-13. (a) High velocity data set, (b) Bayesian Updating pdf.

The data from Tables IV-F and IV-G (Tolerance = 40) are used separately to define likelihood intervals for low and high velocity posterior distributions. Figure IV-12 represents the low velocity data set and Figure IV-13 represents the high velocity data set. Posterior distributions agree with the central tendency of the data sets. It's interesting to note that the posterior distribution for the high impact velocity confirms the large variation seen in the frequency analysis. 


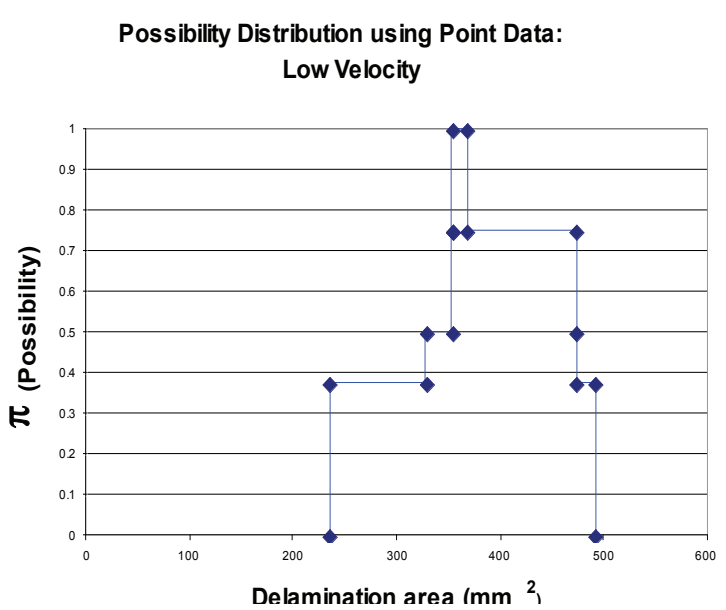

(a)

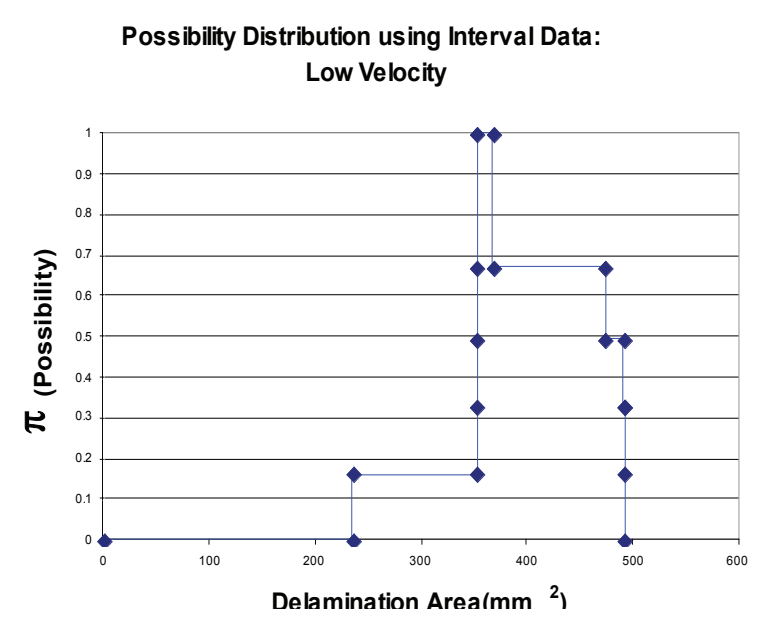

(b)

Figure IV-14. Low velocity possibility distributions: (a) from point data, (b) from interval data.

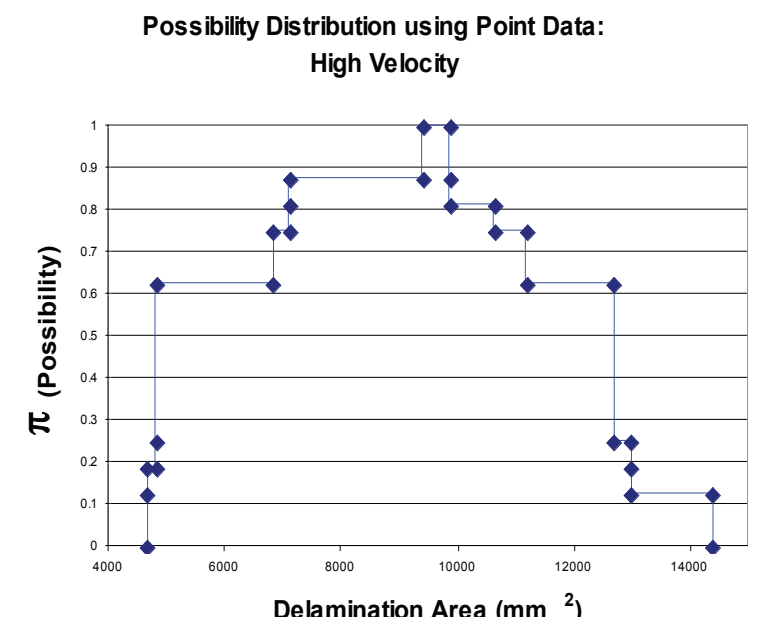

(a)

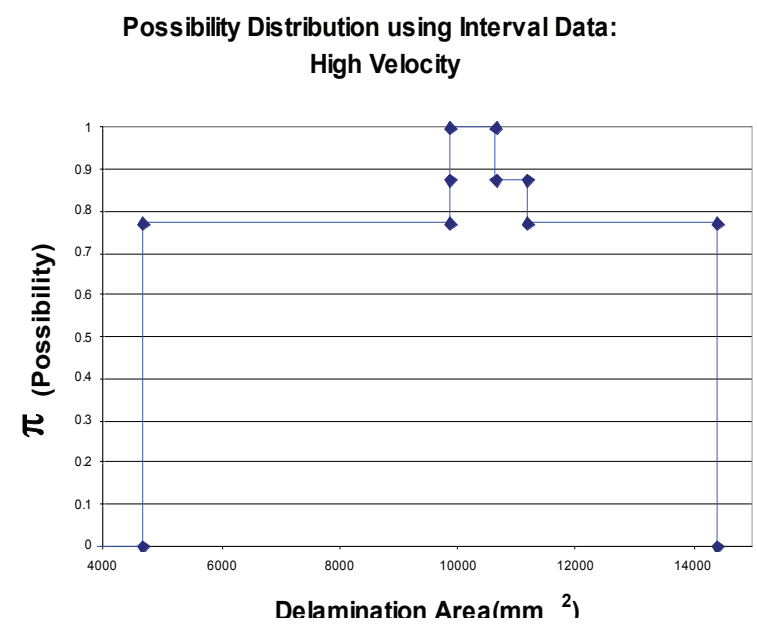

(b)

Figure IV-15. High velocity possibility distributions: (a) from point data, (b) from interval data.

The data from Tables IV-F and IV-G for Tolerance $=40$ is used as both point and interval sets to produce possibility distributions. Low velocity distributions due to point data and interval data are almost identical as seen in Figure IV-14 (a) and (b), respectively. However, the high velocity point data distribution, Figure IV-15a has a lot more detail than its interval counterpart, Figure IV-15b . Low velocity distribution cores [range where $\pi$ (possibility) $=1.0$ ] are very close. Here, we can argue that the range of nonspecificity is small enough that additional simulations are not imperative. 


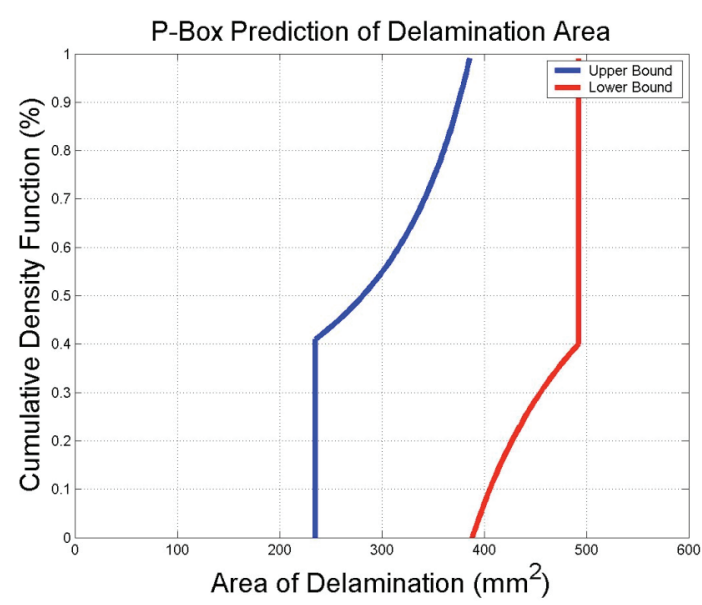

(a)

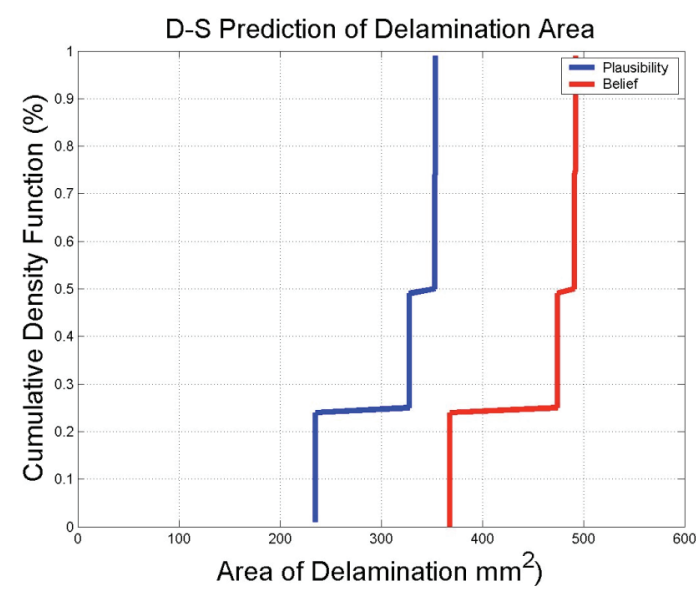

(b)

Figure IV-16. Low velocity (a) P-Box, (b) Dempster-Shafer structure.

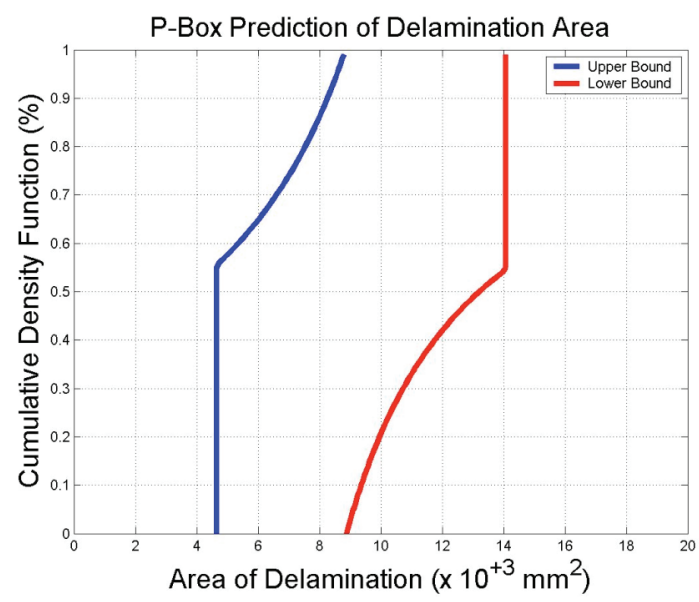

(a)

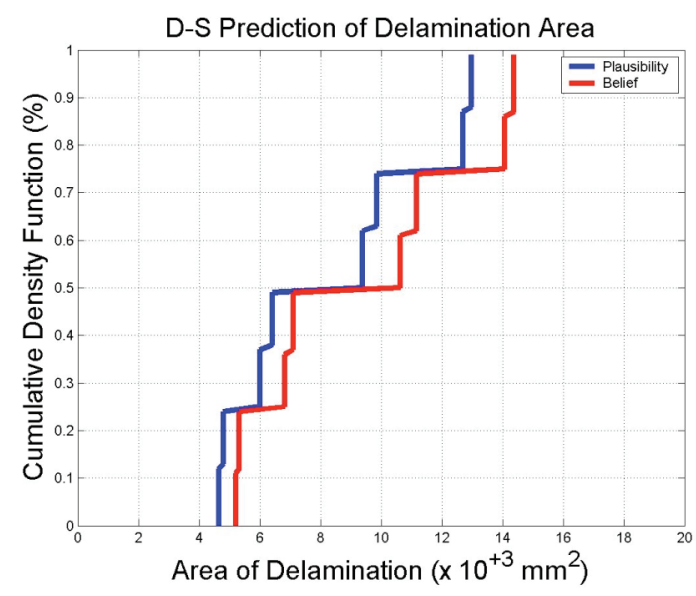

(b)

Figure IV-17. High velocity (a) P-Box, (b) Dempster-Shafer structure.

For the p-box, the mean and endpoints of the data from Tables IV-F and IV-G for Tolerance $=40$ are used as inputs to Constructor (C [18] to produce the CDF bounds (Figures IV-16a and IV-17a). For DS (Figures IV-16b and IV17-b), the intervals from Tables IV-F and IV-G for Tolerance $=40$ are used as inputs to Constructor (C) [18] to produce the Plausibility and Belief functions. Low velocity p-box shows a wide range of potential distributions. Whereas, DS for low velocity shows a much smaller range of distributions. Low velocity $p$-box is tighter than high velocity. Additionally, DS at high velocity (Figure IV-17b) shows that none of the intervals overlap. 


\section{Combined Ultrasonic Scanned Test Plates and FE Simulation}

To fully understand the scope of uncertainty regarding the prediction of delamination damage we combine the results from both studies. Figure IV-18 shows the test results for the low and high velocity projectile impact and the FE counterpart simulations as a frequency distribution. Similarly, the various theories of uncertainty as previously described are applied to the full set of data.

The subsections that follow will outline descriptions of data used in a particular GIT method. This outline will also include some insights that can be gleaned from the resulting distributions on delamination area. 


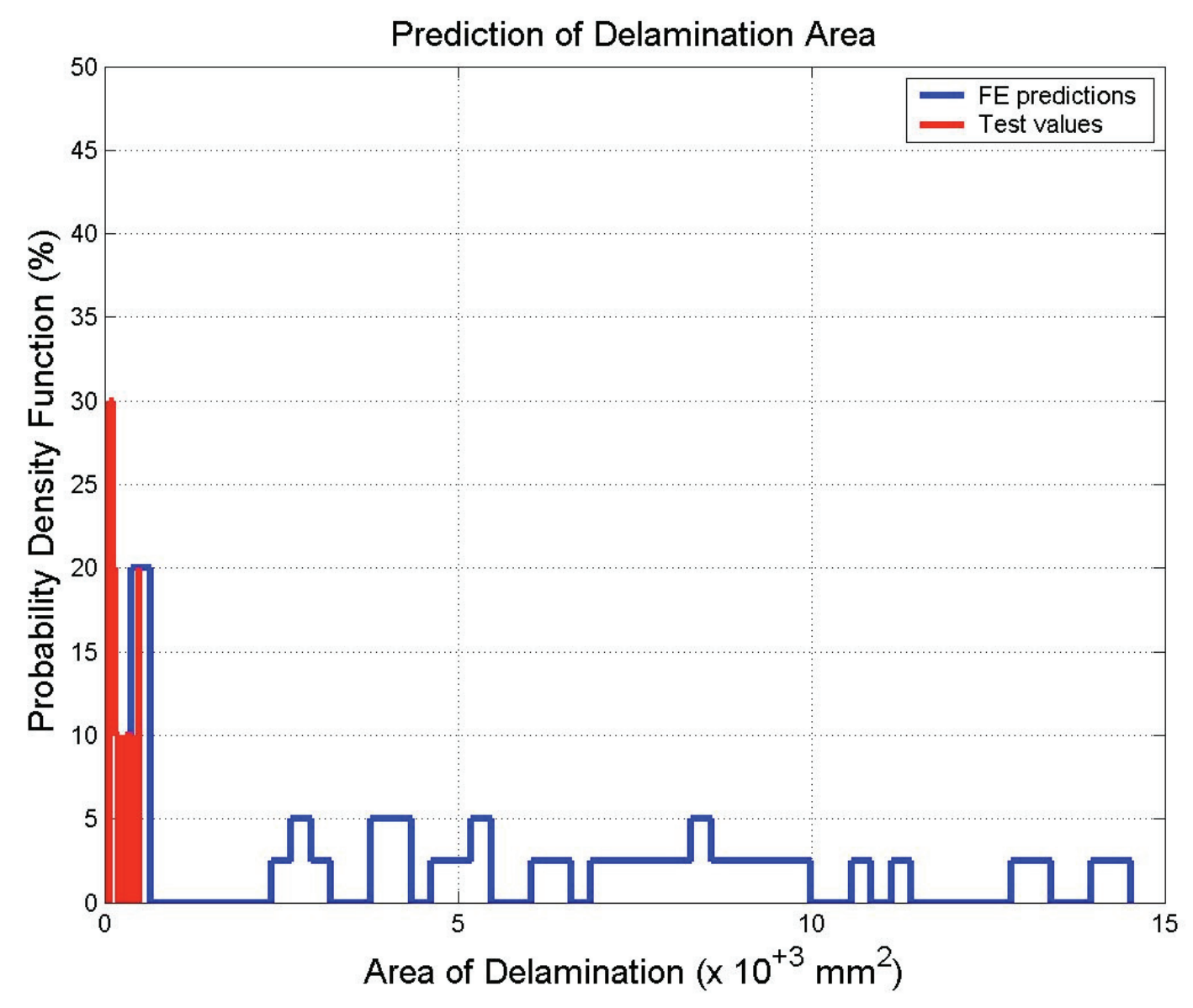

Figure IV-18. Combined plot of high impact FE predictions with corresponding test results.

Figure IV-18 shows the frequency distribution of the complete test data set, Table IV-D and Figure IV-1, combined with the data from Tables IV-F and IV-G of the simulation results plotted however into a different number of bins than in Figure IV-8 (simulation results alone). Obviously, the experimental results lie at the lowest bounds of the FE simulations. However, there is some overlap between the experimental data and FE simulations at around $100 \mathrm{~mm}^{2}$. 


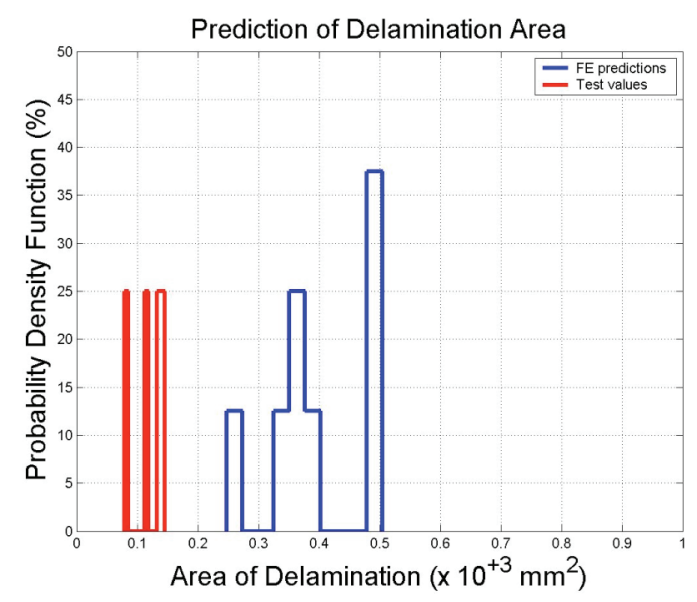

(a)

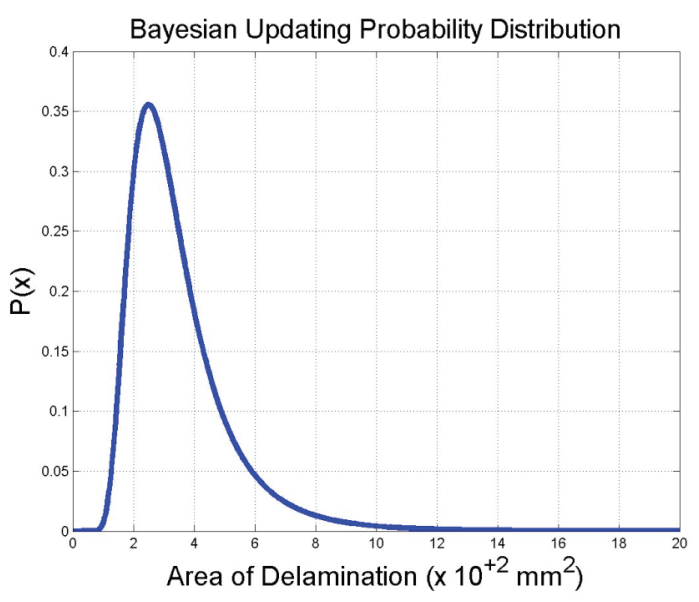

(b)

Figure IV-19. (a) Low velocity data set, (b) Bayesian Updating pdf.

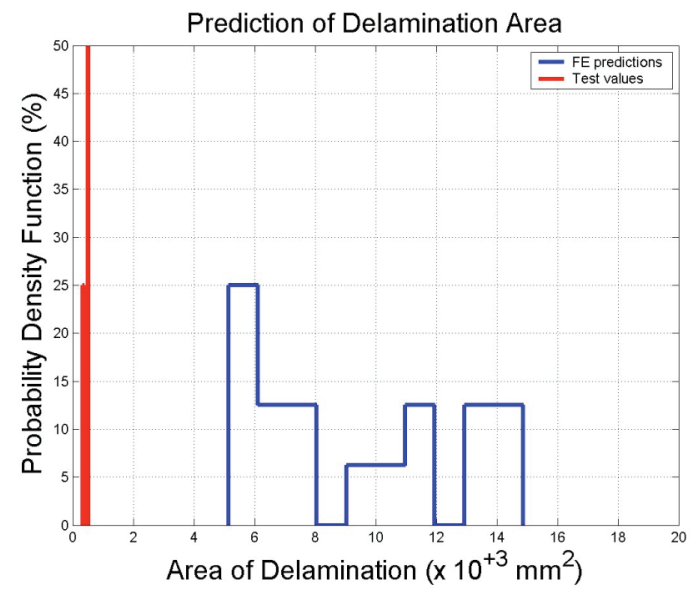

(a)

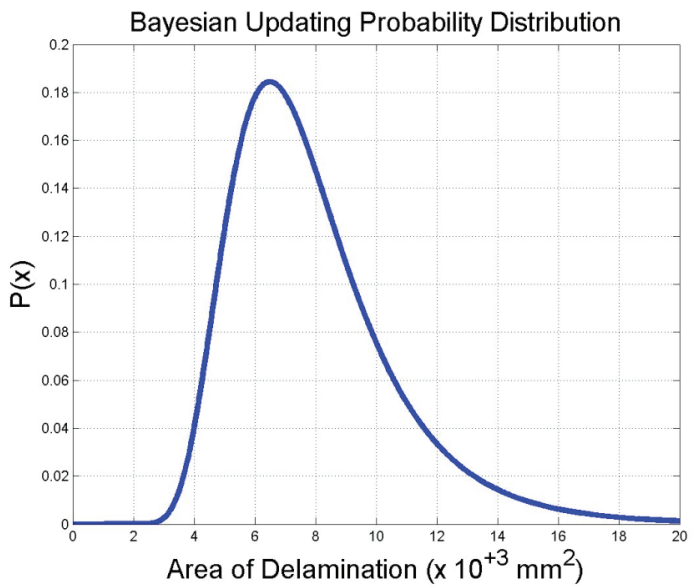

(b)

Figure IV-20. (a) High velocity data set, (b) Bayesian Updating pdf.

Like the previous cases, the complete data set used in the frequency analysis is divided into two sets: low velocity and high velocity. Both sets of values are used as likelihood when updating to get the posterior distributions: Figures IV-19 (a and b) represent the low velocity results and Figures IV-20 ( $\mathrm{a}$ and $\mathrm{b}$ ) represent the high velocity results. The posterior distribution for the low velocity accounts adequately for the data set in Figure IV-19 (a). However, the posterior distribution for the high velocity (Figure IV-20b) doesn't capture the test results adequately. Yet the influence of these test results is accounted for in the bias of probability towards the lesser values. 


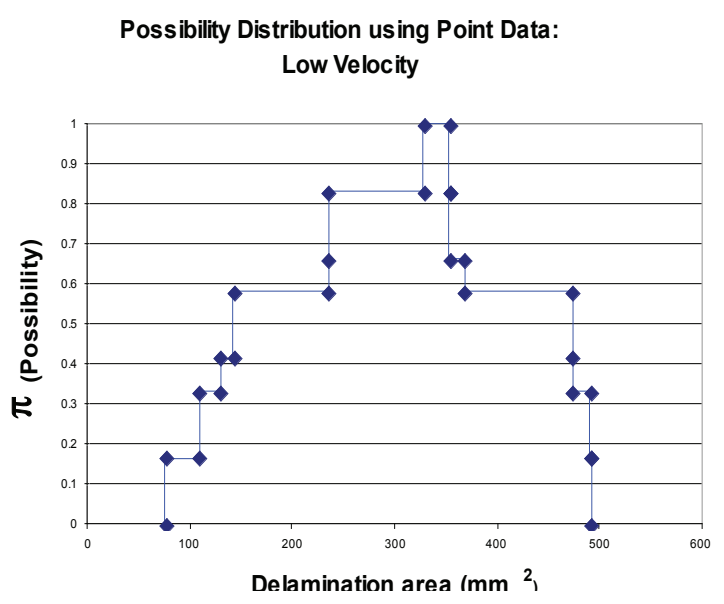

(a)

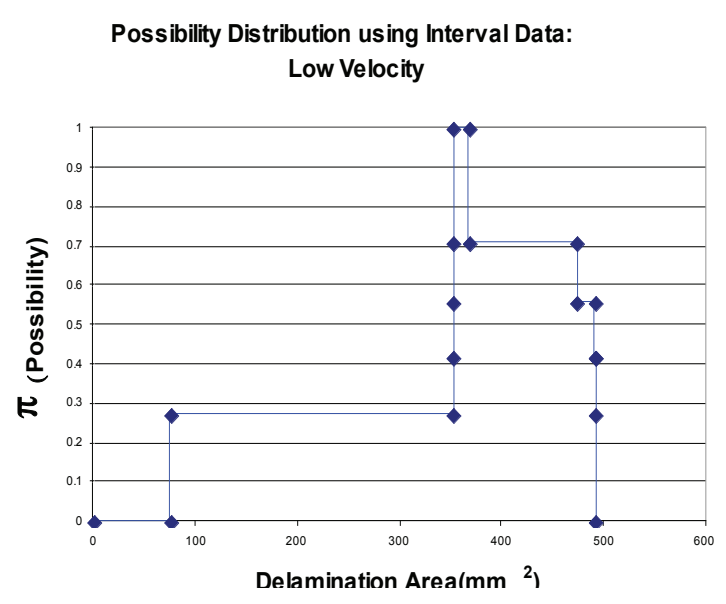

(b)

Figure IV-21. Low velocity possibility distributions: (a) from point data, (b) from interval data.

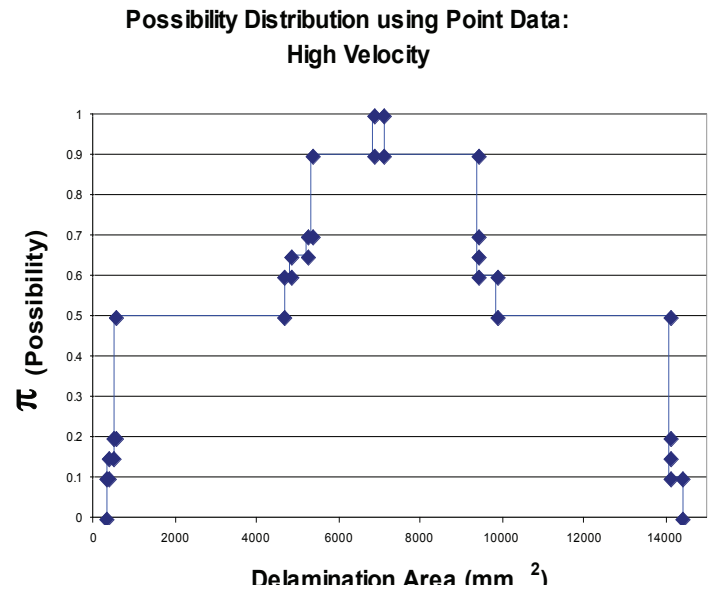

(a)

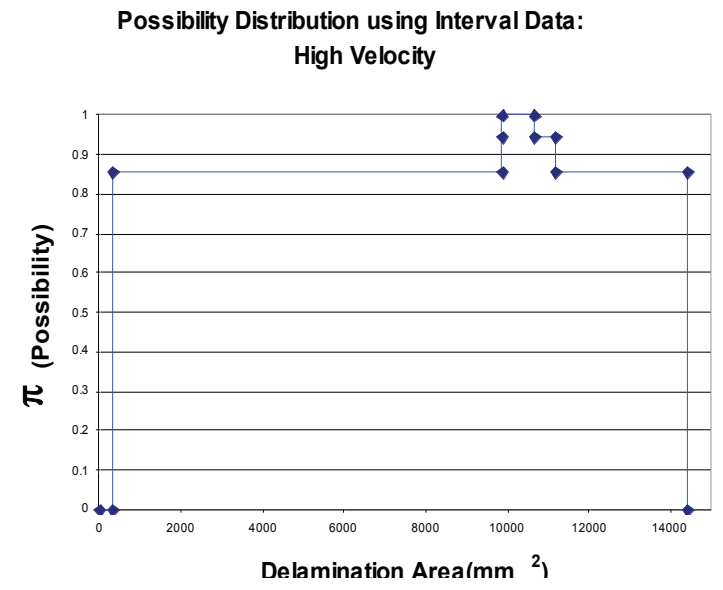

(b)

Figure IV-22. High velocity possibility distributions: (a) from point data, (b) from interval data.

Figures IV-21 ( $a$ and $b$ ) and IV-22 ( $a$ and $b$ ) represent the low and high velocity of both test and simulation data sets. The simulation values dominate the distribution. However, the most apparent influence that the test data has on the possibility distribution is seen in the support. The support expands to include the test results. Figure IV-21 (a and b) suggests more confidence in the core values since they are similar. However, Figure IV-22 (a and b) suggests that more testing is required since there is such a discrepancy in core values: the cores differ by more than approximately $2000 \mathrm{~mm}^{2}$. 


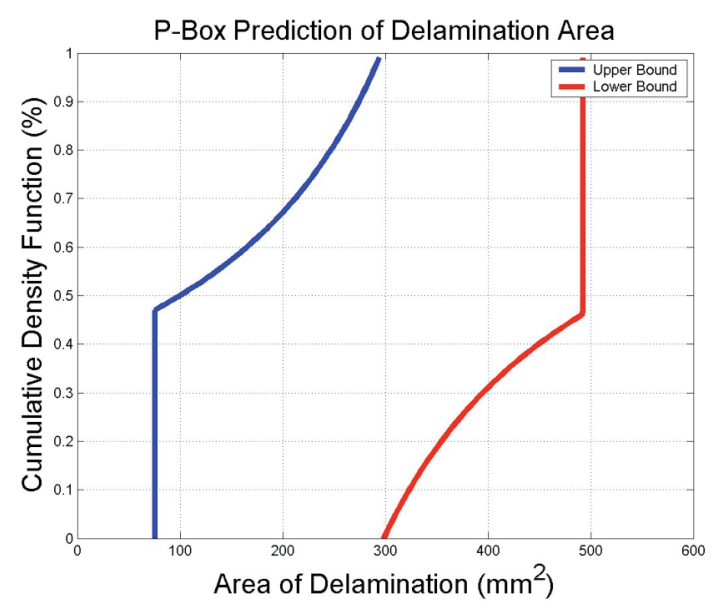

(a)

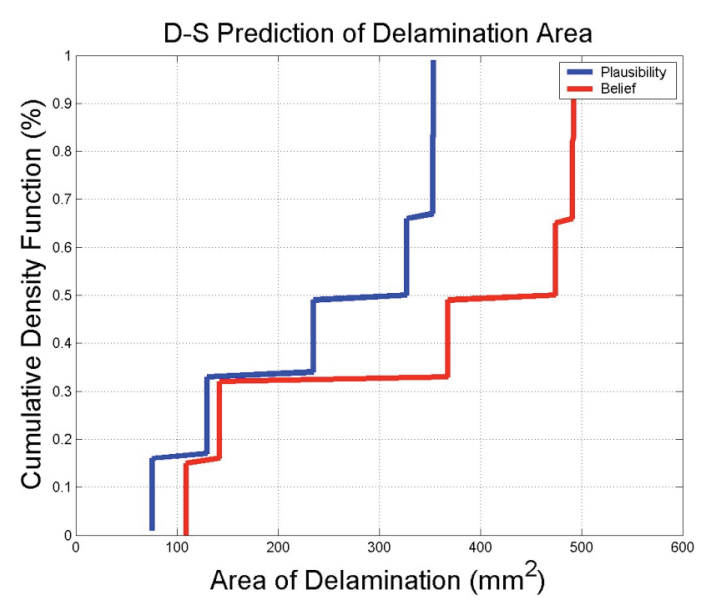

(b)

Figure IV-23. Low velocity (a) P-Box, (b) Dempster-Shafer structure.

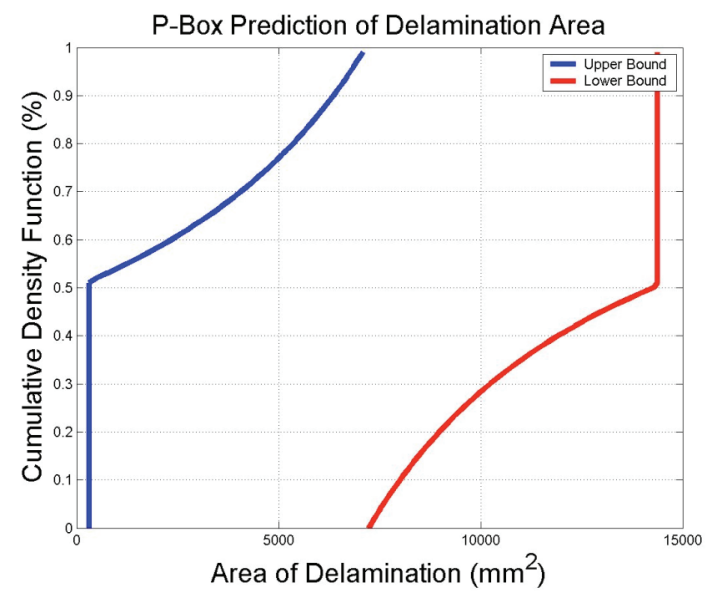

(a)

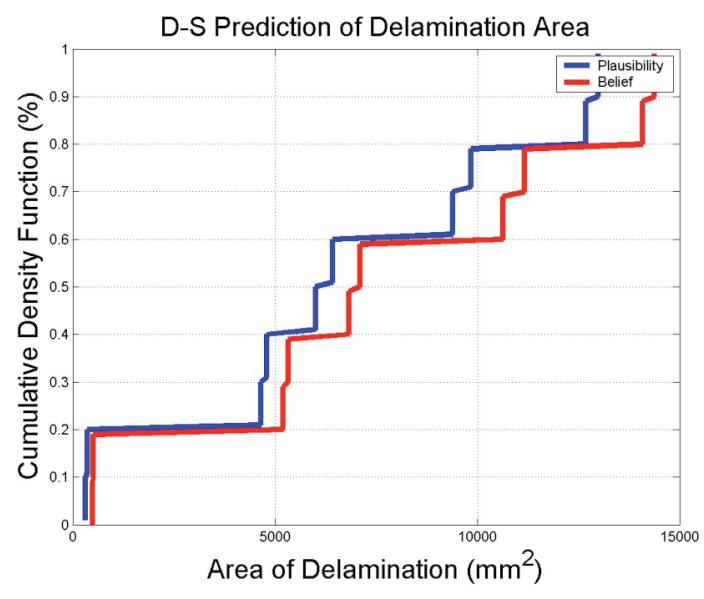

(b)

Figure IV-24. High velocity (a) P-Box, (b) Dempster-Shafer structure.

Like before, Figures IV-23 ( $a$ and $b$ ) and IV-24 ( $a$ and $b)$ use the mean and endpoint values from the test and simulation results to make the p-box but only the intervals themselves for the DS. The p-boxes show the characteristic bounding as earlier. More detail would be seen as more information is entered such as the standard deviation. The DS figures show clearly both regions of overlapping and non-overlapping intervals. 


\section{Fuzzy Set Interpretation}

Fuzzy sets are most useful for "Ambiguity" and "Interpretation" types of uncertainty. Two uses can be applied to image analysis of the data. First, we use fuzzy sets to partition the space of delamination area to be interpreted into four sets. Each of the four sets represents an ambiguous level of damage. Since the extent of damage is not a precisely defined set, we use fuzzy sets to better communicate the grading of delamination results. The second application of fuzzy sets is in interpreting pixel values that represent damage intensities. Damage intensity varies on the unit interval and does not unequivocally assert full delamination. Again, we know that delamination is a grading between no delamination and full delamination. These two applications of fuzzy sets help in a slightly different form than the uncertainty quantification in the preceding section of this report. They help to convey information and thus reduce misinterpretation.

First, we begin by assembling all the data from both experiment and simulation. Additionally, this information will include both low and high velocity results in addition to the middle velocity result from experiments. This represents the largest data set of this study. Using a Bayesian updating approach with some expert opinion, we can let the data itself "tell us" how to partition the range of delamination area values into four fuzzy sets. Figure IV-25 shows how the fuzzy sets look after this Bayesian approach is complete. We see some nice features that include the overlapping of fuzzy sets. The fuzzy sets represent the amounts of damage area, but they are given in terms of linguistic levels. These levels can be useful to communicate repair and maintenance urgency, for example. 


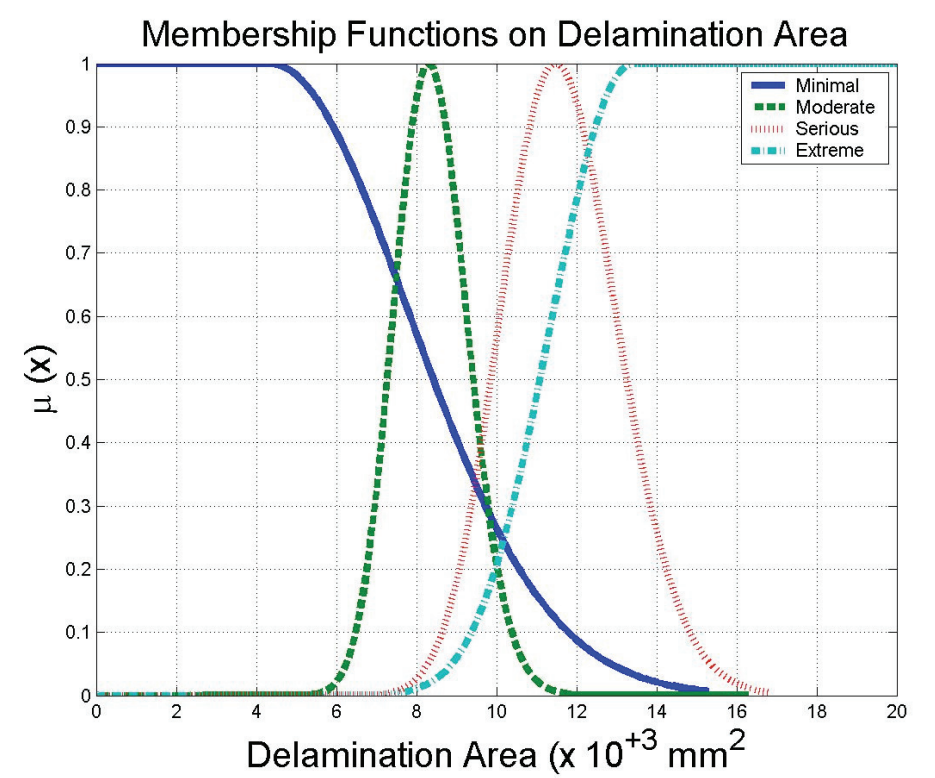

Figure IV-25. Fuzzy sets on the severity of delamination extent.

Next, we consider how to quantify values of damage yet to be interpreted as delamination. Again we let the data "tell us" how to partition the range of pixel value using a Bayesian updating approach. However, here we partition the range into three fuzzy sets: No Delamination, Partial Delamination, and Full Delamination. The partitions are simply based on the relative rate of change of delamination area as the tolerance increases. Figure IV-26 shows the three fuzzy sets and how they relate linguistic delamination levels to pixel tolerance value. On the scale of [0 - 200] where zero represents the darkest image and full delamination (or 200 for full delamination), we can infer fuzzy sets that give us an approximation to the physics of delamination. We make these inferences based on the plateaus (or regions of no change) in the areas of delamination. For example, as the tolerance increases from 0 to 20 we notice no change in delamination area; hence, the areas that we calculate as delaminated are assumed to be the most striking, given such a tight tolerance, and we qualify this as full delamination. Furthermore, as the tolerance increases beyond 20 up to a value of 60 we see a decrease in the rate of change until there is no more change. Therefore, the full delamination fuzzy set reflects this behavior giving the shape shown in Figure IV-26. Partial delamination is similarly calculated but it begins at the pixel values where the change in full delamination is noted. Partial delamination continues to a maximum and decreases symmetrically to a value of no change $(=0)$. This pixel value where there is no change is assumed to complete the fuzzy set of partial delamination. Finally, the fuzzy set of no delamination is simply the complement of the union of two sets, full delamination and partial delamination, and extends to the extreme pixel value. 


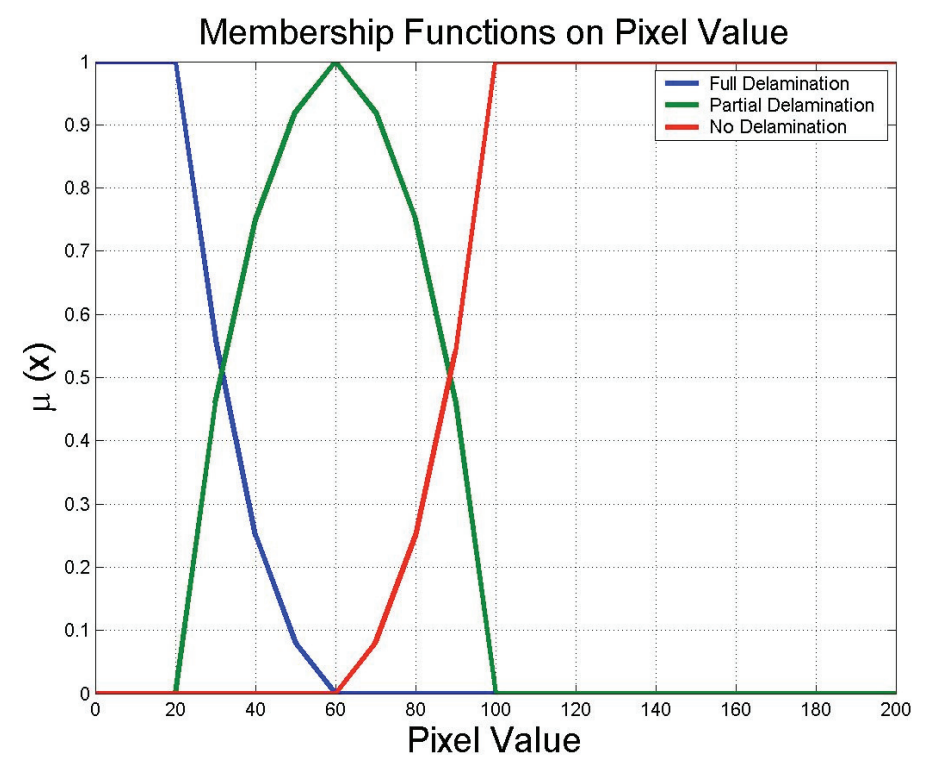

Figure IV-26. Fuzzy sets describing delamination in terms of pixel values.

\section{Summary}

We have a shown the use of five generalized information theory (GIT) methods including a probability perspective as a frequency of occurrence. Specifically, possibility, fuzzy set, p-box, and Dempster-Shafer theories were the methods illustrated in GIT. These methods gave a different perspective on the uncertainty of damage in the sense of total delamination area. The information used was either data points or intervals that came from experimental impact tests and finite element simulations. This type of analysis addresses the crux of physical system prediction that uses limited experiments in conjunction with simulation results.

The results for the $\mathrm{p}$-box and Dempster-Shafer methods were obtained using the variable synthesizing software, "Constructor (C)." Constructor (C) was beta tested in this study and proved to be very useful. It gives probability as cumulative distribution functions given a wide range of input constraints. The results were quickly produced allowing for simple analysis. This tool will become even more useful as engineers and scientists become more acquainted with it and provide more information for software development concerning their specific issues. 


\section{Conclusion}

We have investigated the uncertainty quantification of damage. This investigation has farreaching implications to fields such as structural damage prognosis. The science of structural damage prognosis is extensive and consists of many aspects. One aspect concerns how to quantify the uncertainty in damage assessment. The application upon which this investigation is made is damage to composite laminate plates subject to transverse projectile impact. We have implemented tools to address damage uncertainty in delamination area from two different sources: experimental tests and finite element simulations. Each of the two sources comes with its own uncertainties. These uncertainties can be investigated deeper within their own context. However, what we do here is to not only acknowledge that uncertainty exists but we also identify the different aspects of uncertainty for each source of evidence: test and simulation. We made progress towards the goal of quantifying each specific aspect of uncertainty by using five methods. These five methods consist of probability, possibility, fuzzy sets, probability boxes, and Dempster-Shafer structures. We are on the threshold of making significant achievements in understanding how to quantify uncertainty that emanates from different sources. This understanding can be useful for decision making in resource planning, for example.

The two sources of evidence that we used have been defined by Ferson et al. [17] as two out of five ways to obtain input evidence, "Observation of measurement," and "Modeling." The remaining three are "Direct Assumption," "Appeal to robust Bayes," and "Constraint propagation." As Ferson et al. [17] state, based on the type of input evidence, p-boxes and Dempster-Shafer structures can maintain one or all of the following properties.

- Rigor-preserving: the resultant Dempster-Shafer structure or p-box is sure to completely bound the uncertainty so long as its specification are sure bounds (sure bounds implies that the inputs are correct as opposed to guesses or sampled, random estimates),

- Best Possible: the resultant Dempster-Shafer structure or p-box could not be any tighter without more information, and

- Sample Uncertainty: the resultant Dempster-Shafer structure or p-box represents a statistical confidence claim (such as "95\% of the time the uncertain number is constructed it will completely enclose the true value or distribution"). 
Similarly, possibility theory can also address these same properties as a bound on uncertainty. Fuzzy set theory deals more with "Direct Assumption." And, in light of this type of input, we showed how to use fuzzy sets to address the issue of damage severity and delamination inception. Finally, the two probability methods that we used (frequency analysis and Bayesian updating) put a lot more assumptions on our uncertainty by implying only one probability distribution. We commented on when these methods are useful. But we must also emphasize that the two probability methods that we used presume too much knowledge about the system when we consider the type of inputs, the sources of uncertainty, and the sparseness of data. One of the dangers always present in standard probabilistic models is the need to presume specific distributions which fall outside of the information we typically have for such problems. One new procedure to get around this flaw is to use "imprecise probability theory," but this method is still under development and beyond the scope of this report.

Generalized Information Theory provides a way to address different characterizations of input evidence. We can use it to supplement probability theory when we need to understand more about the system. Additionally, GIT allows us to be more forgiving when large data sets are not available. Probability theory is well established and has proven to be very useful; yet we realize that probability theory cannot adequately represent all forms of uncertainty. We are entering a new era where we recognize that simulation and test results comprise variability, ambiguity, and conflict. This information can be synthesized to form a more encompassing assertion about the system. We are at the forefront of the development of new techniques to assess this evidence. This report can serve to illustrate how GIT can be used as a new paradigm for uncertainty quantification.

\section{References}

[1] Tippetts, T., and Hemez, F.M., "Non-Linear Models of Composite Laminates," Proceedings of the 23rd International Modal Analysis Conference, 2005.

[2] Taylor, S., Rupp, C., Johnson, D., Farrar, C., Avitabile, P., "Failure Prediction in Composite Plates with Impact-induced Damage," $22^{\text {nd }}$ SEM International Modal Analysis Conference, Dearborn, Michigan, January 26-29, 2004.

[3] Williams, T.O., and Tippetts, T., "Physics-Based Modeling of Composite Materials," Proceedings of the Materials Science and Technology Conference, 2004.

[4] Williams, T.O., "A General, Stochastic Transformation Field Theory," 2004 (in review). 
[5] Choi, H.Y., and Chang, F.K., "A Model for Predicting Damage in Graphite/Epoxy Laminated Composites Resulting from Low-Velocity Point Impact," Journal of Composite Materials, Vol. 26, No. 14, pp. 2134-2169, 1992.

[6] Chaboche, J.L., Feyel, F., and Monerie, Y., "Interface Debonding Models: A Viscous Regularization with a Limited Rate Dependency," Int. J. Solid Struct., Vol. 38, pp. 3127-3160, 2001.

[7] Tvergaard, V. "Effect of Fibre Debonding in a Whisker-reinforced Metal," Materials Science and Engineering, A125, pp. 203-213, 1990.

[8] Genz, A.C., and Malik, A.A., "Remarks on Algorithm 006: An Adaptive Algorithm for Numerical Integration over an N-Dimensional Rectangular Region," J. Comp. Appl. Math., Vol. 6, pp. 295-299, 1980.

[9] Van Dooren, P., and De Ridder, L., "An Adaptive Algorithm for Numerical Integration over an N-Dimensional Cube," J. Comp. Appl. Math., Vol 2, pp. 207-210, 1976.

[10] Tippetts, T., Beyerlein, I.J., and Williams, T.O., "New Adaptive Integration Scheme for Accurate Coarse Mesh Fracture Simulations," 2004 (in review).

[11] Nadler, B.R., Wait, J.R., Claytor, T.N., Sohn, H., and Farrar, C.R., "Effectiveness of Lamb Wave Propagation in Composite Plate Monitoring," Proceedings of the 23rd International Modal Analysis Conference, 2005.

[12] M. J. Crowe, "Pierre Duhem, the History and Philosophy of Physics, and the Teaching of Physics," Physics in Perspective, Vol. 1, 1999, pp. 54-64.

[13] Grattan-Guinness, "Karl Popper and the 'The Problem of Induction': A Fresh Look at the Logic of Testing Scientific Theories," Erkenntnis, Vol. 60, No. 1, 2004, pp. 107-120.

[14] Huyse, L., and B. H. Thacker, "A Probabilistic Treatment of Conflicting Expert Opinion," $45^{\text {th }}$ AIAA/ASME/ASCE/AHS/ASC Structures, Structural Dynamics and Materials Conference, Palm Springs, CA., 2004.

[15] Box, G., and G. Tiao, 1992 "Bayesian Inference in Statistical Analysis," John Wiley \& Sons.

[16] Donald, S., "Development of empirical possibility distributions in risk analysis," PhD dissertation, University of New Mexico, Department of Civil Engineering, Albuquerque, NM.

[17] Ferson, S., Kreinovich, V., Ginzburg, L., Myers, D., and Sentz, K., "Constructing Probability Boxes and Dempster-Shafer Structures," Sandia National Laboratories Report, SAND2002-4015, 2003.

[18] Ferson, S., J. Hajagos, D.S. Myers, and W.T. Tucker (2005). Constructor: Synthesizing Information about Uncertain Variables (http://www.ramas.com/constructor.htm). Applied Biomathematics, Setauket, New York. 
This report has been reproduced directly from the best available copy. It is available electronically on the Web (http://www.doe.gov/bridge).

Copies are available for sale to U.S. Department of Energy employees and contractors from:

Office of Scientific and Technical Information P.O. Box 62

Oak Ridge, TN 37831

(865) 576-8401

Copies are available for sale to the public from: National Technical Information Service

U.S. Department of Commerce

5285 Port Royal Road

Springfield, VA 22161

(800) 553-6847 


\section{Los Alamos}

NATIONAL LABORATORY

EST.1943 